IMPLEMENTATION OF A CONRAD PROBE ON A BOUNDARY LAYER MEASUREMENT SYSTEM

\author{
A Thesis presented to \\ The Faculty of California Polytechnic State University, \\ San Luis Obispo
}

In Partial Fulfillment of the Requirements for the Degree Master of Science in Mechanical Engineering

by

Charles Rocky Ulk

August 2010 
(c) 2010

Charles Rocky Ulk

ALL RIGHTS RESERVED 


\title{
COMMITTEE MEMBERSHIP \\ IMPLEMENTATION OF A CONRAD PROBE ON A BOUNDARY LAYER MEASUREMENT SYSTEM
}

TITLE:

AUTHOR:

DATE SUBMITTED:
Implementation of a Conrad Probe on a Boundary Layer Measurement System

Charles Rocky Ulk

August 2010

\begin{abstract}
COMMITTEE CHAIR: Russell V. Westphal, PhD, Mechanical Engineering COMMITTEE MEMBER: John Chen, PhD, Mechanical Engineering COMMITTEE MEMBER: Kim Shollenberger, PhD, Mechanical Engineering
\end{abstract}




\begin{abstract}
Implementation of a Conrad Probe on a Boundary Layer Measurement System

Charles Rocky Ulk
\end{abstract}

This thesis presents the design, calibration, and performance evaluation of a type of two-hole pressure probe anemometer known as a Conrad probe, as well as its subsequent implementation on an autonomous, compact boundary layer measurement device and its first application for subsonic in-flight measurements of a swept wing boundary layer. Calibration of the Conrad probe was accomplished using two calibration functions and a non-nulling method for resolving in-plane flow velocity direction and magnitude over a range of \pm 30 degrees. This approach to calibration and application offered the advantages of rapid data acquisition with lower energy consumption than alternative methods for pressure probe anemometry in swept wing boundary layers. Following calibration, the probe was adapted for use on an autonomous boundary layer measurement device including development of revised software. Utilizing this setup, boundary layer measurements were obtained on both swept and unswept models in a wind tunnel with a maximum operational velocity of $110 \mathrm{mph}$ corresponding to a dynamic pressure of $30 \mathrm{psf}$. The wind tunnel results showed that the Conrad probe could measure in-plane flow magnitude for both laminar and turbulent boundary layers with sufficient uncertainty and spatial resolution for its intended application in flight testing. The Conrad probe and boundary layer measurement system were then employed for flight tests of a 30 degree swept wing model carried beneath an aircraft at a flight Mach number of 0.52 and altitudes up to $44,000 \mathrm{ft}$. The flight test results from the Conrad probe allowed for the successful determination of overall boundary layer thickness, laminar/turbulent conditions, and degree of flow turning within the boundary layer. It is believed that the rapid data acquisition and low energy consumption of the Conrad probe implementation on the boundary layer measurement system make it a good alternative for future flight testing requiring measurements of inplane flow velocity magnitude and direction.

Keywords: Conrad probe, yaw, two-hole probe, calibration, non-nulling 


\section{ACKNOWLEDGMENTS}

I would like to thank Dr. Russ Westphal for his unfailing help and enthusiasm for every facet of this project, and the occasional home-cooked meal.

I would also like to thank Dr. Aaron Drake, Anne Bender, and Andrea Korntheuer for allowing the Conrad to reach 40,000 feet on their watch and the endless support they provided.

Additionally, I would like to thank Drew Hutcheson, Jonathan White, Jonathan Grubb, Tom Gwon, Stefan Laengle, and Vrajesh Patel for their invaluable support.

Support and funding from Northrop Grumman Corporation and the Air Force Research Lab are gratefully acknowledged as well. 


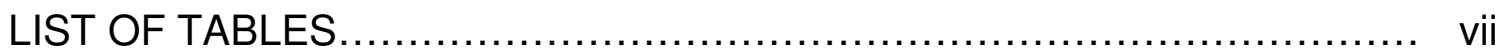

LIST OF FIGURES......................................................... vii

NOMENCLATURE........................................................ xii

\section{CHAPTERS}

1. INTRODUCTION ................................................... 1

2. PROBE DESIGN AND CALIBRATION .............................. 14

3. DATA ACQUISITION AND ANALYSIS ............................ 35

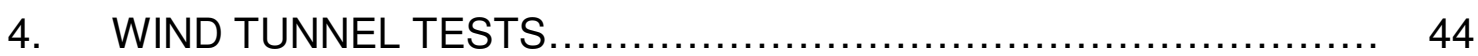

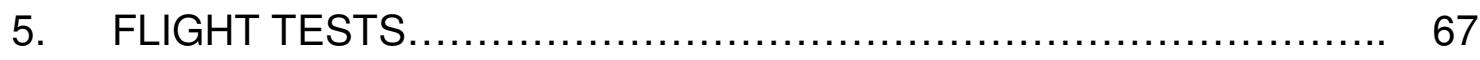

6. CONCLUSIONS .................................................... 78

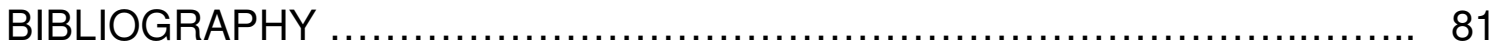
APPENDICES
A. BLDS Program Code for Conrad Probe
B. Drawings of Conrad Probe
C. Secondary Flow Calculation for Swept Flat Plate
D. Uncertainty Analysis for the Conrad Probe on the BLDS Unit 


\section{LIST OF TABLES}

Table 1.1 Comparison of probes for measurement of flow angles. 7

Table 1.2 Results from Bryer and Pankhurst for probe sensitivity versus included probe angle.

Table 1.3 Comparison of probe calibration methods from various sources.... 12

Table $3.1 \quad$ Hardware specifications for BLDS device.......................... 34

Table 4.1 Test Matrix for Wind Tunnel Evaluations.............................. 43

Table $5.1 \quad$ Test Matrix for Flight Test......................................... 67

Table C.1 Parameters for analysis of swept flat plate using Sears' Solution.... 104

Table D.1 $\quad \beta$ uncertainty results from flat plate data.......................... 108

Table D.2 $\frac{q}{q_{R E F}}$ uncertainty results from flat plate data $\ldots \ldots \ldots \ldots \ldots \ldots \ldots \ldots \ldots \ldots \ldots \ldots \ldots \ldots \ldots \ldots \ldots$ 


\section{LIST OF FIGURES}

Fig 1.1 Diagram of total (Pitot), 2-hole and 3-hole Conrad probes............ 1

Fig 1.2 BLDS unit with Conrad probe mounted for boundary layer measurements

Fig 1.3 Rotatable single-hole pressure probe with $1.3 \mathrm{~mm}, 45^{\circ}$ cut probe and $15 \mathrm{~mm}$ roll motor

Fig 1.4 Results from O. Conrad's original work for Conrad probe [doppelrohr] and claw-type yawmeter [Zweifinger Gerat] (courtesy of Technisches Messen, October 1950).

Fig 2.1 Diagram of Conrad probe......................................... 15

Fig 2.2 0.064" diameter Conrad probe.................................... 15

Fig 2.3 Tip of 0.064" diameter Conrad probe.............................. 16

Fig $2.4 \quad 0.020 "$ diameter Conrad probe..................................... 16

Fig 2.5 Tip of 0.020" diameter Conrad probe with mm scale............... 17

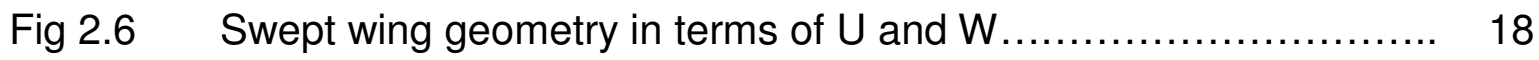

Fig 2.7 Diagram of Conrad probe and measured quantities................ 21

Fig 2.8 Conrad probe positioned for testing above $20 \mathrm{~mm}$ jet................ 22

Fig 2.9 (left) Calibration jet setup with 0.020" diameter Conrad probe (right) Calibration jet with probe on angular adjustment fixture...... 23

Fig 2.10 Diagram of calibration setup.................................. 24

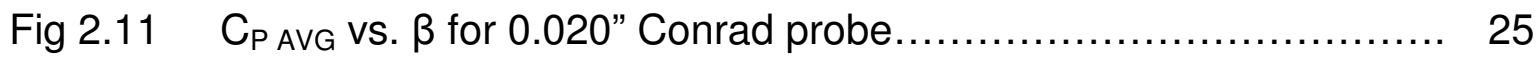

Fig 2.12 $C_{P \Delta}$ vs. $\beta$ for 0.020 " diameter Conrad probe........................ 26 


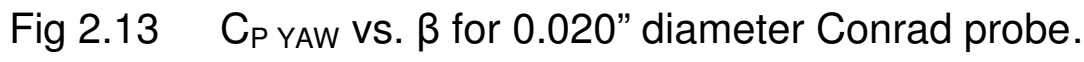

Fig 2.14 Calibration results from G.G. Brebners' work with a Conrad probe [8].

Fig 2.15 C C YAW vs. $\alpha$ for three calibration jet velocities. 30

Fig 2.16 q qe versus height (with error bars) for BLDS with 0.020" diameter Conrad probe in 2' $x 2$ ' wind tunnel on flat plate at $x \mid c=0.25$ for $\mathrm{q}_{\mathrm{REF}}=30 \mathrm{psf}$. 32

Fig 2.17 $\beta$ versus height (with error bars) for BLDS with 0.020" diameter Conrad probe in 2' $x 2$ ' wind tunnel on flat plate at $x \mid c=0.25$ for $\mathrm{q}_{\mathrm{REF}}=30 \mathrm{psf}$.

Fig 3.1 Pressure sensor and probe layout for Conrad probe configuration of BLDS unit.

Fig 3.2 Photograph of BLDS board with attached differential pressure sensor (TFX-11v2 removed)....

Fig 3.3 Collet-head L-bracket for attachment of probes to BLDS unit........

Fig 3.4 Program flow for BLDS unit

Fig 4.1 Diagram of BLDS in 2'x2' wind tunnel.

Fig 4.2 Example calculation of vertical distance "y"

Fig 4.3 BLDS unit in wind tunnel with 0.064" Conrad probe attached. 46

Fig 4.4 U $U \mathrm{u}_{\mathrm{e}}$ vs. $y$ for total and Conrad probes in 2'x2' wind tunnel on bottom surface at, 33 inches from contraction exit....

Fig 4.5 Yaw angle, $\beta$ vs. height for BLDS unit in 2'x2' wind tunnel with 0.064 " Conrad probe attached and yawed at 10.4 degrees.

Fig 4.6 2' wind tunnel test section and elliptical nose flat plate. 50 
Fig 4.7 BLDS with Conrad probe being installed on flat plate with spring load fixture.

Fig 4.8 2' wind tunnel with BLDS-C installed on flat plate.

Fig 4.9 Boundary layer profiles of velocity magnitude on elliptical nose flat plate at $\mathrm{x} / \mathrm{c}$ of 0.25 for total, rotatable, and Conrad probes.

Fig 4.10 $w \mid u_{e}$ component velocity profiles for rotatable and Conrad probes in 2'x2' wind tunnel @ x $\mid \mathrm{c}=0.25$ on elliptical nose flat plate.

Fig 4.11 Yaw angle, $\beta$ vs. height for rotatable and Conrad probes in 2'x2' wind tunnel @ $\mathrm{x} \mid \mathrm{c}=0.25$ on elliptical nose flat plate

Fig 4.12 Three-dimensional model of swept leading edge elliptical nose flat plate.

Fig 4.13 PTDS unit installed with probe foot at $x \mid \mathrm{C}=0.9$ on swept flat plate in 2'x2' wind tunnel test section.

Fig 4.14 $C_{P}$ vs. non-dimensional chord distance for swept flat plate in 2'x2' wind tunnel at $\mathrm{q}_{\mathrm{REF}}=30 \mathrm{psf}$

Fig 4.15 $\mathrm{C}_{\mathrm{f}}$ vs. non-dimensional chord distance for swept plate in 2'x2' wind tunnel at $\mathrm{q}_{\mathrm{REF}}=30 \mathrm{psf}$

Fig 4.16 Swept flat plate in 2'x2' wind tunnel with BLDS installed

Fig 4.17 Boundary layer profiles of velocity magnitude on elliptical nose swept flat plate at $x \mid c$ of 0.25 for total, rotatable, and Conrad probes.

Fig 4.18 $w \mid u_{e}$ component velocity profiles for rotatable and Conrad probes in 2'x2' wind tunnel @ x|c=0.25 on elliptical nose swept flat plate. 
Fig 4.19 Yaw angle, $\beta$ vs. height for Conrad probe in 2'x2' wind tunnel @ $x \mid c=0.25$ on elliptical nose swept flat plate.

Fig 5.1 Scaled Composites White Knight I aircraft with attached swept wing test section.

Fig 5.2 Diagram of swept wing model.

Fig 5.3 BLDS unit attached to swept wing test section on Scaled Composites' White Knight I aircraft. 68

Fig 5.4 Close-up view of BLDS unit in Conrad probe configuration attached to swept wind test section on Scaled Composites' White Knight I aircraft.

Fig 5.5 Corrected velocity profiles for total, rotatable, and Conrad probes at altitude for flights \#14, \#15, \& \#16.

Fig 5.6 (a) u|u $u_{\text {REF }} v s$. $y$ and (b) $w \mid u_{\text {REF }}$ vs. $y$ at $\beta=6.4^{\circ}$ for Conrad and rotatable probes at low altitude condition $(x \mid c=0.41$, test article upper surf.) for flights \#14 \& \#15....

Fig 5.7 (a) u|u $u_{\text {REF }} v s$. $y$ and (b) $w \mid u_{R E F} v s$. $y$ at $\beta=6.4^{\circ}$ for Conrad and rotatable probes at middle altitude condition $(\mathrm{x} \mid \mathrm{c}=0.41$, test article upper surf.) for flights \#14 \& \#15.

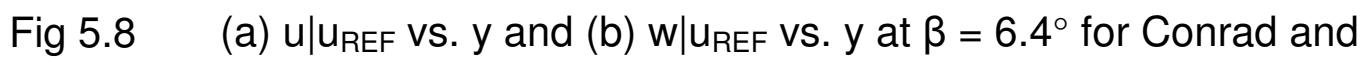
rotatable probes at low altitude condition $(x \mid c=0.41$, test article upper surf.) for flights \#14 \& \#15. 75

Fig C.1 u|U vs. height for cylinder in yawed flow 103

Fig C.2 w|W vs. height for cylinder in yawed flow. 104

Fig C.3 Maximum flow angle change versus stream-wise $(\mathrm{x})$ distance from the leading edge. 


\section{NOMENCLATURE}

\section{SYMBOLS}

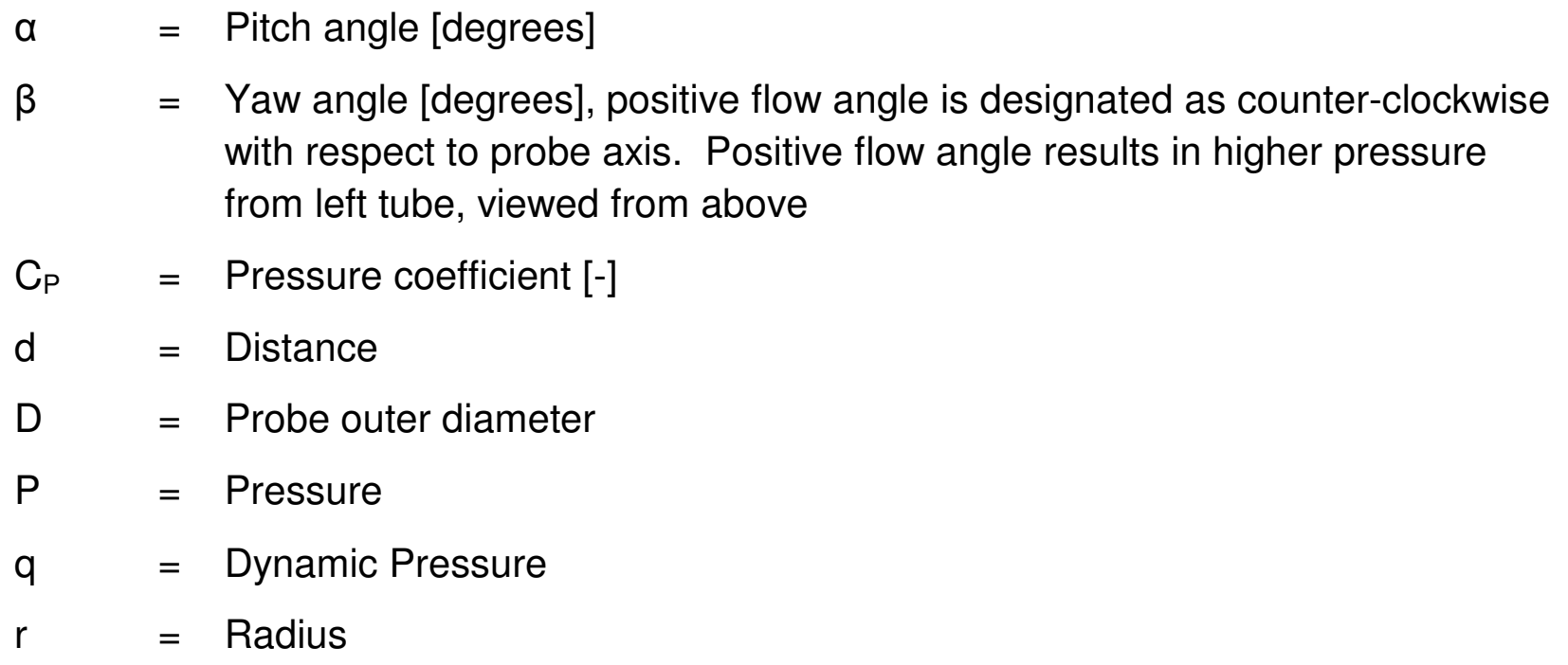

\section{SUBSCRIPTS}

$\Delta \quad=$ Pressure difference divided by average

$\mathrm{AVG}=$ Average between left and right tube measurements

$\mathrm{REF}=$ Reference measurement, generally from free-stream

$\mathrm{L}=\mathrm{L}=\mathrm{Lft}$, referring to measurements from left tube of Conrad probe

$\mathrm{R}=$ Right, referring to measurements from right tube of Conrad probe

$\mathrm{S}=$ Static, referring to static pressure

YAW = Denotes a quantity that varies with yaw angle

e $=$ Referring to a value taken at the edge of a boundary layer

le $=$ Referring to the leading edge 


\section{INTRODUCTION}

In fluid mechanics, flow measurements are carried out with the use of a variety of different types of pressure probes. The type of pressure probe used depends upon the characteristics of the flow that are required to be measured. For flows where only the velocity magnitude of the oncoming flow is needed, a simple Pitot tube or Pitot-static probe can be used. If flow direction is also needed, the complexity of the probe required increases to account for the additional angular measurements. In this case many different types of probes are able to measure the flow direction, with specific probes selected based on the angles needed to be measured in order to resolve the direction of flow. One specific type of probe used to obtain flow angle measurements, which is discussed in depth in this thesis, is known as a Conrad probe. This probe is constructed of two or three small diameter metal tubes with two of the tubes cut at an angle to make a pointed head. An example of the Conrad probe and a comparison to a Pitot probe is shown in Figure 1.1 below.

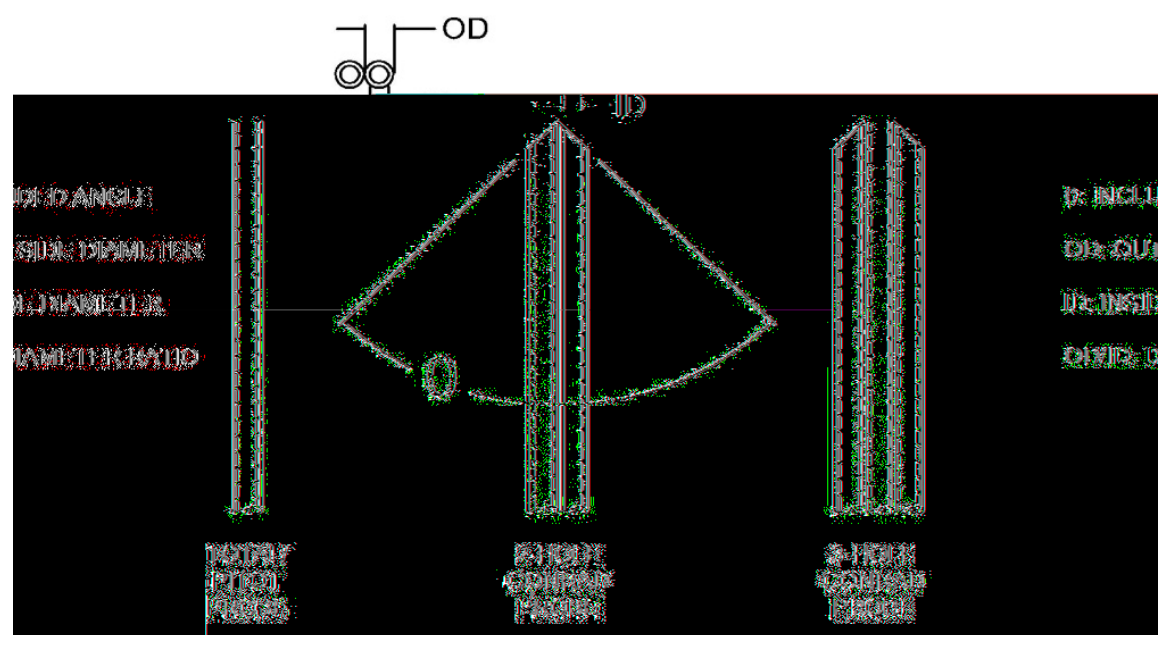

Figure 1.1 - Diagram of total (Pitot), 2-hole and 3-hole Conrad probes 
The two- and three-hole Conrad probes measure velocity magnitude and flow angle by establishing relationships between the pressures measured in the tubes. Although they can be used in any flow, the small head shape of the Conrad probe makes it ideal for measuring variations in flow velocity and angle near surfaces. These variations close to surfaces change with respect to the height above the surface and are referred to as boundary layers.

Boundary layer behavior over a surface is of key importance when designing any type of system involving the flow of a viscous fluid. In aerodynamic systems it is especially important, as boundary layer behavior determines the drag on an aircraft. Reduction of drag on aircraft systems can be achieved by designing for laminar boundary layers on the lifting surfaces of an aircraft. Design and analysis can predict laminar behavior over an airfoil to a certain extent, however even with extensive computer analysis, laminar to turbulent transitions still cannot be reliably simulated. As a result, wind tunnel measurements are often used in conjunction with computational methods. Measurement of a boundary layer over an airfoil or wing model in a wind tunnel setting is traditionally handled with the use of surface pressure sensors, some type of pressure probe or other anemometer, and a traverse system to position the probe. This approach, although useful, often fails to properly address all issues associated with design and testing of a natural laminar flow airfoil or wing. The uniqueness of the flow environment and particularly disturbances from wind tunnel to wind tunnel can negatively affect the reliability of measurements done in this way. Additionally, wind tunnel flow disturbances differ from those of in-flight 
flows causing discrepancies between laboratory measured performance and the actual performance of an airfoil or wing in the field. Model geometry can also be dissimilar to the final manufactured wing section due to real world effects such as surface finish, manufacturing tolerances, excrescences, and surface deposits on wings. Due to these issues it becomes necessary to perform in-flight boundary layer measurements to validate laminar flow wing designs. To fill this measurement niche, Northrop Grumman Corporation (NGC) has sponsored the development of devices to directly measure properties of a boundary layer in flight [1][2][3]. These devices, called the Preston Tube Data System (PTDS) and Boundary Layer Data System (BLDS), measure properties of flow on an aircraft or model surface in-flight and in such a way that the flight vehicle is not modified. The PTDS utilizes a static pressure probe, total pressure probe, and a Preston tube to measure local skin friction with the aim of determining laminar/turbulent transition location. The BLDS constitutes a more sophisticated setup, comprised of a servo motor driven stage which can step a pressure probe vertically away from a surface to obtain a boundary layer velocity profile. 


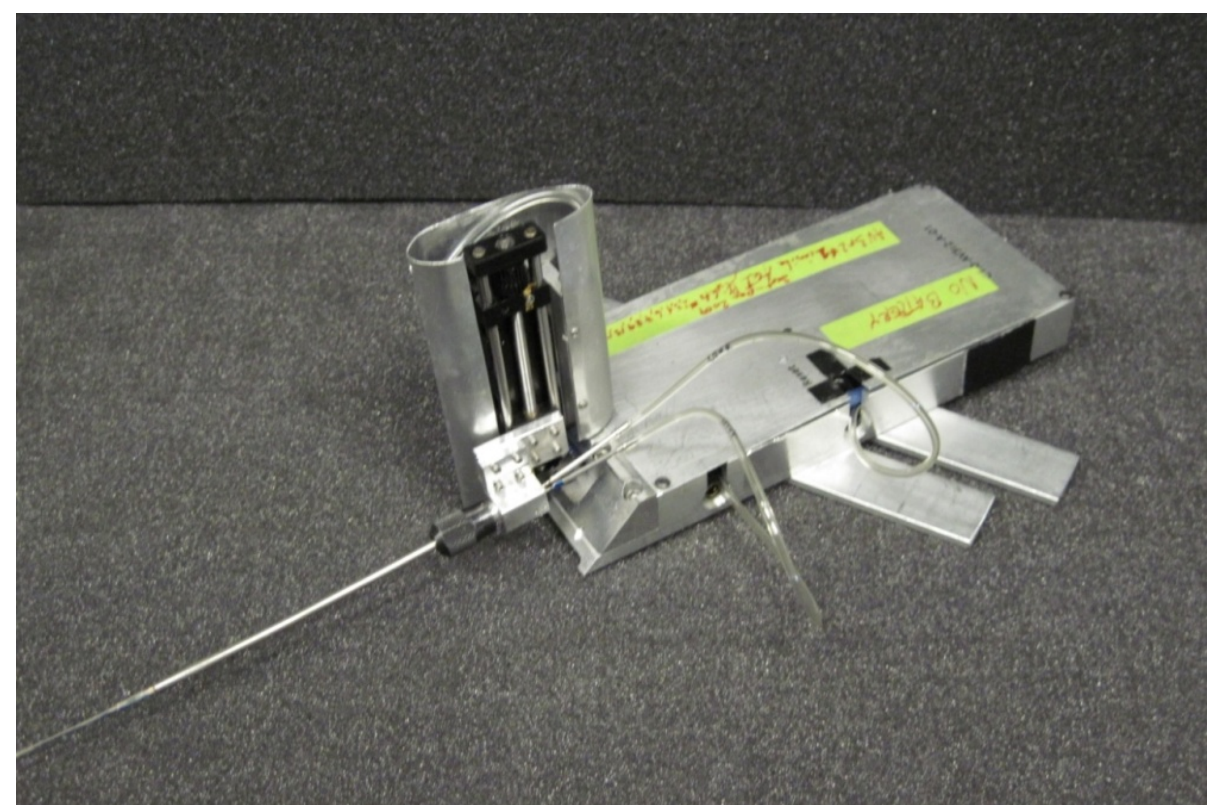

Figure 1.2 - BLDS unit with Conrad probe mounted for boundary layer measurements

The type of probe attached to this vertical stepping stage is dependent upon the type of measurement desired. On the original BLDS, a total pressure tube was used to obtain a 1-dimensional boundary layer velocity magnitude profile. With the inclusion of testing on swept wings, where flow direction changes as a function of height above the swept leading edge [4], an additional probe was needed to measure this 3-dimensional boundary layer and obtain flow velocity magnitude, along with flow pitch and yaw angles. The traditional solution to this problem is the five-hole pressure probe; however this type of probe would prove difficult to implement due to the additional pressure sensors needed to measure the pressures in each tube. To resolve this issue, a rotatable chamfered end tube was first proposed by Chu and Young [5] as a way to increase spatial resolution and reduce the number of measured pressures in applications where multi-hole probes were a hindrance. This probe consisted of 
a straight tube with its end cut at an oblique angle to its axis with a setup to rotate the tube. Further refined by Westphal et al. [6][7], a hollow shaft motor was mounted to the tube to allow for probe rotation.

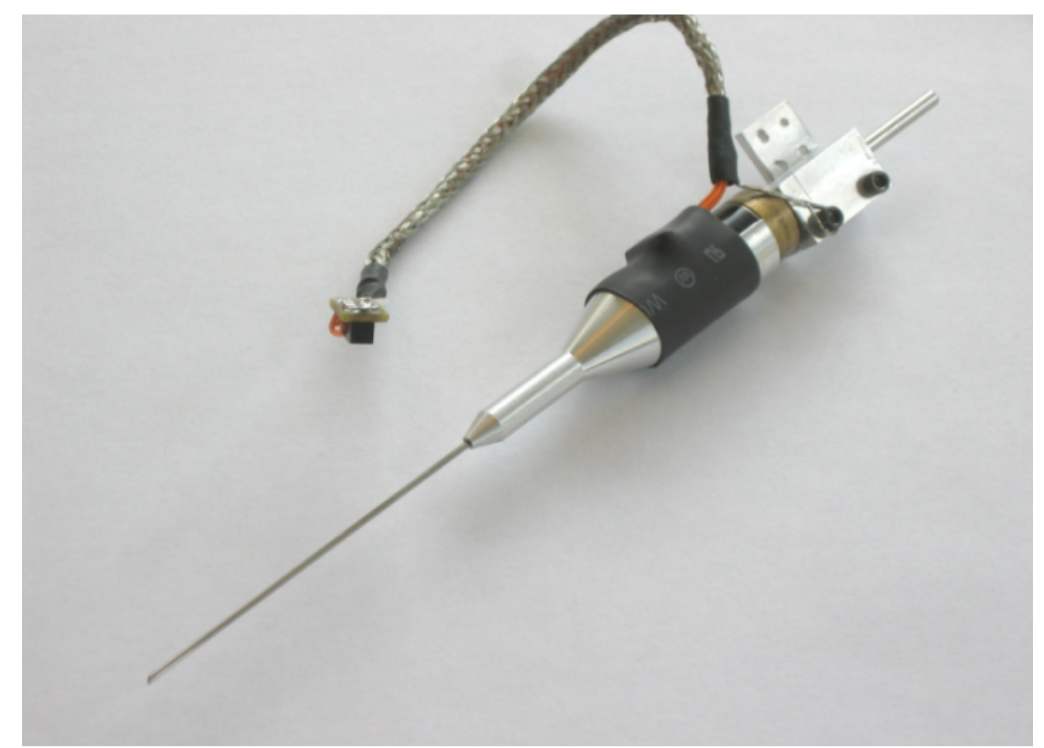

Figure 1.3 - Rotatable single-hole pressure probe with $1.3 \mathrm{~mm}, 45^{\circ}$ cut probe and $15 \mathrm{~mm}$ roll motor

The rotatable probe was found to give satisfactory results in a 3-dimensional flow, and was eventually incorporated into the BLDS and used in flight tests [8]. In many flight scenarios where measurements are carried out close to the surface, it is only necessary for the in-plane velocity components to be resolved. This reduced 2-dimensional data set presented a dilemma for the measuring capabilities of the rotatable probe due to the components that make up pitch angle no longer being needed to obtain boundary layer data. In a wind tunnel environment the redundant pitch data collected by the rotatable probe presented no problems, however in a flight test the environment is less controlled, with restrictions on device battery life due to low temperatures and on-condition test 
time due to fuel demands. These issues made the rotatable probe a less than optimal choice for taking boundary layer measurements for two reasons. The first deals with time constraints; the rotatable probe takes measurements by rotating a chamfered tube on an axis and taking six pressure sensor readings per measurement location to resolve the flow angles and flow magnitude. The additional time requirement for these readings constrains the amount of data collected in a flight test due to the finite amount of on-condition test time, device battery life, and fuel available. The second issue was due to device battery life; the BLDS device activates a multitude of different sensors to take the basic freestream, static, and temperature readings, amounting to around 20 milliampseconds per data point. Activation of additional sensors and equipment is required depending on the type of anemometer probe deployed; the rotatable probe requires activation of an additional sensor six times in addition to the energy requirement to drive the hollow shaft stepper motor, both operations requiring 40 and 100 milliamp-seconds respectively.

To employ an optimal configuration of the BLDS, a different probe was needed in order to take advantage of the simplified two-dimensional boundary layer data set, consume less energy, and increase data acquisition time. In addition to the above criteria, the probe in question had to have a small measurement volume in order to take measurements inside of a thin boundary layer. The small measurement volume made minimizing the number of tubes attached to the probe a necessity. A further comparison of probe characteristics is shown below in Table 1.1: 
Table 1.1 - Comparison of probes for measurement of flow angles

\begin{tabular}{|c|c|c|c|c|c|}
\hline & $\begin{array}{l}\text { Probe } \\
\text { Diagram }\end{array}$ & $\begin{array}{l}\text { Components } \\
\text { Measured }\end{array}$ & $\begin{array}{c}\text { Number of } \\
\text { Pressure } \\
\text { Sensors } \\
\text { Req'd } \\
\end{array}$ & $\begin{array}{l}\text { Time } \\
\text { per } \\
\text { data } \\
\text { point }\end{array}$ & $\begin{array}{c}\text { Estimated Energy } \\
\text { Consumption } \\
\text { (mA-s per data pt, } \\
1 \text { sec. avg) }\end{array}$ \\
\hline $\begin{array}{c}\text { Rotatable } \\
\text { Probe }\end{array}$ & & $\begin{array}{c}\text { Velocity } \\
\text { Magnitude } \\
\text { Yaw Angle } \\
\text { Pitch Angle }\end{array}$ & 1 & $6 x$ & 170 \\
\hline $\begin{array}{c}\text { Five-hole } \\
\text { probe }\end{array}$ & & $\begin{array}{c}\text { Velocity } \\
\text { Magnitude } \\
\text { Yaw Angle } \\
\text { Pitch Angle }\end{array}$ & 5 & $1 x$ & 60 \\
\hline $\begin{array}{c}\text { Two-hole } \\
\text { Conrad } \\
\text { Probe }\end{array}$ & & $\begin{array}{l}\text { Velocity } \\
\text { Magnitude } \\
\text { Yaw Angle }\end{array}$ & 2 & $1 x$ & 40 \\
\hline
\end{tabular}

Based on the criteria, the two-hole Conrad probe was found to be an acceptable substitute for the rotatable probe in measuring velocity magnitude and flow direction in the boundary layer on the BLDS due to its low energy consumption, small head size, and fast data acquisition time.

Conrad probes of the two and three tube varieties have traditionally been used in fluid mechanics to experimentally determine the incidence angle of flow in the plane of the tubes. This is accomplished by measuring the pressure difference 
between the tubes of the Conrad probe. The first application of this was realized by O. Conrad in 1950 [9] with his "doppelrohr". The doppelrohr consisted of two small soldered Pitot tubes joined together along their length with ends cut at angles to form a symmetric "V" shape as shown in Figure 1.4.

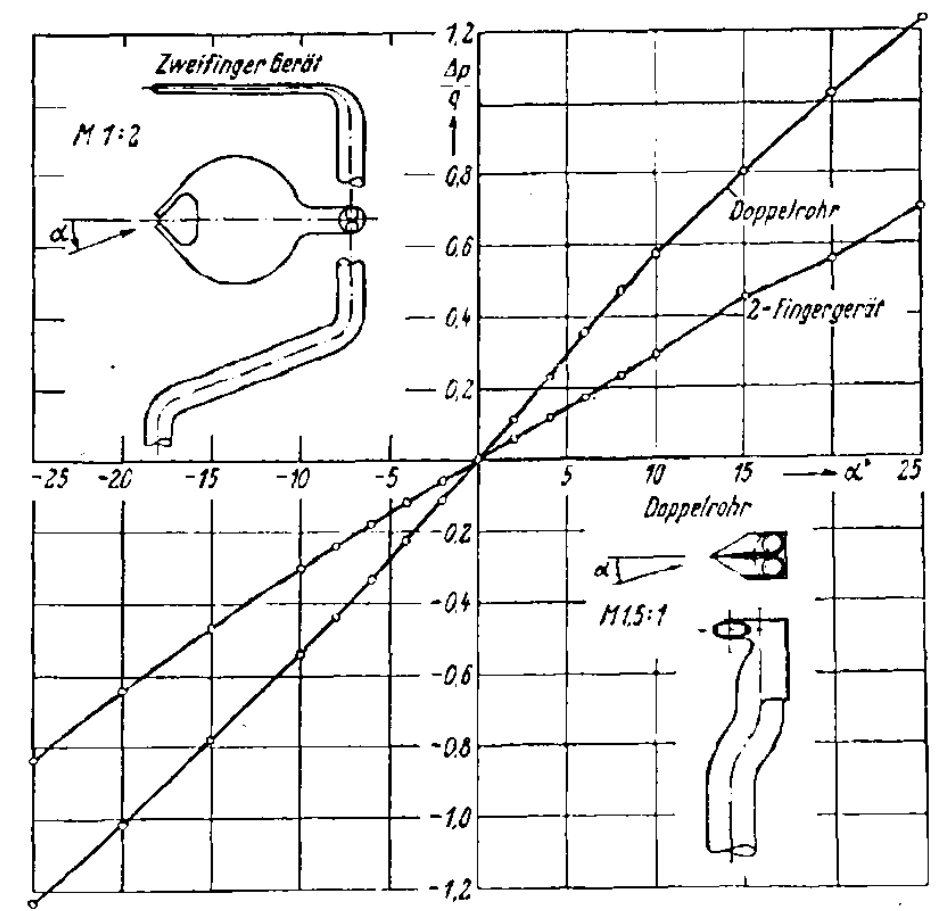

Figure 1.4 - Results from O. Conrad's original work for Conrad probe [doppelrohr] and claw-type yaw meter [Zweifinger Gerat] (courtesy of Technisches Messen, October 1950)

Conrad found that the pressure difference from tubes of this new probe varied approximately linearly with respect to their angular orientation in a flow. It was theorized that this linearity could be used measure flow angle in fluid flows of unknown direction. His experiments with the new probe showed that greater angular sensitivity could be achieved in comparison to a claw-type yaw meter, shown by the steeper slope of the doppelrohr (Conrad Probe) in comparison to the Zweifinger Gerate (claw-type yaw meter) in Figure 1.4. Later, Brebner 
[10][11] used this new type of yaw meter, which he referred to as a Conrad probe, along with a novel calibration technique to measure three-dimensional boundary layer profiles on a 59 degree sweptback wing section. His calibration used a nulling method that rotated the probe about an axis to ascertain flow angle over the test section, and then applied calibration functions to obtain velocity magnitude and static pressure on the section. His research confirmed that the Conrad probe combined with his unique calibration method could resolve yaw angles, static pressures and velocity magnitudes around the wing section. Additionally, his data indicates that the Conrad probe was able to measure the lagging flow angle variation of the span-wise velocity component within the boundary layer on swept wings. Following Brebner's work Black [12], Seddon [13], and Horlock [14] employed the Conrad probe in a similar fashion to measure the properties of two- and three-dimensional flows on a variety of wing and jet inlet configurations. Horlock utilized a nulling method to obtain flow angles only, without using Brebner's additional calibration functions. Seddon obtained a survey of the velocity distribution and flow direction in a swept leading edge intake duct using Brebner's exact calibration method. In Black's work the Conrad probe was used to explicitly obtain a varying span-wise component of velocity as a function of distance from a wing surface in the normal direction as well. Having established the Conrad probe as a viable alternative for obtaining flow angle measurements, the next step in its development was brought about by Bryer et al. [15] who tested two groups of pressure probes for use in threedimensional flow fields. 5-tube, conical, pyramid, and axial probes were tested to 
quantify measurement of flow pitch, yaw, and velocity. 2-tube Conrad, chisel, and 3-tube Conrad probes were tested in boundary layers to quantify measurement of flow yaw angle and velocity. It was concluded from the threedimensional boundary layer results that the 2-tube Conrad probe was the most useful for obtaining boundary layer measurements due to its ease of manufacture, pitch insensitivity, and low error. Results from specific probes were compared by checking their individual sensitivities to yaw angle. Selected sensitivity results are shown in Table 1.2 below.

Table 1.2 - Results from Bryer and Pankhurst for Conrad probe sensitivity versus included probe angle

\begin{tabular}{|c|c|c|c|}
\hline Probe Type & Source & $\begin{array}{c}\text { Included } \\
\text { Angle }\end{array}$ & $\begin{array}{c}\text { Angular } \\
\text { Sensitivity }\end{array}$ \\
\hline Conrad, two-hole & Bryer \& Pankhurst [16] & $\theta=60^{\circ}$ & 0.050 \\
\hline Conrad, two-hole & Bryer \& Pankhurst [16] & $\theta=70^{\circ}$ & 0.049 \\
\hline Conrad, two-hole & Bryer \& Pankhurst [16] & $\theta=120^{\circ}$ & 0.031 \\
\hline Conrad, two-hole & O. Conrad [9] & $\theta=60^{\circ}$ & 0.057 \\
\hline Conrad, two-hole & G.G. Brebner [10] & $\theta=70^{\circ}$ & 0.067 \\
\hline
\end{tabular}

From the sensitivity results in Table 1.3 and Bryer's earlier work, it is seen that a Conrad probe with ID/OD ratio of 0.6 and tip included angle of $70^{\circ}$ was found to give lower pressure transmission lag and more consistently accurate results. These results were later included with those of Pankhurst in a landmark 1971 book entitled "Pressure-probe methods for determining wind speed and flow direction" [16]. The Conrad probe continued to be of use in flow angle studies in multiple areas of fluid mechanics throughout the next decade. Winkelmann utilized a Conrad probe to measure flow angles in support of hotwire surveys of vortices created by the edges of 3-dimensional swept wings 
[17][18]. Another application was realized in supersonic flow; experiments performed by Davis [19][20][21] with supersonic flow in a square duct used a rotating Conrad probe in a nulling configuration to establish local velocity direction.

With the continuing advancement of traverse technology with higher accuracy and more degrees of freedom, the use of Conrad probes in a nulling configuration became more practical as a quick way to ascertain flow angle.

Sforza et. Al [22] utilized such a traverse system to measure surface curvature effects on boundary layer development. Frey [23] used a Conrad probe to measure flow direction in order to determine the orientation of a Pitot probe, from which velocity magnitude was measured. Towards the latter half of the 1990's and into the next decade, the techniques for using Conrad probes varied more toward the use of non-nulling calibrations that took advantage of a known flow field dynamic pressure or a center tube to measure total pressure and thus only needed to resolve flow angles. Highlights of this are experiments by Gooden et.al [24] with three dimensional flows on swept wings, and Hold et. al [25] with combined flat plate and suction flow experiments. Experiments using the threehole Conrad probe variation were conducted by Hin Ho [26] in jet intake flows and also by Bradshaw [27] while studying the effects of turbulence on pressure probes where the center tube was used to obtain total pressure and thus velocity magnitude. A summary of the calibration methods used by the various authors mentioned appears in Table 1.3 below, along with the form of their calibration functions. 
Table 1.3 - Comparison of probe calibration methods from various sources

\begin{tabular}{|c|c|c|c|c|c|}
\hline Reference & Author(s) & $\begin{array}{c}\text { Type of } \\
\text { Calibration }\end{array}$ & $\begin{array}{l}\text { Components } \\
\text { Measured }\end{array}$ & $\begin{array}{l}\text { Form of } \\
\text { Calibration } \\
\text { Functions }\end{array}$ & $\begin{array}{c}\text { Flow } \\
\text { Measured }\end{array}$ \\
\hline [9] & Conrad, $\mathrm{O}$. & Nulling & Yaw Angle & $\frac{P_{L}-P_{R}}{q}$ & $\begin{array}{c}\text { General } \\
\text { calibration }\end{array}$ \\
\hline [10], [11] & Brebner, G.G. & Nulling** & $\begin{array}{c}\text { Yaw Angle } \\
\text { Velocity } \\
\text { Magnitude }\end{array}$ & $\begin{array}{c}\frac{P_{L}-P_{R}}{q} \text { and } \\
\frac{P_{A V G}}{q}\end{array}$ & $\begin{array}{l}\text { Swept } \\
\text { wing test } \\
\text { sections }\end{array}$ \\
\hline [12], [13] & $\begin{array}{c}\text { Black, J.; } \\
\text { Seddon, J } \\
\text { and Trebble } \\
\text { W.J.G. }\end{array}$ & Nulling** & $\begin{array}{l}\text { Yaw Angle } \\
\text { Velocity } \\
\text { Magnitude }\end{array}$ & $\begin{array}{c}\frac{P_{L}-P_{R}}{q} \text { and } \\
\frac{P_{A V G}}{q}\end{array}$ & $\begin{array}{l}\text { Swept } \\
\text { wing test } \\
\text { sections; } \\
\text { Swept } \\
\text { leading } \\
\text { edge } \\
\text { intakes; }\end{array}$ \\
\hline [26] & Hin Ho, S.S. & Non-nulling & $\begin{array}{l}\text { Yaw Angle } \\
\text { Velocity } \\
\text { Magnitude* }\end{array}$ & $\begin{array}{c}\frac{P_{1}-P_{3}}{P_{n}} \text { and } \\
\frac{P_{2}-P}{P_{n}} \\
P_{n}=P_{2}-\frac{1}{2}\left(P_{1}+P_{3}\right)\end{array}$ & Jet Intakes \\
\hline [28] & Prahlad, T.S. & Non-nulling & Yaw Angle & $\frac{P_{L}-P_{R}}{q}$ & $\begin{array}{l}\text { General } \\
\text { Calibration }\end{array}$ \\
\hline [27] & $\begin{array}{c}\text { Christiansen, } \\
\text { T and } \\
\text { Bradshaw, } \mathrm{P} .\end{array}$ & Non-nulling & $\begin{array}{l}\text { Yaw Angle } \\
\text { Velocity } \\
\text { Magnitude* }^{*}\end{array}$ & $\begin{array}{c}\frac{P_{1}-P_{3}}{P_{n}} \text { and } \\
\frac{P_{2}-P}{P_{n}} \\
P_{n}=P_{2}-\frac{1}{2}\left(P_{1}+P_{3}\right)\end{array}$ & $\begin{array}{l}\text { Turbulent } \\
\text { flow }\end{array}$ \\
\hline
\end{tabular}

* - Three-hole Conrad probe used

** - Calibration functions used to find total head and static pressure

From the various uses of the Conrad probe over its history, a variety of different calibration methods were used to obtain required flow measurements. Each calibration method was tailored to the individual experiments conducted 
and also to the types of measurements required. These experiments presented a different set of known and unknown measurements than those needed in the present, in that they did not explicitly calculate the local dynamic pressure, $\frac{q}{q_{R E F}}$ (which is converted to velocity magnitude, $\frac{U}{U_{R E F}}$ ); but instead used either a separate Pitot tube or center tube. In experimental configurations where the static pressure is a known quantity and the local dynamic pressure is a parameter to be measured with a two-hole Conrad probe instead of a separate tube, a different type of non-nulling calibration is needed in order to account for this additional degree of freedom.

The objective of this thesis was to implement a two-hole Conrad probe for use with the boundary layer data system that would employ a calibration technique that would measure both flow yaw angle and velocity magnitude without the need to rotate the probe about an axis in a nulling manner. This end was to be brought about with the completion of four main objectives:

- To develop a probe design and calibration scheme for a two-hole Conrad probe that would measure both flow velocity magnitude and direction.

- To interface the two-hole Conrad probe with the hardware and software components of the BLDS unit

- To perform developmental testing and validation of the two-hole Conrad probe in a controlled wind tunnel environment.

- To utilize the Conrad probe and BLDS unit in a flight test application for demonstration purposes. 


\section{PROBE DESIGN AND CALIBRATION}

The fabrication of multi-holed pressure probes requires attention to accuracy in both the finish at the ends of the tubes and in the positioning of the tubes during assembly. Nickel or stainless steel tubes which allow close fitting, and soldered joints are accepted as standard for most pressure probes [16]. The tip of the Conrad probe was constructed with two hardened thin walled stainless steel tubes with an optimal OD/ID ratio of 0.6. As shown in Figure 2.1, these tubes were placed side-by-side and silver soldered together down their length. The smaller tubes transition via soldered joints to larger diameter tubes that run the length of the probe and end in bulged tabulations. After the length of the tubes and their joints were silver soldered, each end was ground to an oblique angle of approximately $45^{\circ}$, giving an included angle of $90^{\circ}$ for the tip. The tip angle on the Conrad probe has varying effects on the sensitivity of flow angle and dynamic pressure sensitivity. A smaller tip included angle yields a larger flow angle sensitivity at the price of a lower dynamic pressure sensitivity, while a larger tip included angle decreases the flow angle sensitivity with the benefit of increased sensitivity to dynamic pressure [15] [16]. A tip included angle of $45^{\circ}$ was chosen to ensure a greater dynamic pressure sensitivity, at the

price of lesser angular sensitivity, so that measured values of $\frac{q}{q_{R E F}}$ would be readable by the pressure sensors for the Conrad probes' future use on the device. 


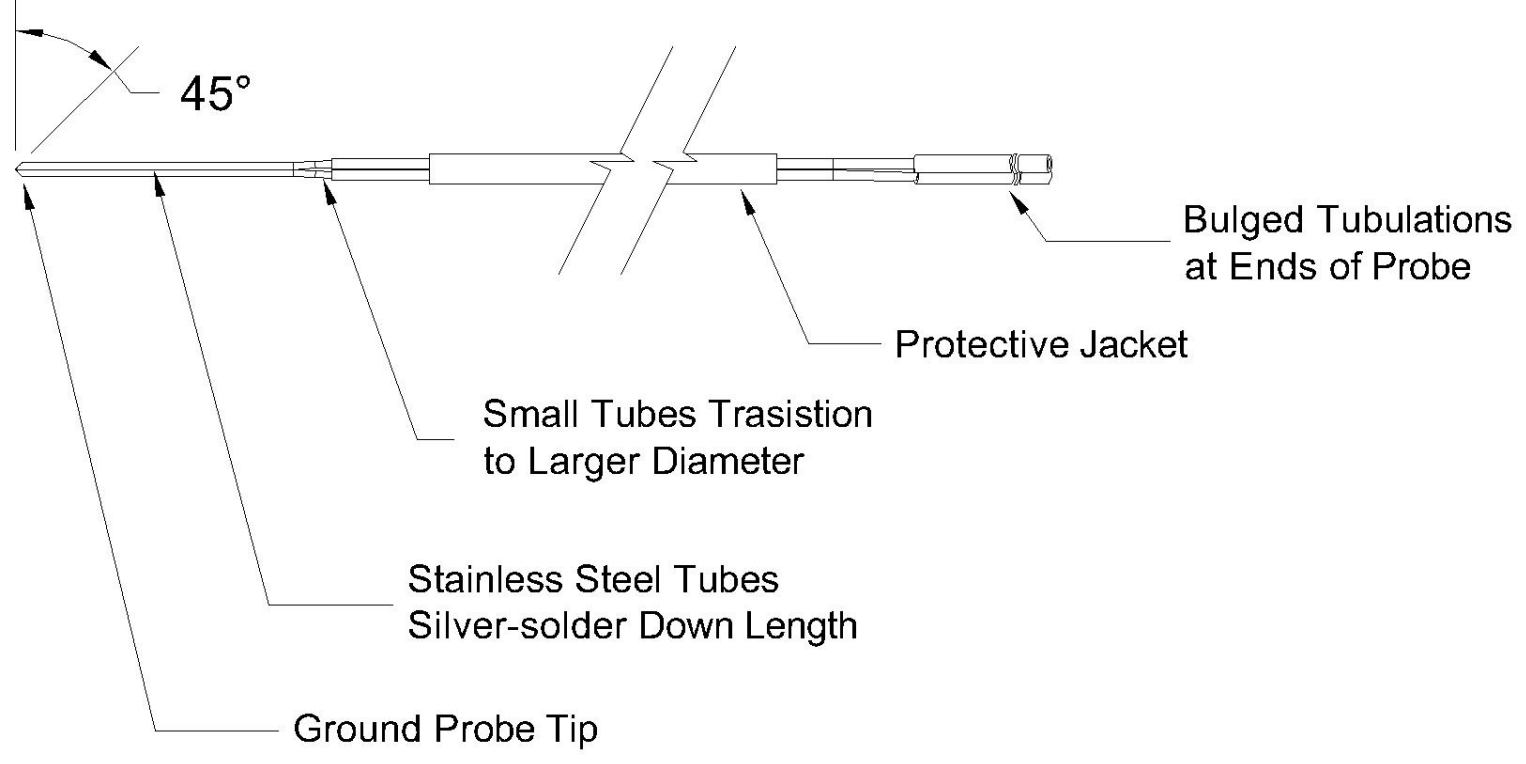

Figure 2.1 - Diagram of Conrad probe

The prototype Conrad probe was constructed using 16 gauge $(0.064 "$ outer diameter, 0.047 " inner diameter) SS304 stainless steel tubing. This probe was made for initial calibration and testing to show proof of concept. A brass jacket was attached to the outside in order to protect the thin tubing during testing. The finished probe is shown in Figures 2.2 and 2.3 below.

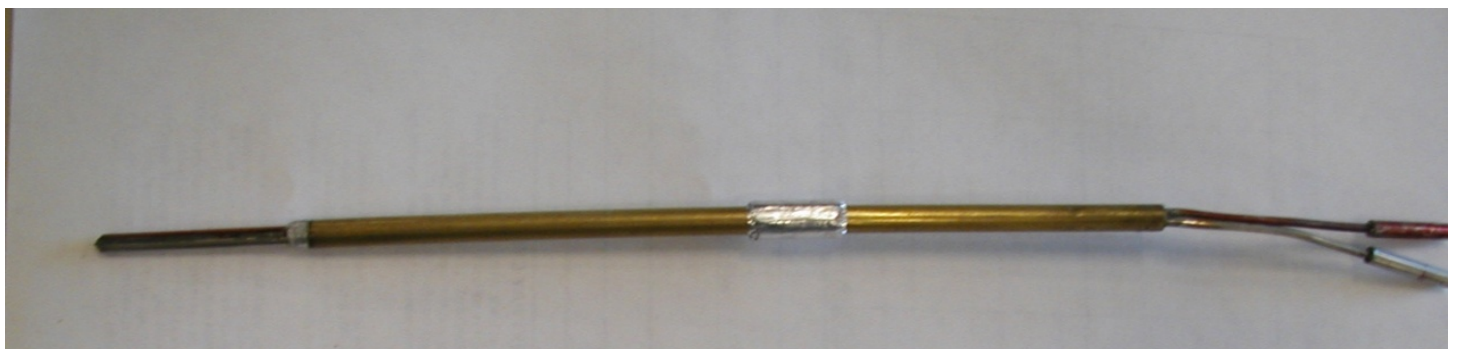

Figure 2.2 - 0.064" Prototype Conrad probe 


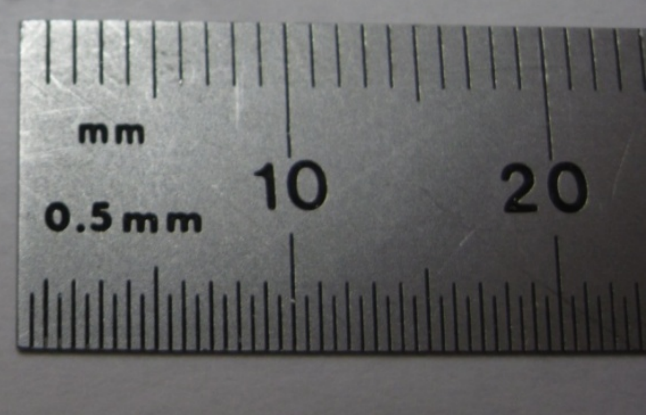

Figure 2.3 - Tip of 0.064 " diameter Conrad probe

After initial testing and calibration was performed on the first prototype, a smaller Conrad probe was constructed to eventually be used on the BLDS unit for in-flight testing. The smaller Conrad probe was constructed with 25 gauge thin wall (0.020" outer diameter, 0.012 " inner diameter) SS304 stainless steel tubes with a tip included angle of $90^{\circ}$. Figures 2.4 and 2.5 show the second iteration of the Conrad probe design.

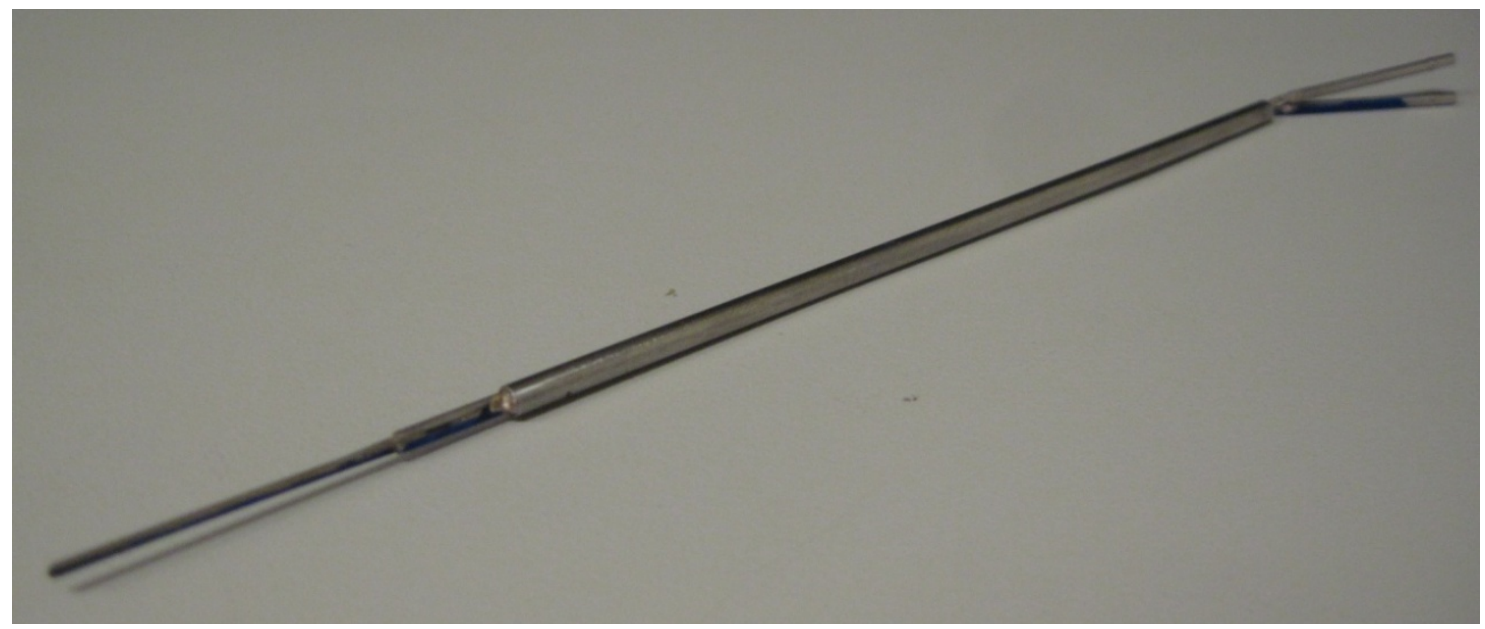

Figure 2.4-0.020" diameter Conrad probe 


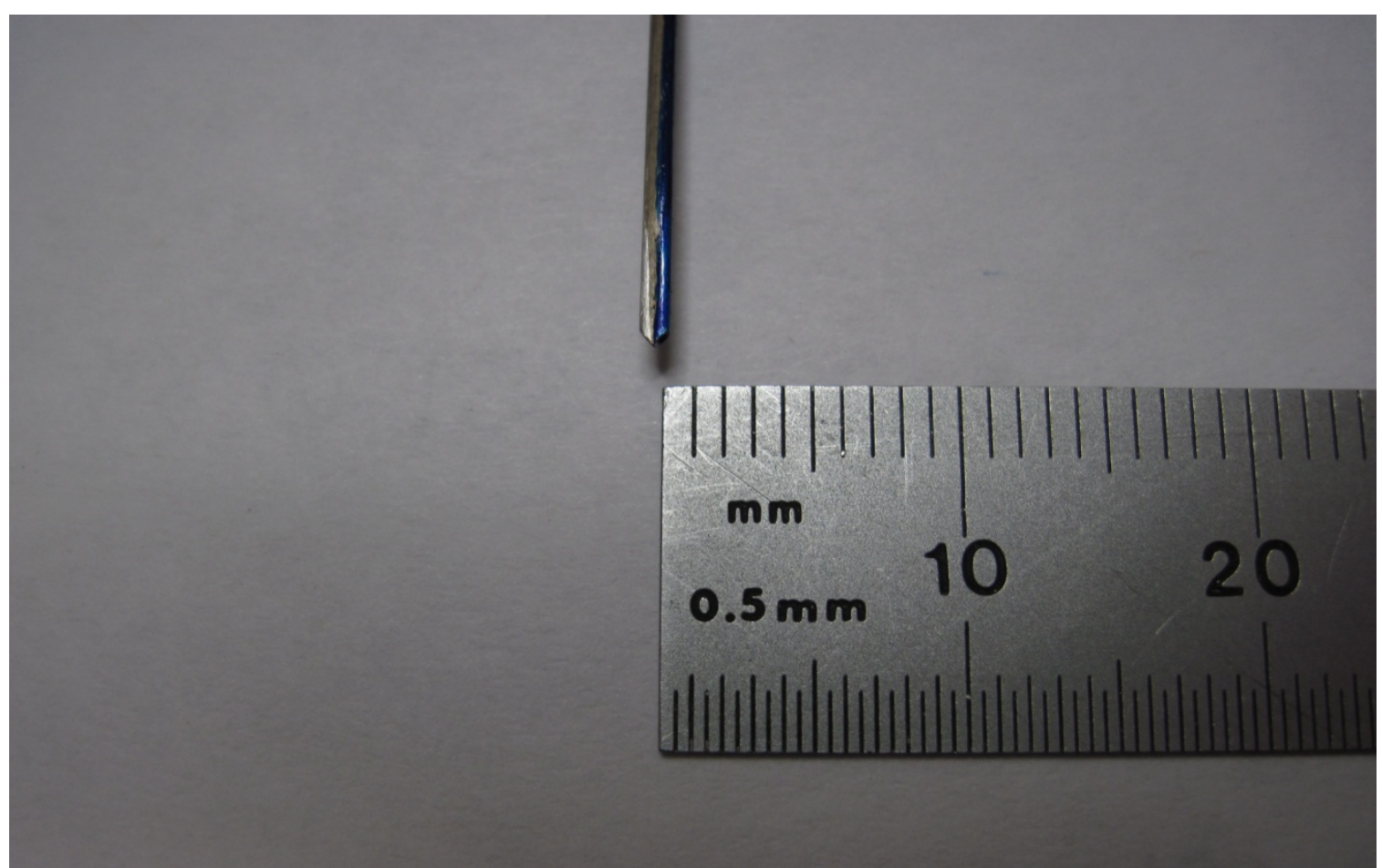

Figure 2.5 - Tip of 0.020 " diameter Conrad probe with $\mathrm{mm}$ scale

With the prototype probes constructed, a calibration scheme was needed in order to utilize the probes for measurements in a boundary layer. The approach to the calibration of any pressure probe is based on the flow parameters that are required to be resolved in order to get a full understanding of the flow field behavior. In the case of the Conrad probe, the flow field to be measured was the boundary layer of a finite length swept wing. The boundary layer over a swept wing can be highly three-dimensional and is usually broken into the components $\mathrm{U}, \mathrm{V}$, and $\mathrm{W}$ that act in the orthogonal directions $\mathrm{x}, \mathrm{y}$, and $\mathrm{z}$ respectively, with $x$ aligned nominally to the stream-wise direction on the wing. Under certain conditions boundary layer development in these three orthogonal directions is shown to occur [4]. It becomes useful in aerodynamic applications where measurements are carried out near enough to a surface, to neglect the 
component of the velocity $\mathrm{V}$ in the $\mathrm{y}$-direction (perpendicular to the surface) in order to simplify analysis and measurement of the three dimensional boundary layers. This can be accomplished if the velocity vector in the direction perpendicular to the surface is vanishingly small or of negligible magnitude when compared to the stream-wise $(U)$ and span-wise (W) velocities [4][16]. These two velocity components are related to one another in terms of the "yaw-angle" which is most often designated with the symbol $\beta$. A graphical representation of this is shown below in Figure 2.6.

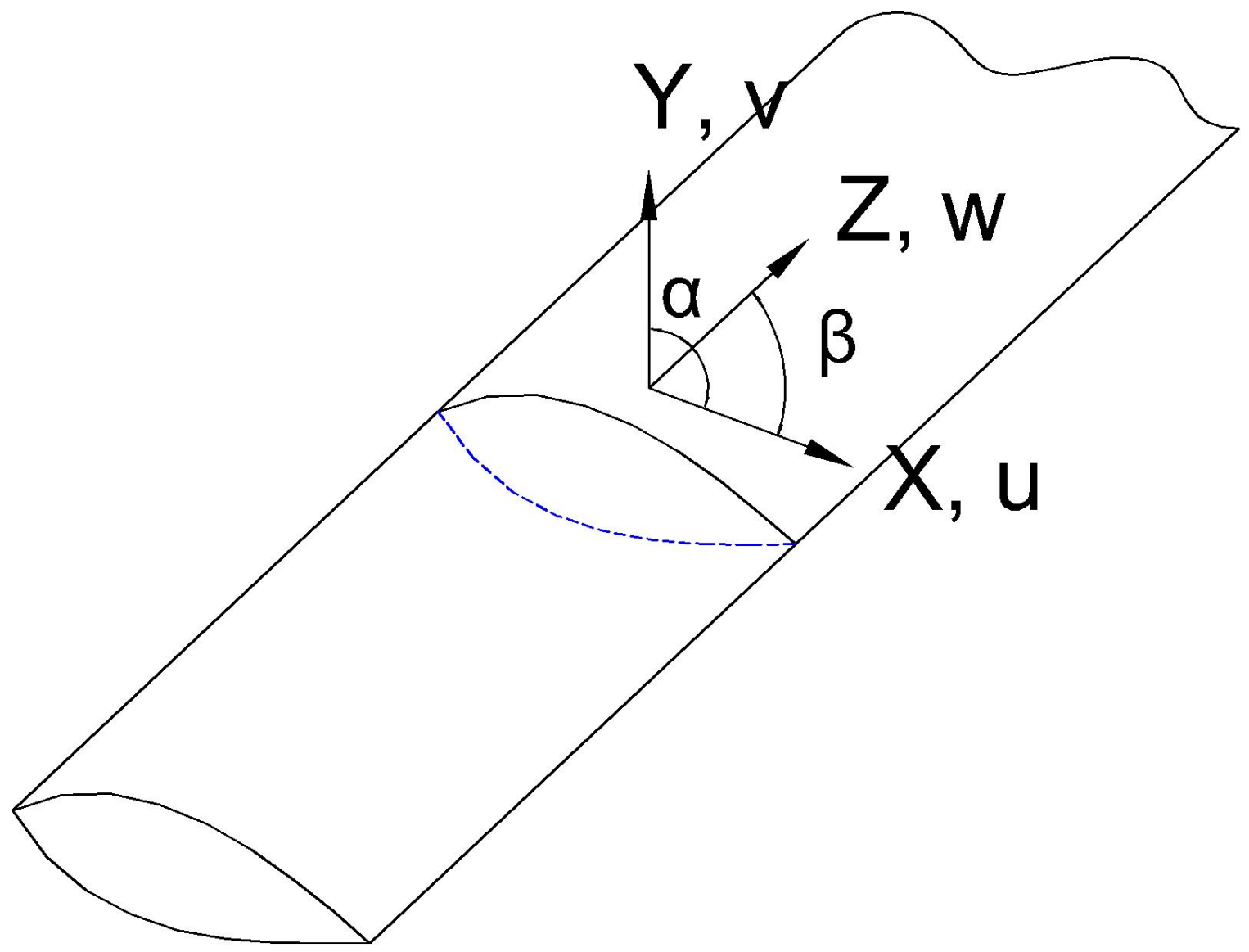

Figure 2.6 - Swept wing geometry in terms of $U$ and W 
As discussed previously in Chapter 1, Conrad's original probe [9] resolved the angle of flow by measuring the pressure difference between two chamfered tubes of the Conrad probe. Using his calibration method, the pressure difference is recorded while rotating the probe in-plane until both tubes read the same pressure, at what is called the "null point". The angle of the aligned probe is then measured with respect to some arbitrary zero point. The dynamic or total pressure is ascertained at this "null point" by use of a Pitot probe and the velocity magnitude of the flow is found accordingly [16].

An additional method utilizes a probe that had been calibrated in a flow field of known velocity and flow angle before being placed in the flow field to be measured. This calibration is done by recording pressures from both of the tubes of the probe at known flow velocities and probe angular orientations. These pressure-velocity and pressure-angle relations are then synthesized into sets of non-dimensional pressure coefficients that can be subjected to polynomial fits to make calibration functions that describe the behavior of the measured pressures over the known flow angles and velocity magnitudes. After calibration in this manner, the probe is placed in an unknown flow field of interest and the pressures in the tubes are recorded. The calibration functions are then inverted to obtain the flow angle and velocity magnitude. This method is known as the "non-nulling" calibration method.

Each method has advantages depending on the conditions, location, available hardware, time allotted for measurement, and the measurements needed. In terms of a laboratory setup with long data acquisition time and a 
positioning system capable of translation and rotation, the nulling method is a superior way to obtain flow angles due to the fact that a probe can immediately be used and calibrated in the measured flow field. However, this method presents unique challenges for field implementation, as it requires significant time along with some trial and error to find a null point. Another challenge is found with fixing the tip of a probe in a location while still being able to rotate in-plane to find the null point, which requires a traverse system with the ability to move in very fine increments of angle and to measure the flow angle accurately with respect to a given reference point.

The non-nulling method is somewhat more demanding due to the more extensive calibration needed and the additional uncertainty that this calibration can introduce to a measurement scheme. The probe must be carefully placed in some sort of known flow field as described previously and must also be tested at various velocities to ensure proper results are obtained when the probe is in the field. The extensive calibration does make this method much more versatile in the field; the probe in question can be put into the unknown flow field and made to resolve flow angle and magnitude with respect to any arbitrary orientation. Also, due to the fact that the probe has no need to establish a null point, it can remain fixed in an orientation and still deliver flow data making it useful for locations that would be difficult to reach using a probe capable of nulling. Based on these details, the non-nulling method was chosen for use with the Conrad probe on the BLDS device. 
Calibration of the Conrad probe was carried out with the use of two calibration functions and two non-dimensional pressure coefficients. The pressure coefficients are defined in terms of the following pressures:

$$
\begin{aligned}
& P_{L}=P_{\text {Left tube }}-P_{\text {static }} \\
& P_{R}=P_{\text {Right tube }}-P_{\text {static }} \\
& P_{L-R}=P_{\text {left tube }}-P_{\text {right tube }} \\
& P_{A V G}=\frac{1}{2}\left(P_{L}+P_{R}\right)=P_{L}-\frac{1}{2}\left(P_{L-R}\right) \\
& q=P_{\text {dynamic }}=P_{\text {total }}-P_{\text {static }} \\
& \mathrm{P}_{\mathrm{R}}
\end{aligned}
$$
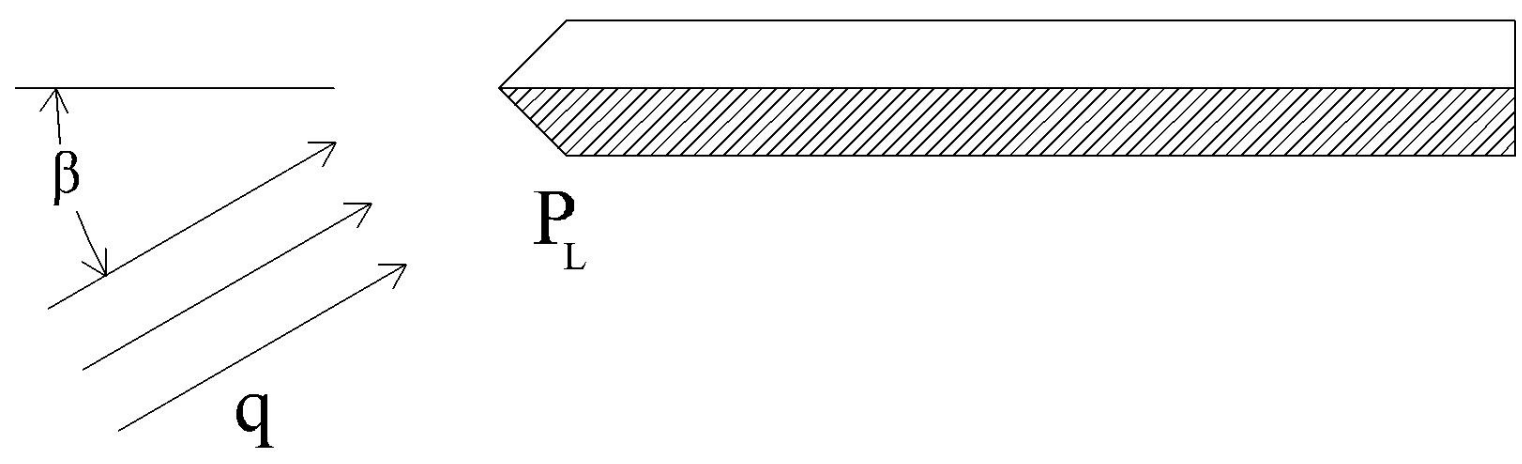

$\mathrm{P}_{\mathrm{L}}$

Figure 2.7 - Diagram of Conrad probe and measured quantities

Using the two measured pressure differences and the dynamic pressure, two non-dimensional pressure coefficients were defined: 


$$
\begin{gathered}
C_{P A V G}=\frac{P_{L}-\frac{1}{2}\left(P_{L-R}\right)}{q}=\frac{P_{A V G}}{q} \\
C_{P \Delta}=\frac{P_{L-R}}{P_{A V G}}
\end{gathered}
$$

These two dimensionless pressure coefficients are assumed to be functions of yaw angle only, allowing the following calibration functions to be defined:

$$
\begin{gathered}
C_{P A V G}=\frac{P_{L}-\frac{1}{2}\left(P_{L-R}\right)}{q}=f(\beta) \\
C_{P \Delta}=\frac{P_{L-R}}{P_{A V G}}=g(\beta)
\end{gathered}
$$

Values of the pressure coefficients for a yaw range $-30^{\circ} \leq \beta \leq 30^{\circ}$ were then obtained with the use of a calibration jet (TSI model 1128). With a $20 \mathrm{~mm}$ diameter jet nozzle, the calibration jet had a dynamic pressure range of 2 psf to 30 psf. To record the pressures, a Setra Model 239 pressure transducer with a range of 0 to $15 \mathrm{inWC}$ was used, along with a Fluke $8050 \mathrm{a}$ digital multimeter.

Figure 2.8 - Conrad probe positioned for testing above $20 \mathrm{~mm}$ jet 
An angular positioning fixture capable of moving the probe to $\pm 30^{\circ}$ in $6^{\circ}$ increments was used to orient the probe with respect to the jet. A photograph of the calibration setup is shown in Figure 2.9:

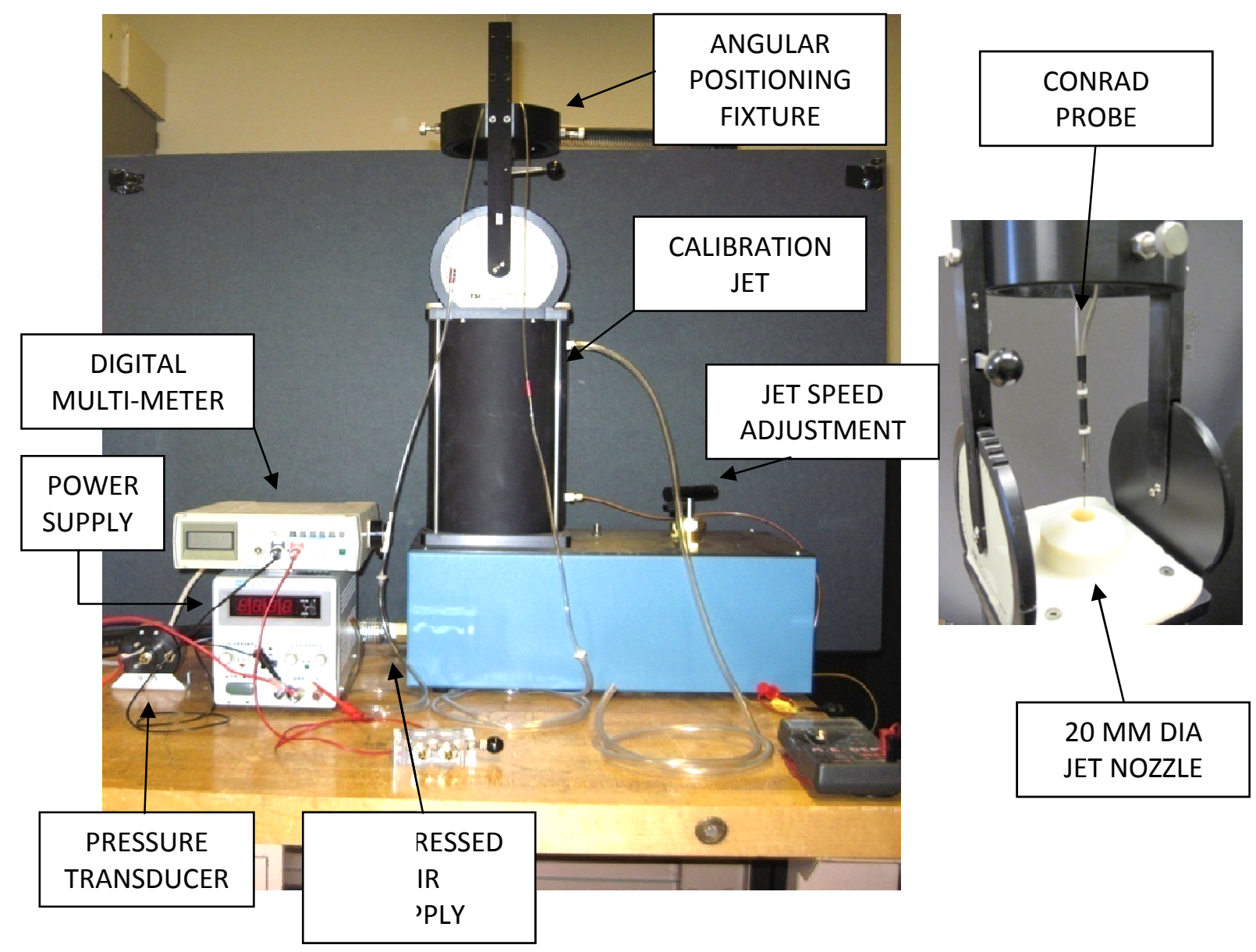

Figure 2.9 - (left) Calibration jet setup with 0.020" diameter Conrad probe (right) Calibration jet with probe on angular adjustment fixture 


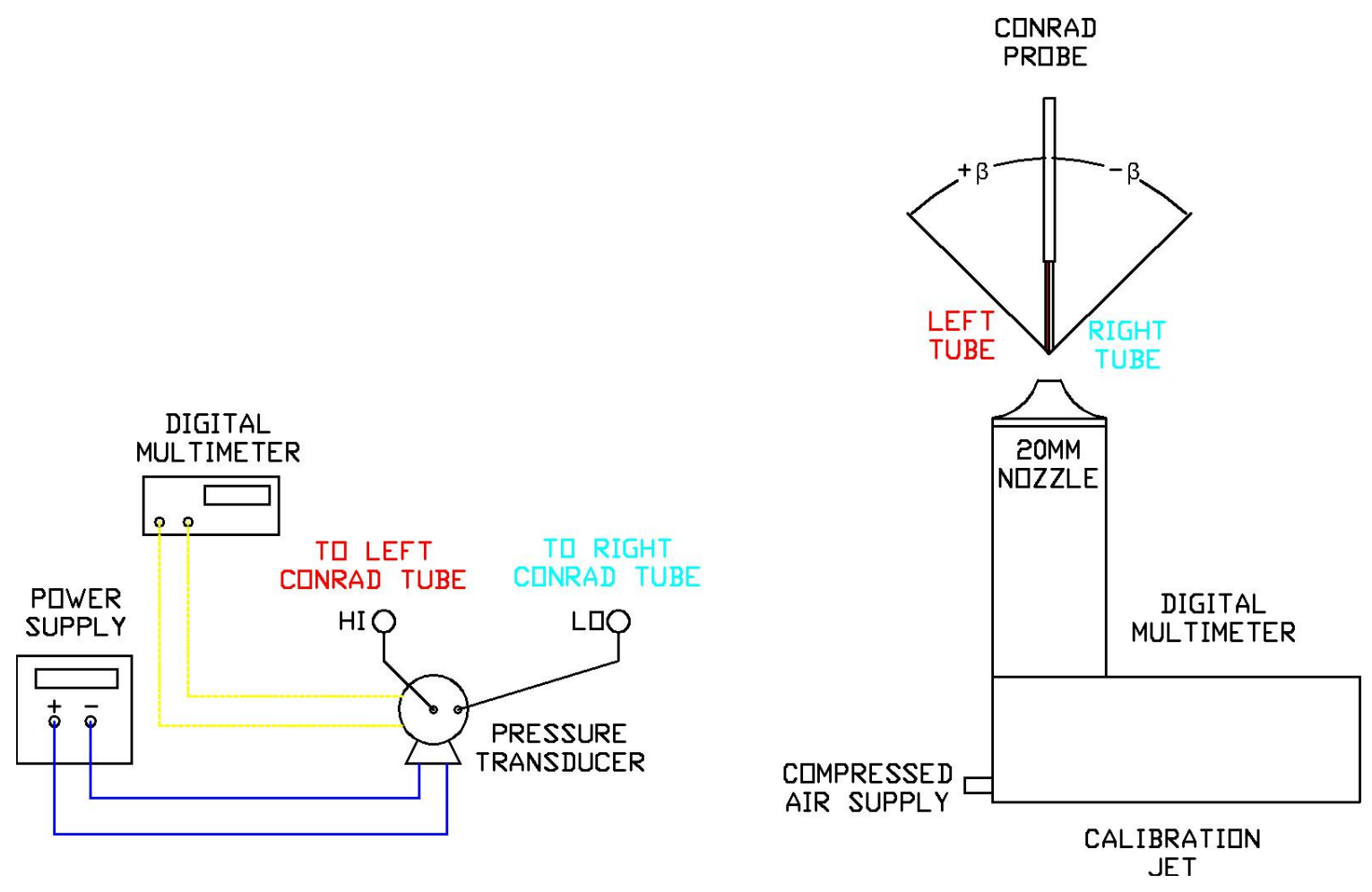

Figure 2.10 - Diagram of calibration setup

Pressure data was taken at jet velocities of 90,127 , and $155 \mathrm{ft} / \mathrm{s}(10,20$, and $30 \mathrm{psf}$ ) and was subsequently used to find values for the non-dimensional pressure coefficients. These pressure coefficient vs. yaw angle data sets were subjected to polynomial fits using the least squares regression technique to yield the calibration functions. The values of $\mathrm{C}_{P}$ AVG obtained at varying yaw angles yielded the following results shown in Figure 2.11: 


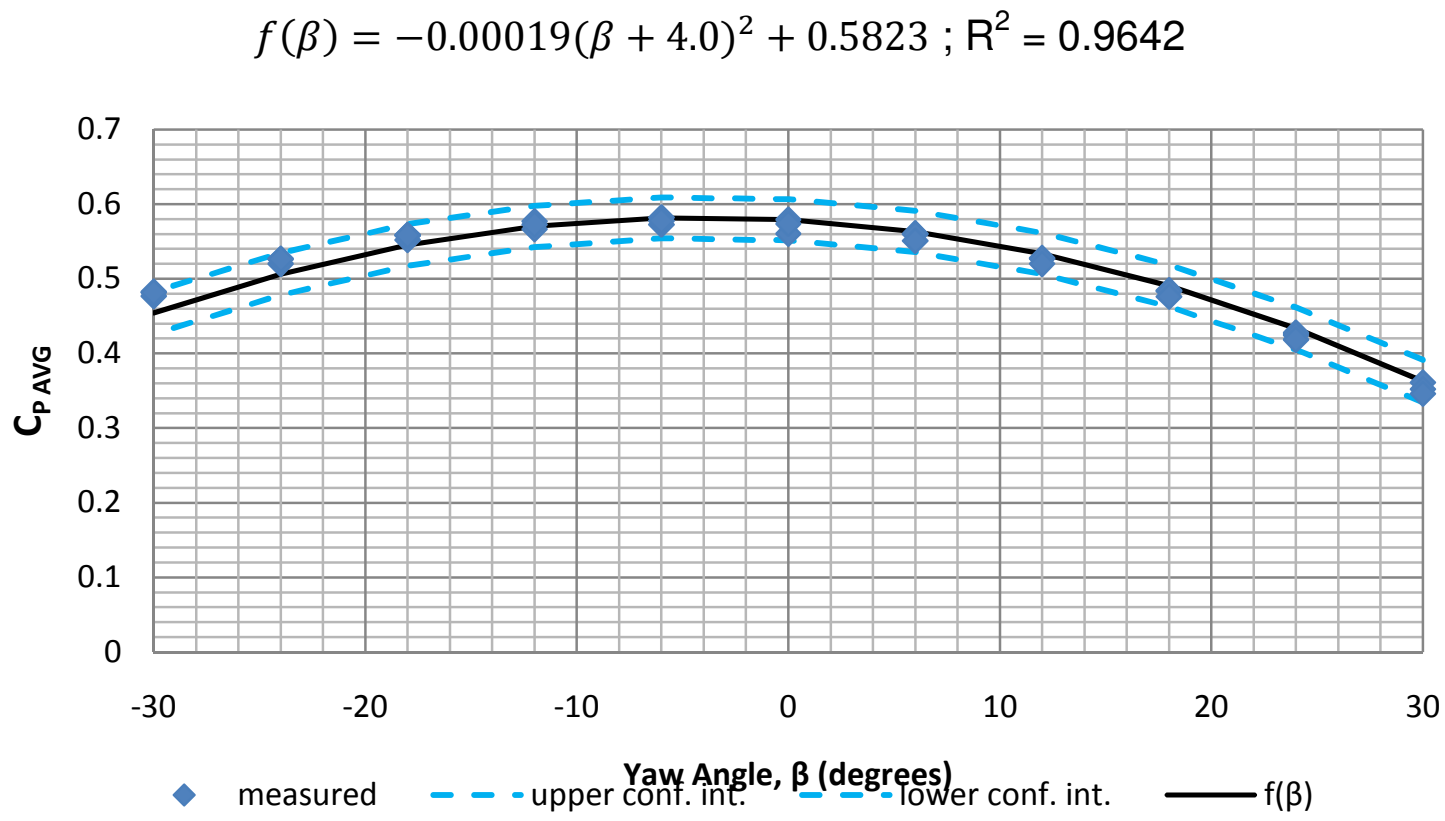

Figure $2.11-C_{P}$ AVG vs. $\beta$ for 0.020 " diameter Conrad probe

Using the non-dimensionalized average pressure for the first fit function left the calibration easy to interpret in realistic terms. The second order fit to the data was a negative symmetric even function with a maximum value for $C_{P A V G}$ of 0.58. This average pressure meant that the pressure read by the probe was $58 \%$ of maximum when the Conrad probe was placed with its axis perpendicular to the flow; in comparison a Pitot probe would have a $C_{P \text { AVG }}$ value of 1 due to its measuring $100 \%$ of the oncoming flow. It follows that the values for $\mathrm{C}_{\mathrm{P} A V G}$ is expected to be symmetric about zero degrees due to the probe being symmetric from a manufacturing standpoint. The values tapered off to smaller amounts as the probe yawed in either direction and the windward tube became exposed to more of the flow. The added angle offset to the $\beta$ term was used for two reasons; first, to compensate for manufacturing inconsistencies in the angular positioning fixture, namely the zero position on the fixture was not aligned directly to the jet 
axis. Secondly, the Conrad probe head was not perfectly symmetric causing an angle discrepancy when the fixture was in the zero position that resulted in a measured angle of four degrees at the nominal zero point. Both of these factors yielded an offset of four degrees, which was computed for one of the fits and forced upon both the $\mathrm{C}_{P A V G}$ and $\mathrm{C}_{P \Delta}$ fits.

The values of $\mathrm{C}_{P \Delta}$ with respect to yaw angle yielded a positive odd third order fit, shown by the equation and plot in Figure 2.12. The fit function again behaves according to the geometry of the tubes; the average pressure on the denominator of the $C_{P} \Delta$ term was a weak function of $\beta$, leaving the $P_{L-R}$ term to drive the function. According to the sign convention established, the measured $P_{L-R}$ increases with positive yaw angle, driving the $C_{P \Delta}$ function higher. The second constant in the $g(\beta)$ equation represents the sensitivity of the probe to yaw angle change with respect to the average pressure.

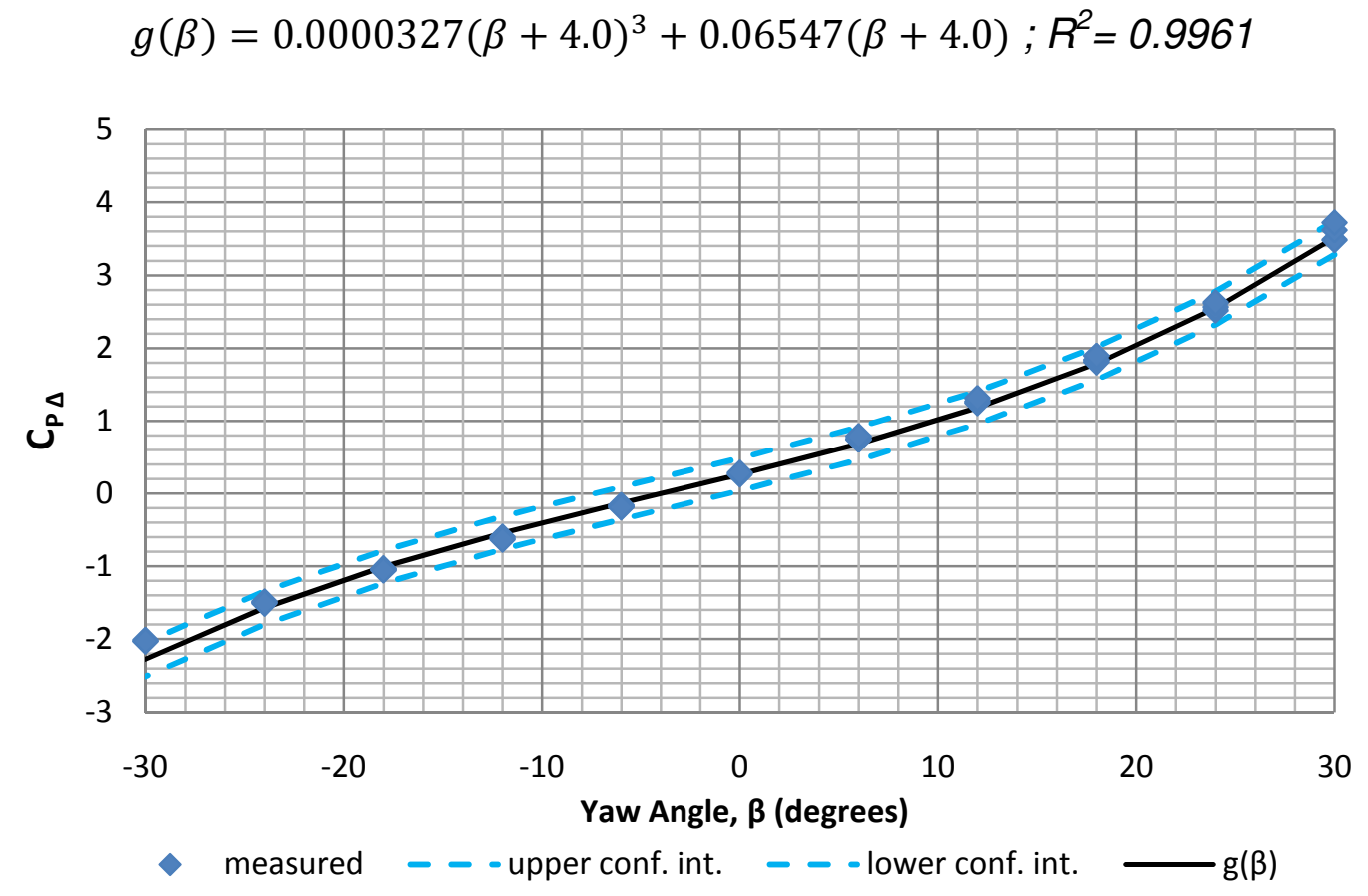

Figure 2.12 $-C_{P \Delta}$ vs. $\beta$ for $\mathbf{0 . 0 2 0 " ~ d i a m e t e r ~ C o n r a d ~ P r o b e ~}$ 
With the probe calibrated, the sensitivity of the probe in both yaw and pitch had to be checked to ensure that the probe was taking measurements in the proper range for its future boundary layer measurement application. The yaw sensitivity of a yaw-meter is defined by Bryer and Pankhurst to be [16]:

$$
\text { Yaw Sensitivity }=\frac{P_{L-R}}{q \beta}=\frac{C_{P Y A W}}{\beta}
$$

This value corresponds directly to the slope of a $C_{P \text { YAW }}$ vs. $\beta$ plot and since the yaw response of the Conrad probe is roughly linear within a \pm 30 degree range that meant the slope was easy to obtain. The yaw sensitivity measurements could be taken from the existing calibration data through manipulation of the two non-dimensional coefficients $\mathrm{C}_{\mathrm{P} \Delta}$ and $\mathrm{C}_{\mathrm{P} A \mathrm{AV}}$ :

$$
C_{P A V G} * C_{P \Delta}=\frac{P_{A V G}}{q} * \frac{P_{L-R}}{P_{A V G}}=\frac{P_{L-R}}{q}=C_{P Y A W}
$$

With the $\mathrm{C}_{\text {P YAW }}$ coefficient computed the plot shown in Figure 2.13 was obtained.

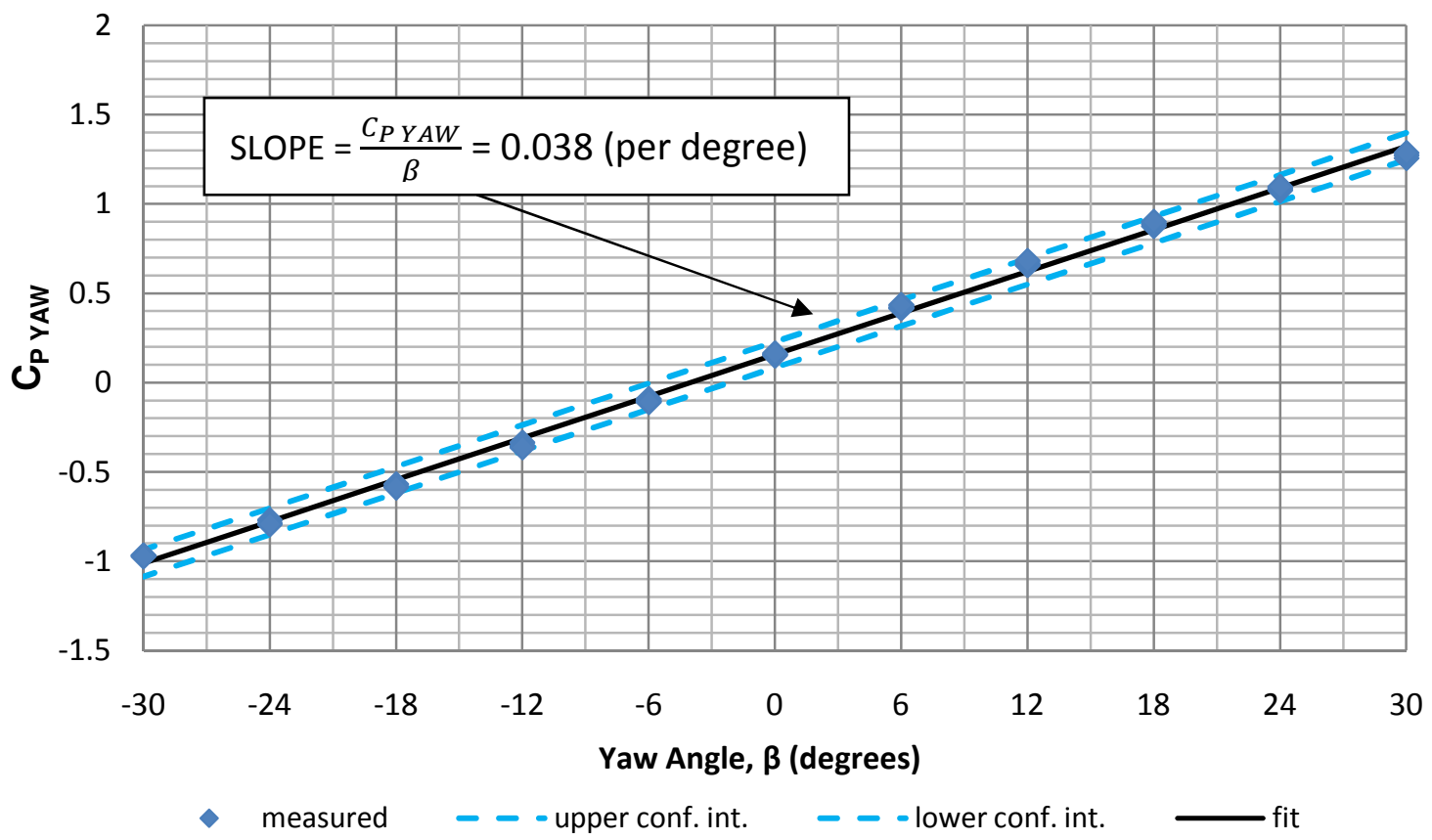

Figure $2.13-C_{\text {P YAW }}$ vs. $\boldsymbol{\beta}$ for $\mathbf{0 . 0 2 0 "}$ " diameter Conrad Probe 
The value obtained for yaw sensitivity in this way was found to be 0.038 per degree for the $90^{\circ}$ tip included angle. This value does not directly compare to published results due to a different tip angle, however it is within the range of 0.031 per degree for a $120^{\circ}$ included angle to 0.049 per degree for a $70^{\circ}$ included angle listed by Bryer and Pankhurst [16] in Table 1.2, meaning that the Conrad probe demonstrated adequate sensitivity to angular change.

For a more direct comparison of the performance of the Conrad probe and validity of the calibration, the fit functions obtained and yaw sensitivity can be compared to results obtained by Brebner [10].

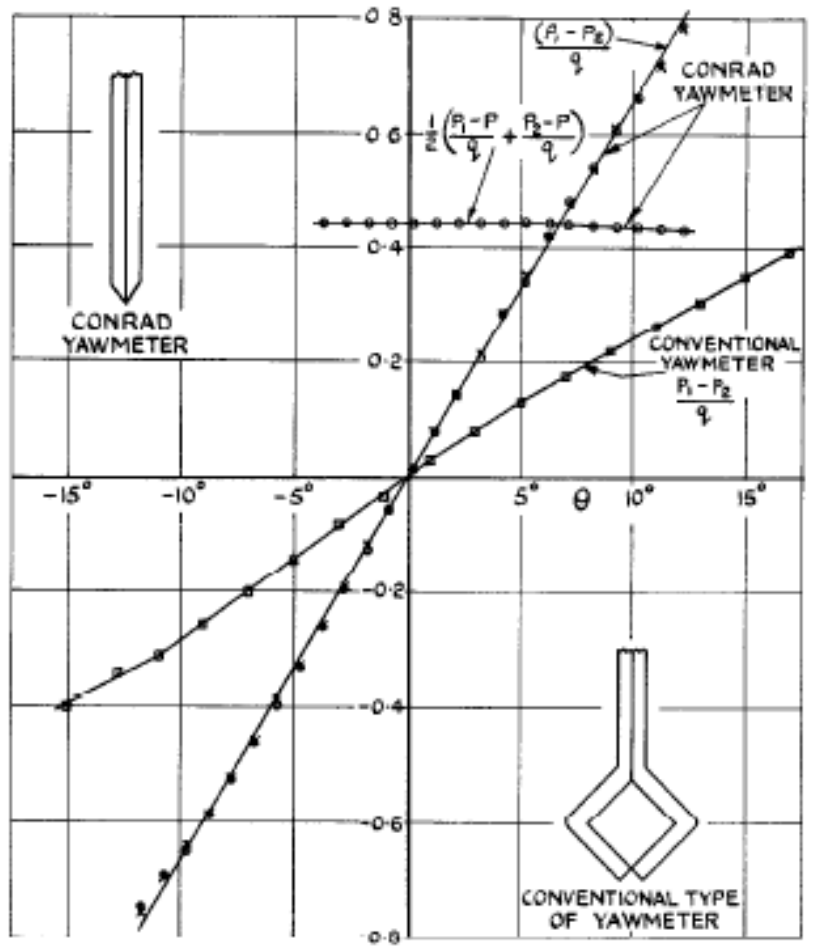

Figure 2.14 - Calibration results from G.G. Brebners' work with a Conrad probe [8]

Brebner utilized similar functions to the $C_{P}$ AVG and $C_{P \text { YAW }}$ defined in this thesis, shown above in Figure 2.14. In Brebner's case the Conrad probe used 
had a diameter of $0.04 "$, a tip included angle of $70^{\circ}$, and was calibrated over a range of $\pm 10^{\circ}$. Comparing Figures 2.11 and 2.14, the maximum value of $C_{P A V G}$ is found to be much lower at around 0.45 with Brebner's probe in comparison to the value of 0.58 for the smaller diameter $90^{\circ}$ tip probe shown in Figure 2.11. An additional difference is noted in the yaw sensitivity; the slope of Brebner's $C_{P \text { YAW }}$ curve was approximately 0.067 per degree while the slope of the curve for the 0.02 " Conrad probe was found to be 0.038 per degree. These differences highlight the effect of the probe tip included angle upon the sensitivity and range of the Conrad probe, with the smaller probe exhibiting the expected behavior of a decreased angular sensitivity but a higher dynamic pressure sensitivity.

With the calibration validated by previous results, it was necessary to explore the effect of the pitch sensitivity of the probe readings due to the fact that pitching of pressure probes is required for installation upon the BLDS device in order to position probe tips near the surface. Pitch sensitivity is defined in a similar fashion to yaw sensitivity [16]:

$$
\text { Pitch Sensitivity }=\frac{P_{L}-\frac{1}{2}\left(P_{L-R}\right)}{q_{R E F} \alpha}=\frac{C_{P A V G}}{\alpha}
$$

To measure the pitch sensitivity, the probe was placed in the calibration jet in a configuration that rotated it such that it could be pitched at various angles in a similar manner to the probe calibration. Figure 2.15 shows values for the $C_{P A V G}$ taken at different pitch angles. 


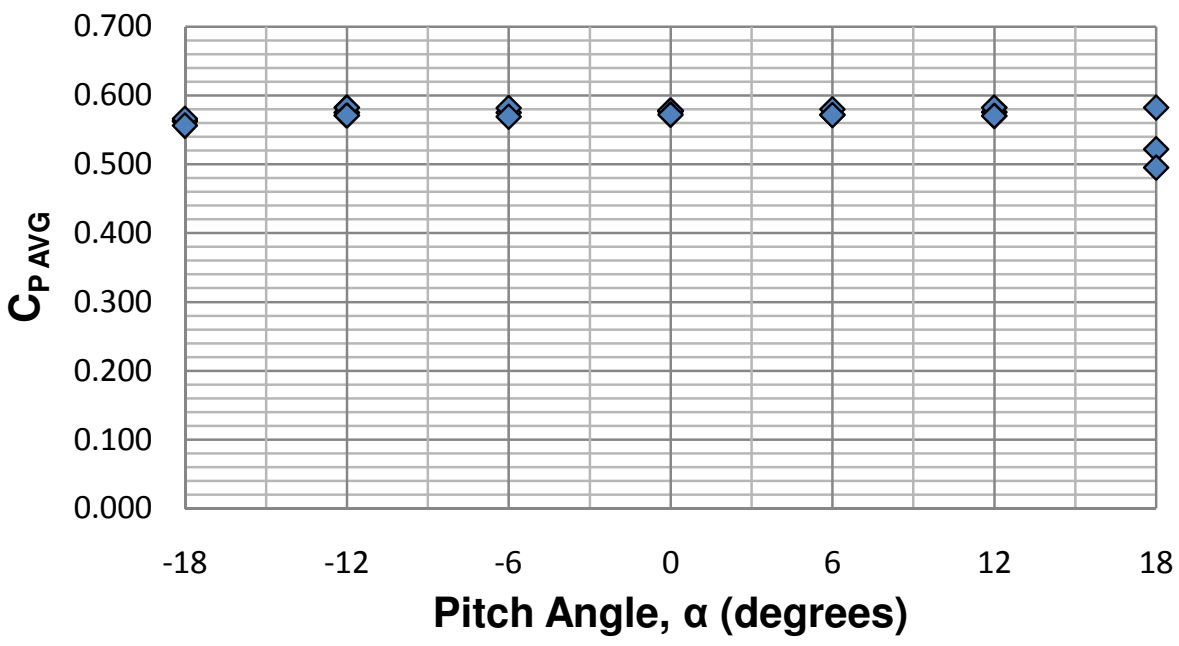

Figure 2.15 $-C_{P}$ AVG $v$ s. $\alpha$ for three calibration jet velocities

Figure 2.15 shows values of $C_{P A V G}$ at pitch angles of $\pm 18^{\circ}$ for the same three jet velocities used for initial calibration. For the full range of pitch, the value of $C_{P A V G}$ showed a deviation of greater than \pm 0.05 , or $10 \%$ of the average value. In the smaller range of $\pm 12^{\circ}$ the value of $C_{P A V G}$ shows a range of $0.58 \pm 0.003$; meaning that the readings taken by the 0.020 " diameter Conrad probe change by less than $1 \%$ within a range of $\pm 12^{\circ}$. From this the $\pm 12^{\circ}$ was deemed an acceptable range for pitch sensitivity. This range compares closely with the results of G.G. Brebner, which showed pitch insensitivity to at least $\pm 7^{\circ}$ (Brebner's results only show pitch testing to $7^{\circ}$ ) [10].

The initial calibration of Conrad probe and the confirmation of the yaw and pitch sensitivities meant that one more facet of calibration had to be addressed; uncertainty. As seen above, the flow yaw angle and the non-dimensional dynamic pressure are both dependent on three separate pressure measurements. As with any measurement scheme, these three pressures provide a source of uncertainty to any calculations involving their use. As such, it 
became necessary to quantify the propagated uncertainty involved with computing $\beta$ and $\frac{q}{q_{R E F}}$ from measurements of $P_{L}, P_{L-R}$, and $q_{R E F}$. The main method to quantify the propagated uncertainty involves partial differentiation of the equation for the computed quantity in terms of each measured quantity followed by a root sum square combination of the contribution from each measured quantity [30]. This method proved inconvenient due to the inversion of the calibration with which the computed quantities $\beta$ and $\frac{q}{q_{R E F}}$ are found from the measured ones; $P_{L}, P_{L-R}$, and $q_{R E F}$. A more convenient approximate method of error estimation, called sequential perturbation [29][30], was instead used; whereby the propagation of uncertainty in a set of measurements is estimated using a finite difference approximation of the partial derivatives of the computed quantities with respect to measured quantities. A description of the methodology and rationale behind the quantification of uncertainty for the Conrad probe is listed in Appendix D.

In addition to uncertainty for the two computed quantities, uncertainty values were also computed for velocity magnitude, $\frac{u}{u_{R E F}}$ due to the majority of boundary layer data being represented in terms of velocities. The uncertainty of the computed quantities was found to be dependent on the conditions with which the measurements were taken. In a wind tunnel test environment where the freestream dynamic pressure was roughly $15 \%$ of the range of the pressure sensors, the uncertainties for $\beta, \frac{q}{q_{R E F}}$, and $\frac{u}{u_{R E F}}$ were found to be $\pm 1.2^{\circ}, \pm 0.04$, and \pm 0.02 respectively. In the case of flight tests, with a free-stream dynamic pressure that 
was three to five times larger than that of the wind tunnel, more of the useable range of the pressure sensors was taken utilized, yielding lower uncertainties for $\beta, \frac{q}{q_{R E F}}$ and $\frac{u}{u_{R E F}}$ of $\pm 0.5^{\circ}, \pm 0.01$ and \pm 0.01 respectively. Application of the wind tunnel level uncertainties was applied to a profile of wind tunnel data in Figures 2.16 and 2.17 to show the overall effect of these uncertainties.
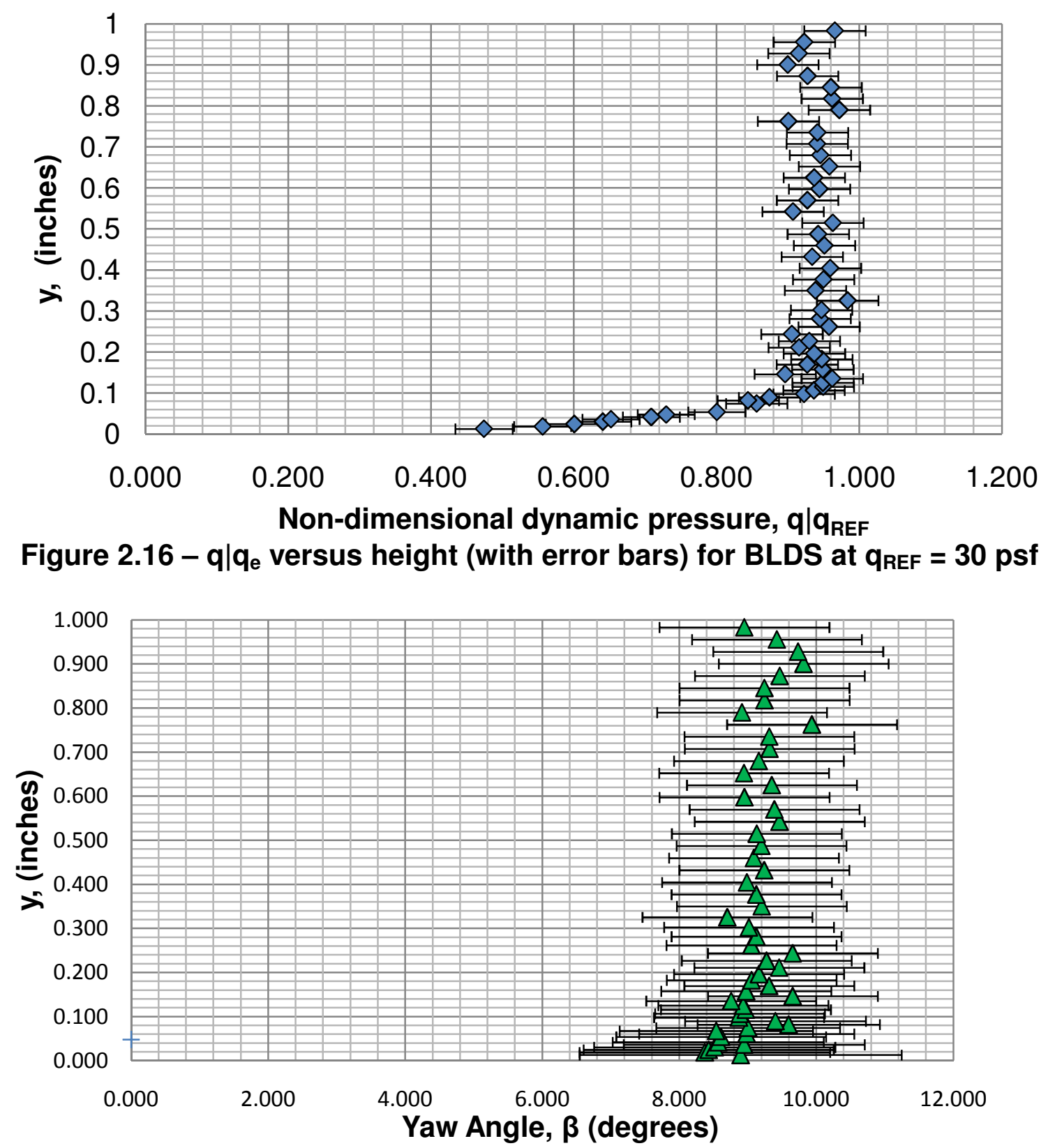

Figure $2.17-\beta$ versus height (with error bars) for BLDS at $q_{\mathrm{REF}}=30 \mathrm{psf}$ 
The uncertainty for the velocity profile shows it to be relatively uniform throughout the boundary layer. The yaw angle uncertainty was found to be uniform in the free-stream with some increased uncertainty from measurements within a few diameters of the surface. This increased uncertainty is due to the lower usage of the pressure sensor range brought about by the lower velocity both near the surface and in the wind tunnel.

After designing, building, calibrating, and validating the Conrad probe, it was ready to be incorporated into the hardware and software systems of the BLDS and tested. 


\section{DATA ACQUISITION AND ANALYSIS}

The BLDS is a mechatronic device consisting of a microcontroller subsystem controlled by proprietary software, circuit board with embedded sensors and control circuitry, a vertical traversing stage, a free-stream total pressure probe, surface static probe [31], and a battery. In order to incorporate the Conrad probe onto the boundary layer data system, changes were needed in both the hardware and software components of the device. A complete listing of the hardware capabilities of the BLDS device is below in Table 3.1.

Table 3.1 - Hardware specifications for BLDS device

\begin{tabular}{|l|l|l|}
\hline \multicolumn{1}{|c|}{ Component } & \multicolumn{1}{|c|}{ Description } & \multicolumn{1}{c|}{ Specifications } \\
\hline $\begin{array}{l}\text { Microcontroller } \\
\text { System }\end{array}$ & $\begin{array}{l}\text { TFX-11v2 Commercial } \\
\text { Dual Microcontroller }\end{array}$ & $\begin{array}{l}128 \mathrm{~KB} \text { volatile RAM } \\
2 \mathrm{MB} \text { non-volatile serial FLASH } \\
\text { memory }\end{array}$ \\
\hline $\begin{array}{l}\text { Main Circuit } \\
\text { Board }\end{array}$ & $\begin{array}{l}\text { Custom double-sided } \\
\text { printed circuit board }\end{array}$ & $\begin{array}{l}\text { One absolute and two single- } \\
\text { sided differential pressure } \\
\text { sensors; temperature sensor; } \\
\text { two motor control circuits; }\end{array}$ \\
\hline Stage & $\begin{array}{l}\text { Commercial stage } \\
\text { assembly }\end{array}$ & $\begin{array}{l}\text { Integrated DC motor, encoder, } \\
\text { and limit switches }\end{array}$ \\
\hline $\begin{array}{l}\text { Surface Static } \\
\text { Probe [31] }\end{array}$ & $\begin{array}{l}\text { Probe for measuring } \\
\text { static pressure }\end{array}$ & $\begin{array}{l}\text { Thin walled stainless tubing } \\
\text { with silver-soldered cross bar } \\
\text { and two horizontally opposed } \\
\text { holes }\end{array}$ \\
\hline Battery & Commercial Battery Pack & $\begin{array}{l}\text { LiSO } 2 \text { primary cells of spiral } \\
\text { electrode design, 1/2 AA x 3, } \\
400 \text { mAhr or full AA x 4, } \\
1000 \text { mAhr @ 8VDC }\end{array}$ \\
\hline
\end{tabular}


The BLDS hardware setup consists of a master circuit board with embedded power connection to an AA cell battery, three pressure sensors, motor controls and limit switches. Probes are moved vertically from a surface with the use of a vertical stage driven by a DC stepper motor. Probes are mounted onto the stage and plumbed with $3 / 32$ " silicone tubing to the pressure sensors [1][2]. To take readings with the Conrad probe, the three pressure sensors available on the BLDS main circuit board were used along with an additional differential pressure sensor which was connected via an auxiliary port. The three sensors on the main circuit board include two single sided differential pressure sensors with a calibrated range of 0 to 1.5 psid and one absolute sensor with a calibrated range of 0 to $15 \mathrm{psid}$, all from the 58xx line manufactured by Silicon Microstructures. The added two-sided differential pressure sensor was also from the $58 x x$ line and had a calibrated range of $\pm 1.5 \mathrm{psid}$. The new sensor was needed in order to measure the pressure difference between the left and right tubes of the Conrad probe; depending on the direction of flow, the pressure $P_{L-R}$ can be either a positive or negative value. The pressure plumbing for these sensors on the BLDS unit is shown in the diagram below. 


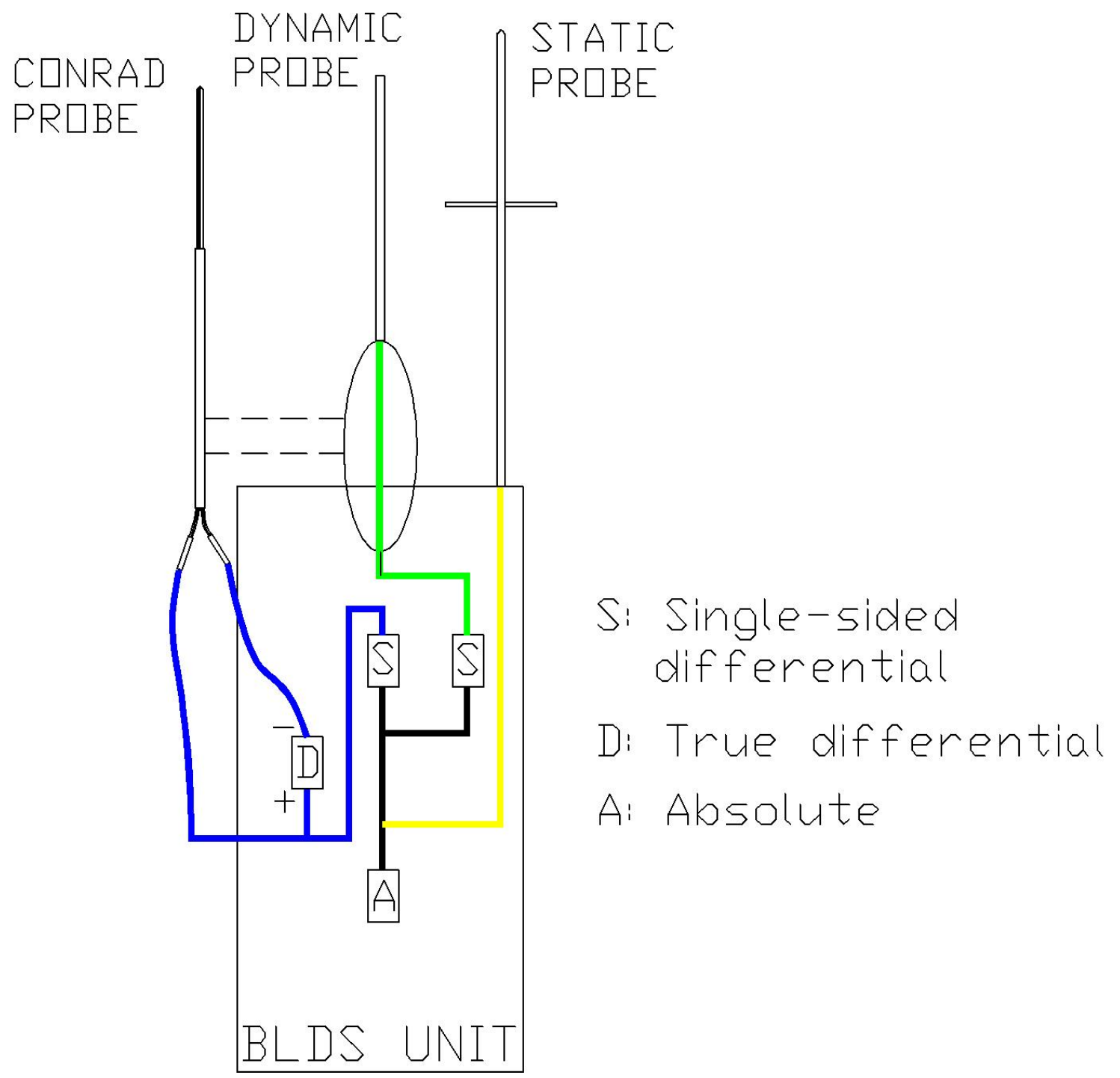

Figure 3.1 - Pressure sensor and probe layout for Conrad probe configuration of BLDS unit 


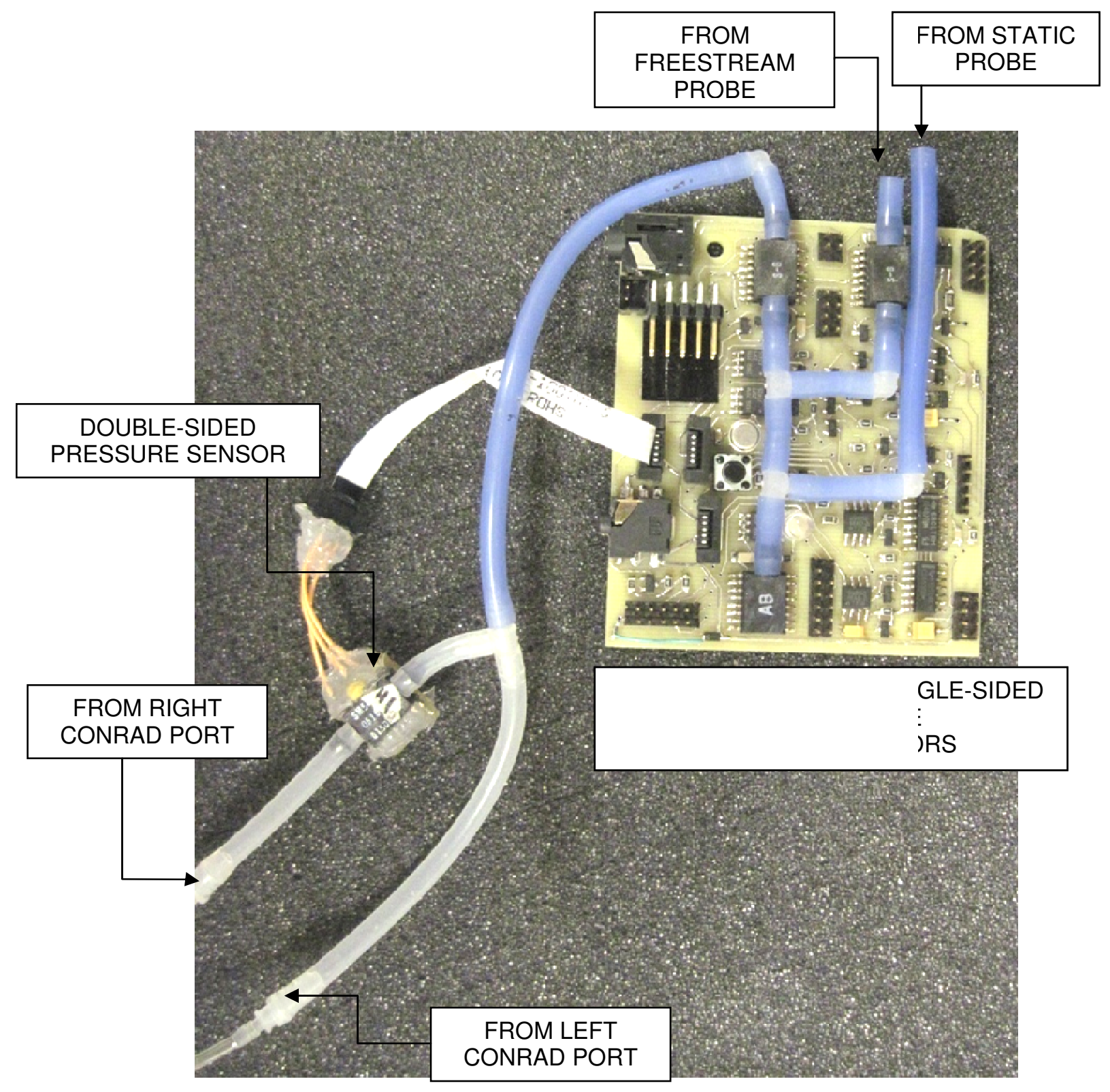

Figure 3.2 - Photograph of BLDS board with attached differential pressure sensor (TFX-11v2 removed) 
Figure 3.2 shows the pressure sensor configuration of the BLDS circuit board, with TFX controller removed, for use with the Conrad probe. Attachment of pressure probes to the BLDS stage assembly was typically done using a modified L-bracket with a close tolerance through hole in which probes were secured with a drop of glue. This setup meant that the probe and L-bracket were one assembly and could not be easily separated if probe modification was needed. An improved means of attachment was developed in which an Lbracket with a collet-type head secured probes in place.

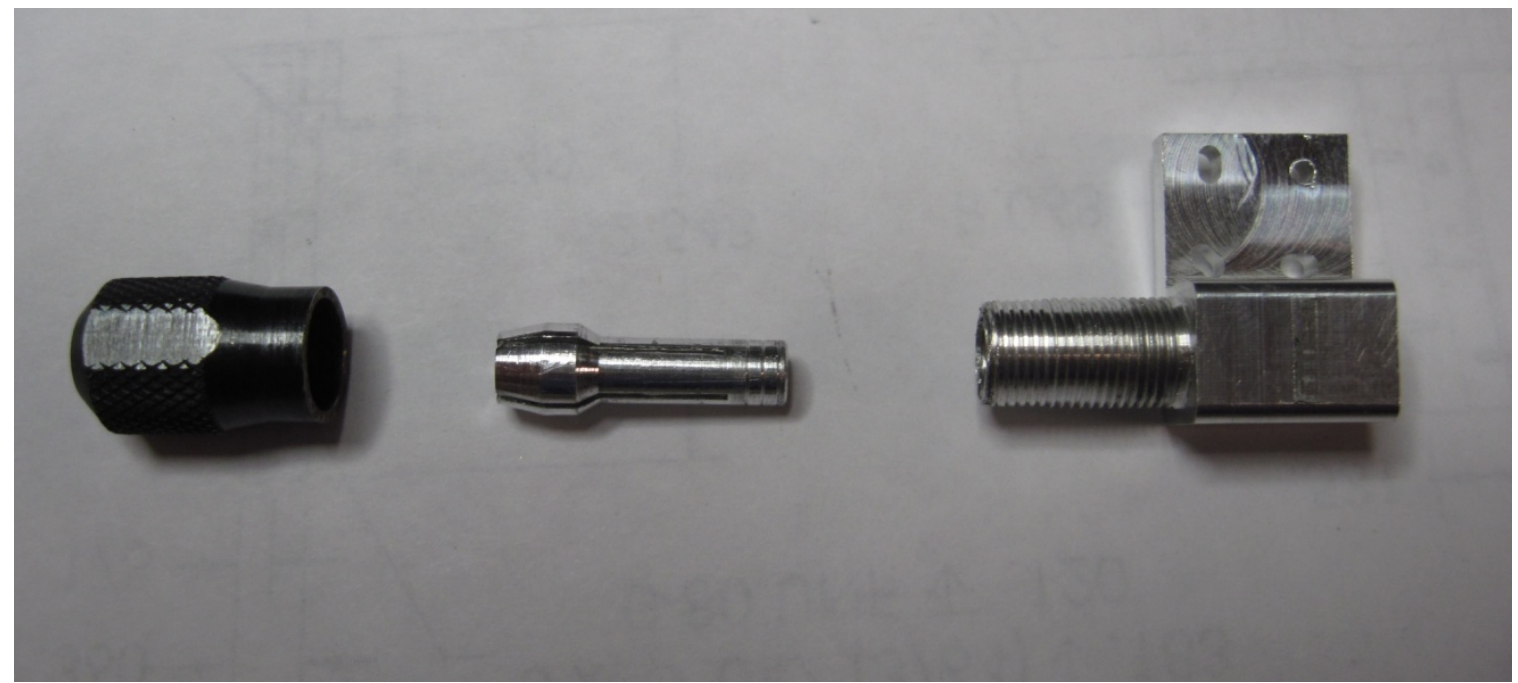

\section{Figure 3.3 - Collet-head L-Bracket for attachment of probes to BLDS unit}

With the additional pressure sensor added to the BLDS as well as the addition of the collet-head L-bracket for probe attachment, the Conrad probe was fully integrated into the hardware of the BLDS unit with the software integration still needing to be addressed.

The software for the BLDS unit is controlled with the use of a commercial dual microcontroller, the TFX-11v2 from Onset Computer Corp. The DAQ board is controlled through the use of a proprietary software development environment 
and a BASIC-like programming language called TFBASIC. Programs are uploaded to the $128 \mathrm{~kb}$ of volatile memory on board the microcontroller and are executed to enable pressure measurements along with any peripheral hardware associated with a particular measurement scheme.

To utilize the Conrad probe on the BLDS unit, the main data collection program was modified to include an additional menu option, data collection subroutine, and pressure sensor input. The additional menu option enabled data acquisition to take place with the Conrad probe. The added Conrad probe data collection subroutine included code for data to be taken from the three existing pressure sensors along with the added input for the additional double sided differential pressure sensor. The program was tested and debugged on the bench top and in the wind tunnel using the prototype 0.064" Conrad probe. The overall program flow for the BLDS unit is shown below in Figure 3.4; where the main changes were made in the "Startup Initialization" and "Acquire Data Point" portions of the program. A complete program listing is given in Appendix A. 


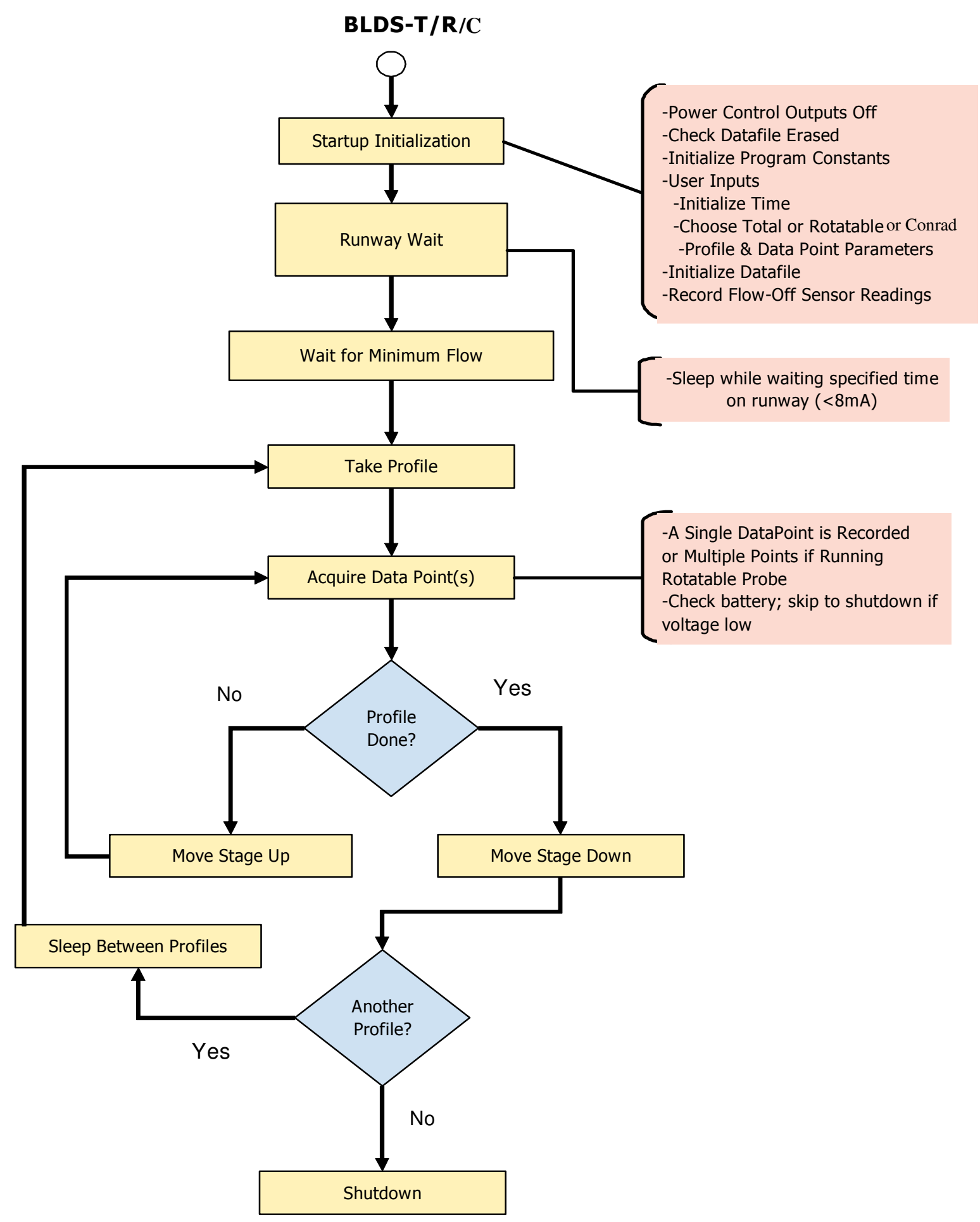

Figure 3.4 - Program flow for BLDS unit 
After adapting the BLDS hardware to work with the Conrad probe and updating the software the calibration from Chapter 2 could be implemented. The pressure sensor measurements needed were defined as follows from the setup seen above:

$$
\begin{gathered}
P_{L}=P_{\text {Left tube }}-P_{\text {static }} \\
P_{L-R}=P_{\text {left tube }}-P_{\text {right tube }} \\
q_{R E F}=P_{\text {reference }}=P_{\text {rynamic }} \quad \text { torence } \\
\text { total }
\end{gathered}
$$

Where, $P_{L}$ represents the pressure from the left side tube designated during calibration. $P_{L-R}$ denotes the pressure difference between the designated left and right tubes. $\mathrm{q}_{\mathrm{REF}}$ denotes the reference dynamic pressure obtained from a total pressure probe located outside the boundary layer. $P_{\text {static }}$ refers to the pressure recorded from the static probe on the surface [31]. To obtain the first computed quantity, the yaw angle $\beta$, the measured pressures obtained from $P_{L}$ and $P_{L-R}$ are used to calculate the following quantities:

$$
\begin{aligned}
& P_{A V G}=P_{L}-\frac{1}{2} P_{L-R} \\
& C_{P \Delta}=\frac{P_{L-R}}{P_{A V G}}=g(\beta)
\end{aligned}
$$

Combining the $P_{A V G}$ with the equation for $C_{P}$ and the calibration function, $g(\beta)$, which was found above in Chapter 2 , the yaw angle $\beta$ for a data point is found through inversion of the calibration. The next computed quantity of interest is the non-dimensional dynamic pressure, $\frac{q}{q_{R E F}}$. This is found with the second calibration relation, $f(\beta)$, and the values of $P_{A V G}$ and $q_{R E F}$ : 


$$
\begin{gathered}
\frac{P_{A V G}}{q_{R E F}}=\frac{P_{A V G}}{q} \frac{q}{q_{R E F}} \\
\frac{P_{A V G}}{q}=C_{P A V G}=f(\beta) \\
\frac{q}{q_{R E F}}=\frac{1}{f(\beta)} \frac{P_{A V G}}{q_{R E F}}
\end{gathered}
$$

where the non-dimensional dynamic pressure $\frac{q}{q_{R E F}}$ can be solved for by using the known $P_{A V G}$, the known value of the calibration function $f(\beta)$, and the final measured quantity $\mathrm{q}_{\mathrm{REF}}$. From here the non-dimensional velocity magnitude $\frac{U}{U_{R E F}}$ can be found by taking the root of the non-dimensional dynamic pressure.

The successful integration of the Conrad probe into the BLDS system meant that testing could now be done to validate the calibration technique and prepare the Conrad probe for its eventual use in measuring laminar boundary layers on swept wing aircraft. 


\section{WIND TUNNEL TESTING}

After manufacturing the Conrad probe, developing and applying a calibration scheme, and adapting the probe for use on the BLDS system, testing was done to determine if the prototype probe would be usable in field conditions. This testing had three parts; first, the prototype 0.064 " diameter probe was tested inside of a thick turbulent boundary layer on the bottom surface of the 2'x2' wind tunnel. Next, when results were obtained, another smaller diameter probe was tested in a thin laminar boundary layer developed an elliptical nose flat plate in the wind tunnel. Finally, the smaller diameter probe was tested in another thin boundary layer on a swept flat plate.

Table 4.1 - Test Matrix for Wind Tunnel Evaluations

\begin{tabular}{|c|c|c|c|c|}
\hline Test & $\begin{array}{c}\text { Probes } \\
\text { Used }\end{array}$ & Location & $\begin{array}{c}\text { Reynolds } \\
\text { Number }\end{array}$ & $\begin{array}{c}\text { Mach } \\
\text { Number }\end{array}$ \\
\hline $\begin{array}{c}\text { Wind Tunnel } \\
\text { Floor }\end{array}$ & $\begin{array}{c}\text { Conrad \& } \\
\text { Total }\end{array}$ & $\begin{array}{c}33 \text { inches aft contraction } \\
\text { exit }\end{array}$ & $2.95 \mathrm{e} 6$ & 0.14 \\
\hline $\begin{array}{c}\text { Unswept Flat } \\
\text { Plate }\end{array}$ & $\begin{array}{c}\text { Conrad, } \\
\text { Total, \& } \\
\text { Rotatable }\end{array}$ & $\begin{array}{c}12 \text { inches aft of leading } \\
\text { edge }\end{array}$ & $9.84 \mathrm{e} 5$ & 0.14 \\
\hline $\begin{array}{c}\text { Swept Flat } \\
\text { Plate }\end{array}$ & $\begin{array}{c}\text { Conrad, } \\
\text { Total, \& } \\
\text { Rotatable }\end{array}$ & $\begin{array}{r}\text { 8 inches aft of leading } \\
\text { edge }\end{array}$ & $6.56 \mathrm{e} 5$ & 0.14 \\
\hline
\end{tabular}

After obtaining favorable results in the wind tunnel the small probe was used as part of a flight test regimen for Northrop Grumman Corporation. Wind tunnel testing was carried out with the use of a 2' square wind tunnel section, 
capable of attaining flow velocities of $110 \mathrm{mph}$ corresponding to a nominal maximum dynamic pressure of $30 \mathrm{psf}$.

The BLDS unit was placed in the wind tunnel in various configurations, all of which use the conventions shown in Figure 4.1; where " $x$ " is defined as the distance from either the start of the test section (for the initial turbulent measurement) or leading edge (for the laminar measurements on the plates), $\alpha$ is the angle of pitch of the probe (typically $3-5^{\circ}$ depending upon which probe is used) and " $y$ " is the vertical distance away from the surface.

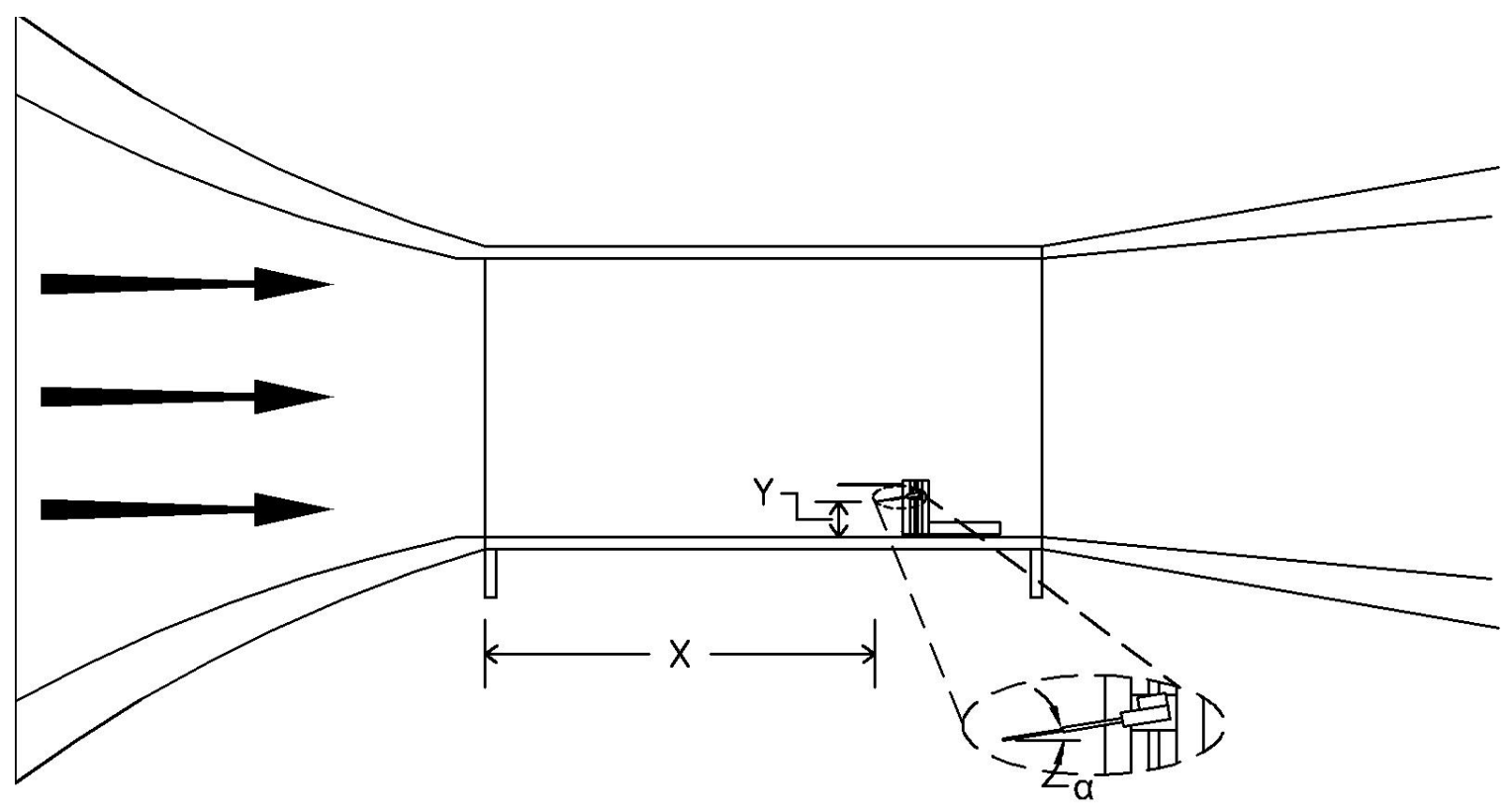

Figure 4.1 - Diagram of BLDS in 2' x 2' wind tunnel

The vertical distance " $y$ " is found using corrected data from the encoder on the BLDSs traversing stage. The correction to this vertical data is needed due to probe tips flexing when they contact the surface at the point where the stage is at its lowest point. The flexing of the probe tips causes the probe to continue to touch the surface as the stage encoder counts increase, causing a discrepancy 
between the location of the measured value and the recorded vertical location. To correct this, the encoder data is converted from counts to a linear measurement then analyzed, along with the pressure data, to the find the location where the probe leaves the surface or the "lift-off" point. This lift-off distance is subtracted from the stage position and with the result then added to the height of the probe tip centerline. Figure 4.2 shows an example of the "y" correction, where $d$ represents the stage position, $d_{\text {liftoff }}$ is the lift-off distance, and $D$ is the probe diameter.

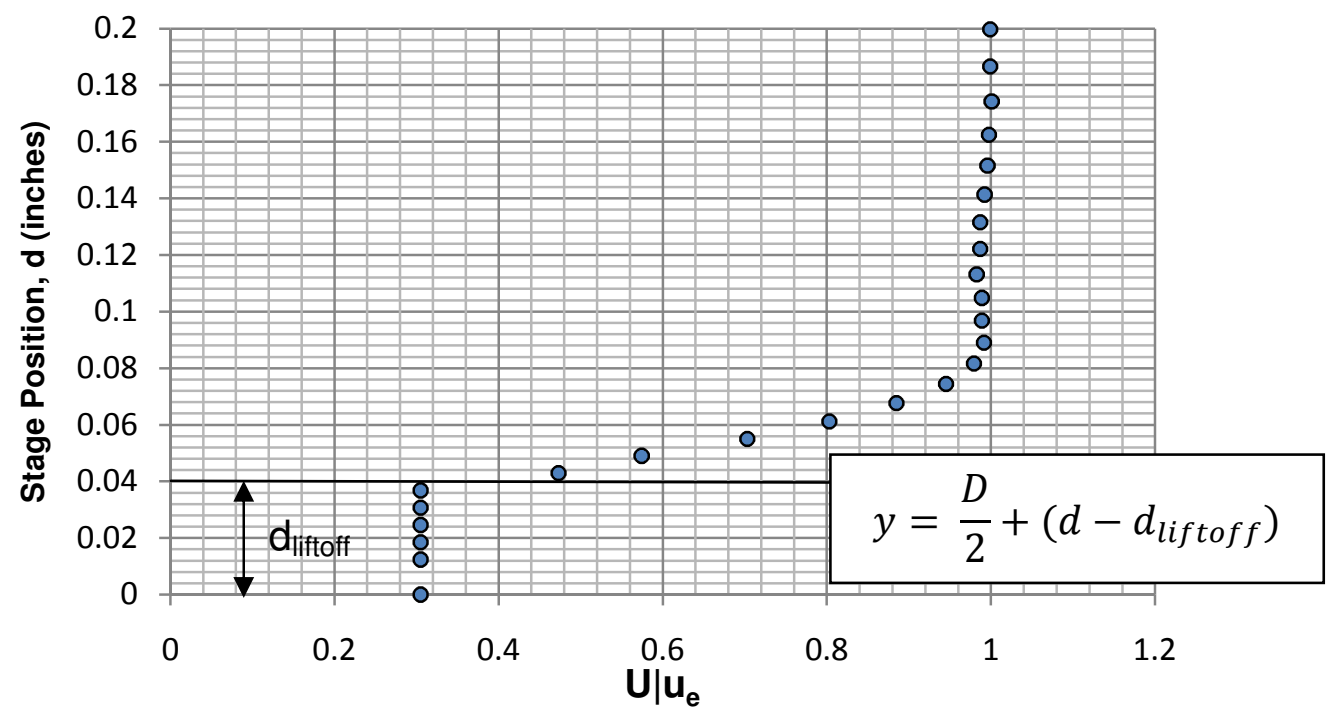

Figure 4.2 - Example of calculation of vertical distance " $y$ "

For the initial test, the prototype 0.064 " probe was adapted for use on the BLDS unit and placed in the wind tunnel at a location 33 inches from the contraction exit. Testing was done in a turbulent state $(\operatorname{Re} \sim 1 \mathrm{e} 7)$ at maximum dynamic pressure to determine if the probe could resolve yaw angle and flow velocity. Figure 4.3 shows the BLDS in the wind tunnel. 


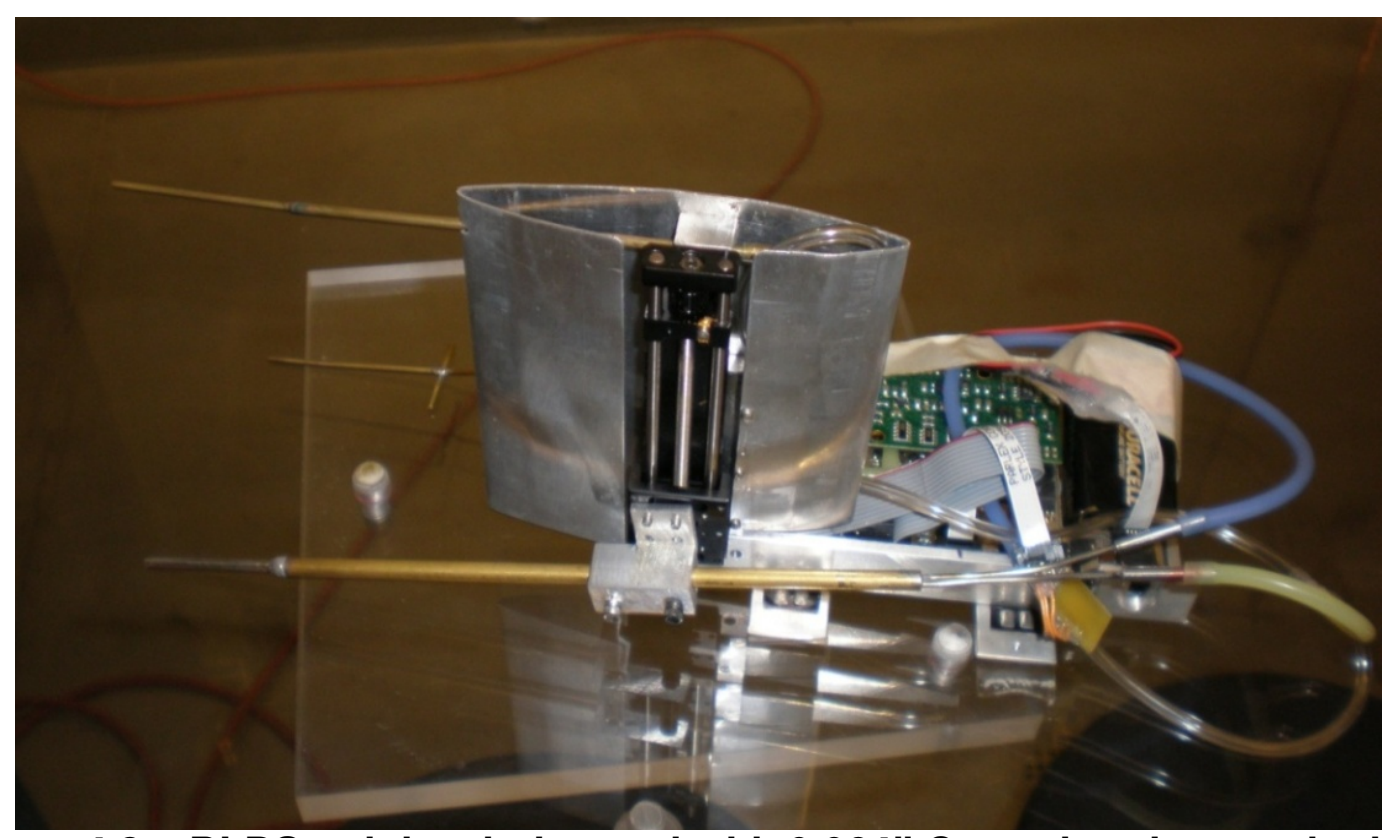

Figure 4.3 - BLDS unit in wind tunnel with 0.064" Conrad probe attached

Results from the wind tunnel floor are shown in Figures 4.4 and 4.5 below where the Conrad probe was used to obtain and a boundary layer profile and a yaw measurement. Boundary layer measurements were non-dimensionalized to aid in comparisons between probes. The parameter $u_{e}$ was defined as the value of the velocity measured by the probe in the free-stream. The non-dimensional boundary layer profile was compared to total probe data taken at the same location on the bottom surface on the wind tunnel section. 


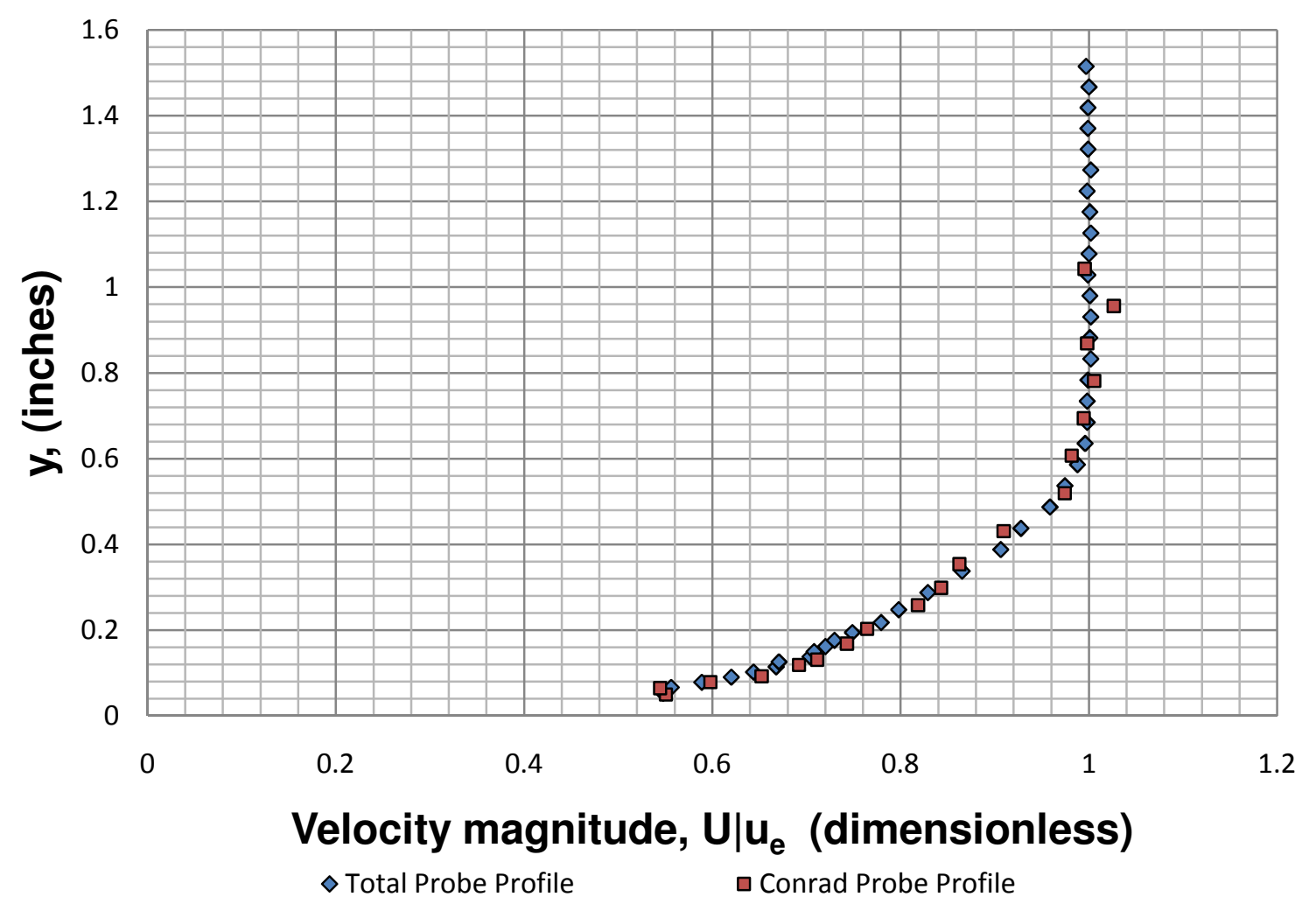

Figure 4.4 - U| $\mathrm{u}_{\mathrm{e}}$ vs. $y$ for total and Conrad probes in 2'x2' wind tunnel on bottom surface, 33 inches from contraction exit

The initial data showed that the Conrad probe and total probe profiles both measured a turbulent boundary layer 0.6 inches thick. The agreement between these results indicated that the prototype Conrad probe was able to measure the velocity profile of a boundary layer. As shown in Figure 4.3, the BLDS unit was yawed in order to obtain a reading from the probe that could be compared to a measured angle. In this case the angle of the probe on the BLDS was measured with respect to the centerline of the wind tunnel. Figure 4.5 reveals that when yawed at a 10.4 degree angle, the prototype Conrad probe could successfully measure the yaw, indicating that the calibration was working and that further laminar tests with the smaller probe could be performed. 


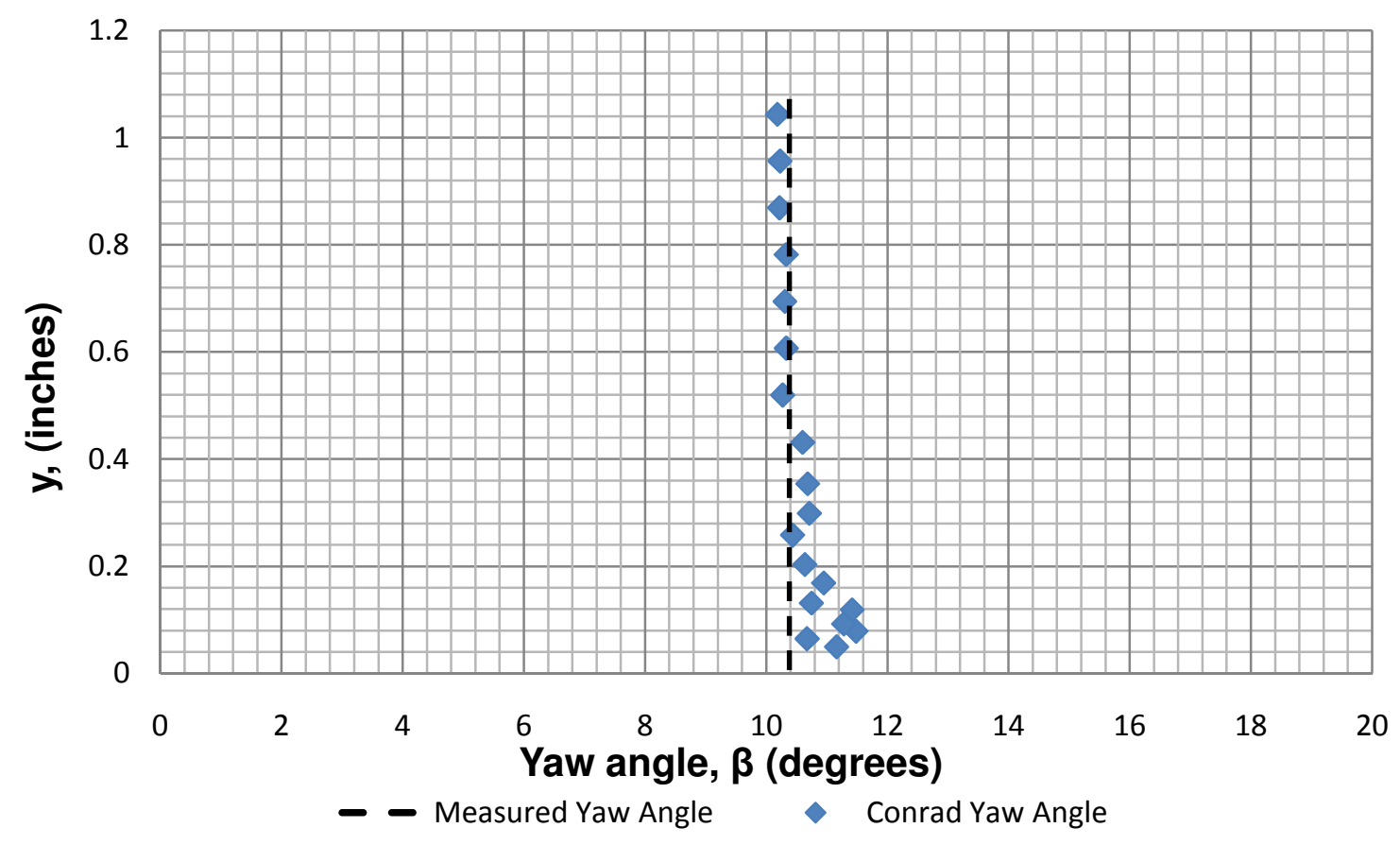

Figure 4.5 - Yaw angle, $\beta$ vs. height for BLDS unit in 2'x2' wind tunnel with 0.064 " Conrad probe attached and yawed at 10.4 degrees

As noted above, these results were obtained at a dynamic pressure of 30 psf, the nominal operational maximum of the wind tunnel. Measurements obtained at this pressure only utilize $15 \%$ of the available range of the BLDS pressure sensors; thus increasing uncertainty as discussed in Chapter 2. The results obtained from these preliminary tests showed values well within the uncertainty ranges found for wind tunnel testing, thus the calibration was found to be valid and the prototype Conrad probe successfully resolved both flow direction and magnitude of a turbulent boundary layer developed on the bottom surface of the wind tunnel. Due to its large diameter, the prototype Conrad probe did not have a small enough measurement volume to measure the thin laminar boundary layers found in flight tests, therefore another smaller probe was manufactured 
and tested to measure these thin boundary layers. A 0.020" diameter Conrad probe was manufactured, calibrated, and readied for testing in the wind tunnel. Since laminar boundary layers were needed for the next set of tests, an elliptical nose flat plate was used. The elliptical leading edge allowed oncoming flow to accelerate slowly around the leading edge of the plate, forming conditions conducive to the development of laminar boundary layers on its surface. This plate was placed inside the test section and the BLDS unit was installed at a distance of $1 \mathrm{ft}$ from the leading edge $(\mathrm{x} \mid \mathrm{c}$ of 0.25$)$ using $3 \mathrm{M}$ double-sided pressure cured adhesive and a spring pressure loading fixture. The predicted boundary layer height at this point was estimated to be 0.06 " using the Blasius solution for laminar flow over a flat plate [26]. A set of tests at maximum wind tunnel dynamic pressure were performed with the BLDS unit yawed at a measured angle of 9.7 degrees (clockwise) from the main flow direction. Figure 4.6 shows the flat plate along with the $2^{\prime} \times 2$ ' wind tunnel test section. Figures 4.7 and 4.8 depict the BLDS unit yawed on the plate and installed in the wind tunnel in its flight configuration with a surface static probe mounted separately on the surface of the flat plate. 


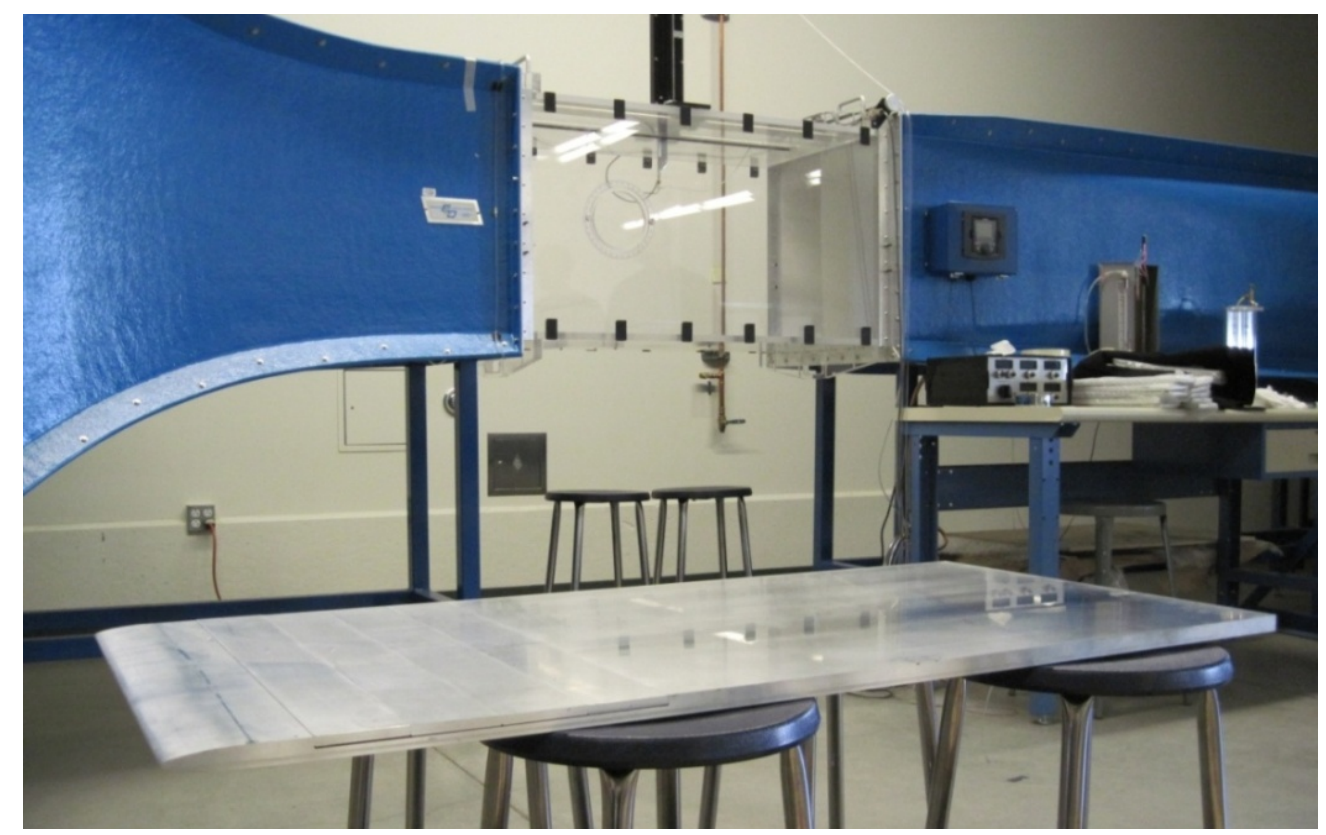

Figure 4.6 - 2' wind tunnel test section and elliptical nose flat plate

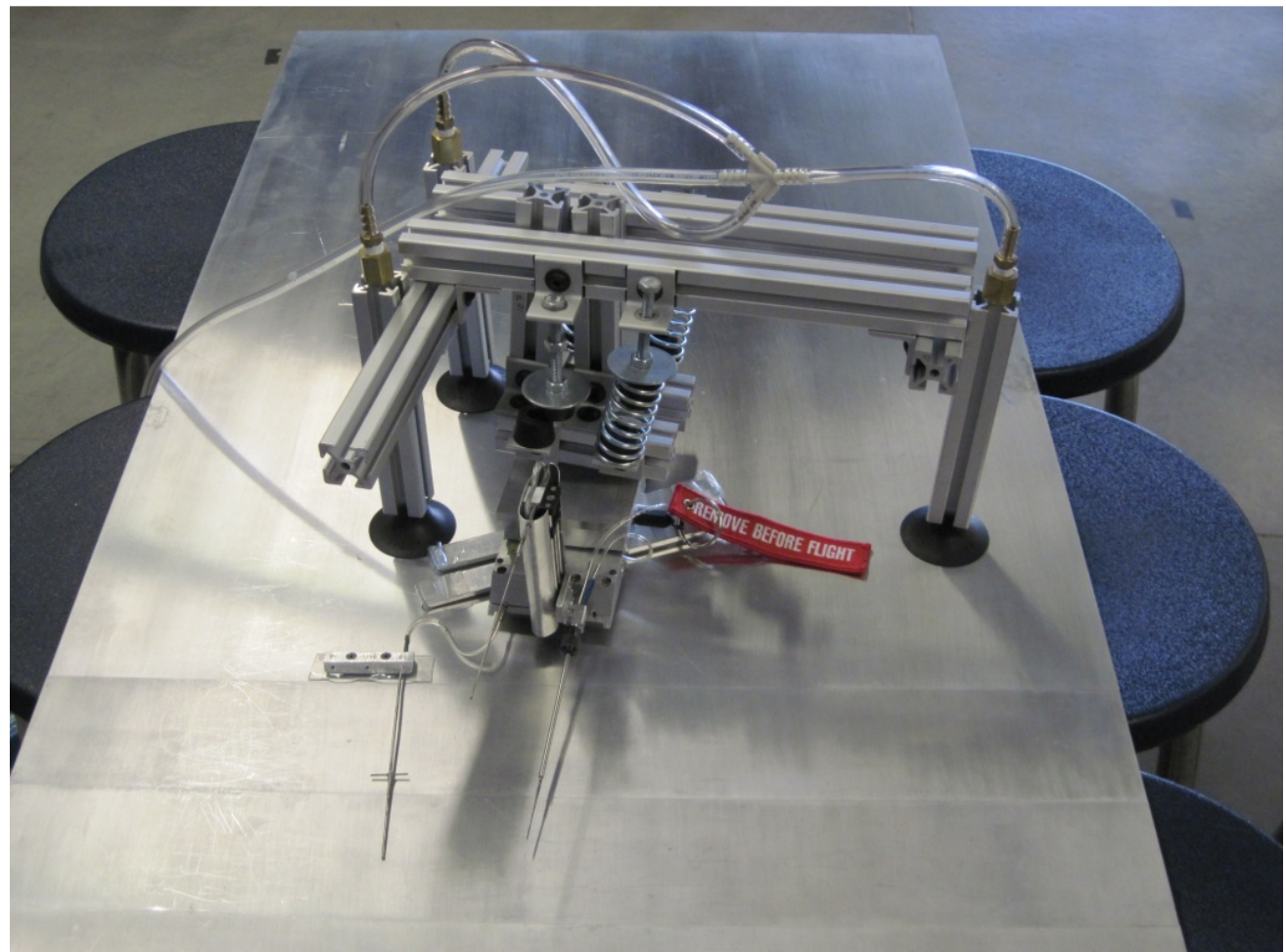

Figure 4.7 - BLDS with Conrad probe being installed on flat plate with spring load fixture 


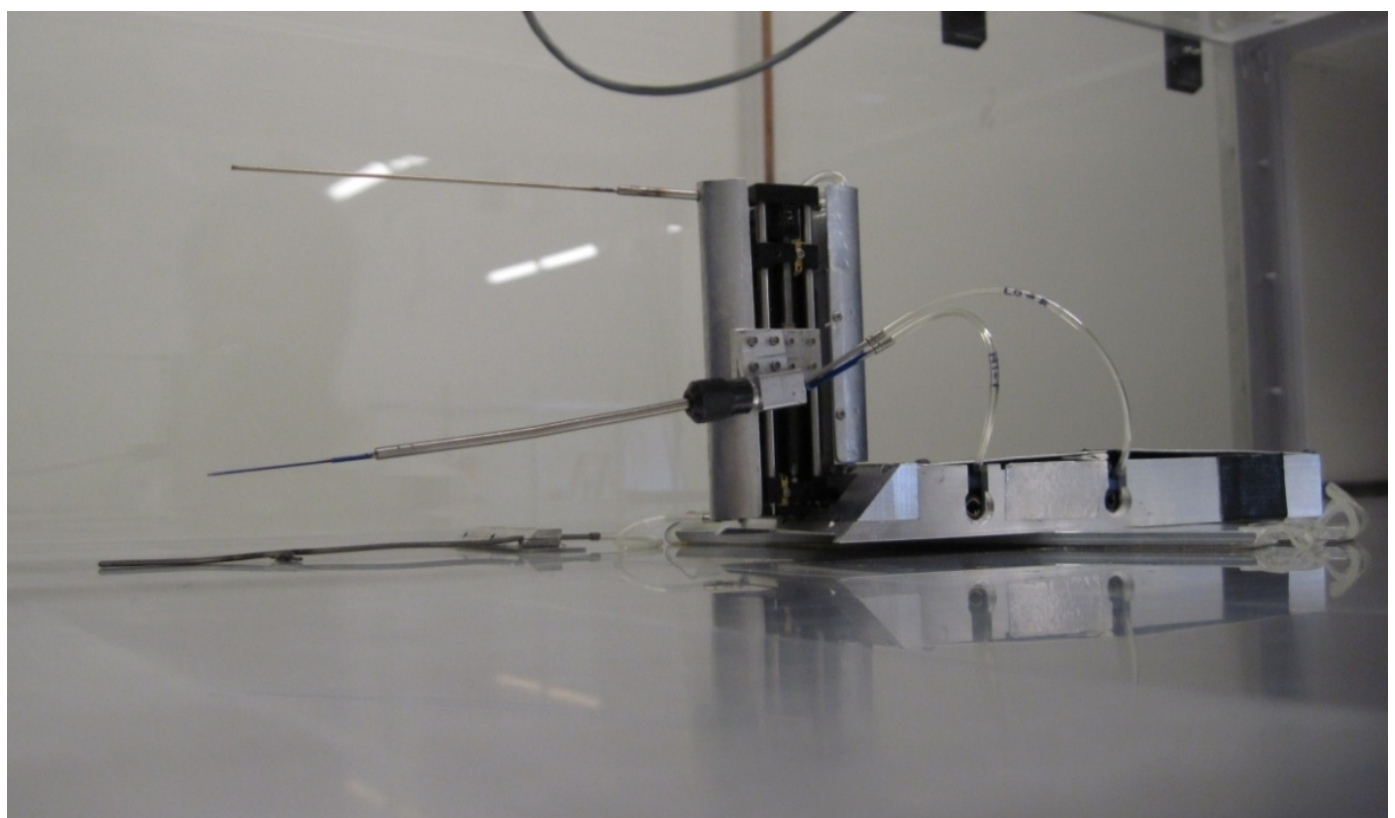

Figure 4.8 - 2' wind tunnel with BLDS-C installed on flat plate

The BLDS unit was run in all three of its main flight configurations including the total configuration for velocity magnitude measurements, the rotatable configuration for flow pitch angles, flow yaw angles, and velocity magnitude measurements, and the Conrad configuration for flow yaw angles and velocity magnitude measurement. From this data three comparisons of the Conrad probe's performance were found; the first was direct comparison of the velocity magnitude measurements from the Conrad, rotatable, and total probes. Each set of probe data contains ten profiles taken at a constant dynamic pressure. Figure 4.9 shows a comparison of the Conrad, total, and rotatable velocity profiles. 


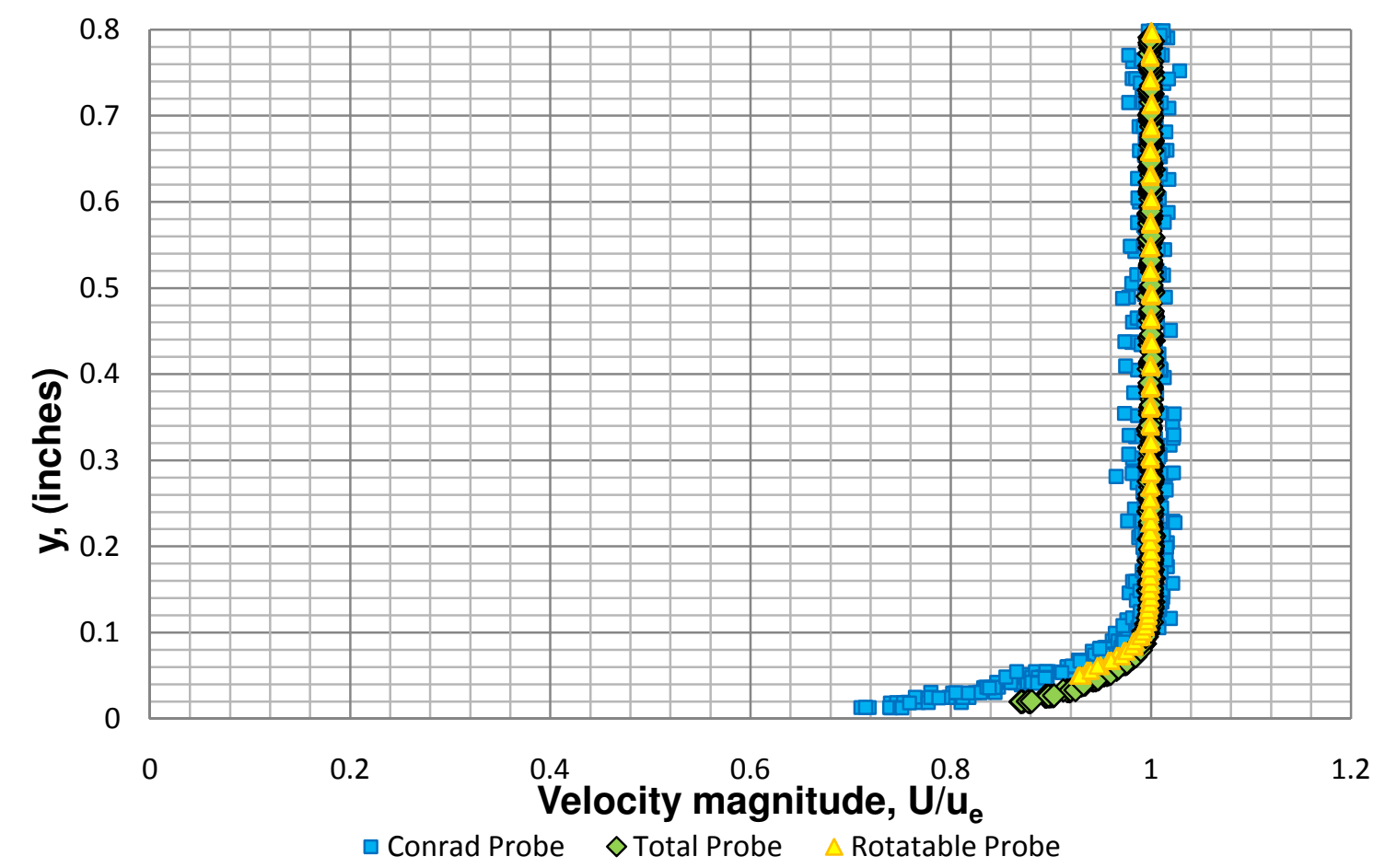

Figure 4.9- Boundary layer profiles of velocity magnitude on elliptical nose flat plate at $\mathrm{x} / \mathrm{c}$ of 0.25 for total, rotatable, and Conrad probes

Once again, the boundary layer measurements were non-dimensionalized to aid in probe comparisons. The parameter $u_{e}$ was defined as the velocity measured by the each probe in the free-stream. The velocity data shows the presence of a laminar boundary layer, illustrated by both the nearly linear profile shape and the thinness of the boundary layer. Estimations of turbulent boundary layer thickness give the turbulent boundary layer a height of $\sim 0.25 "$, which is far above the 0.08 " thickness that was measured by all three probes. The agreement of the results with the estimated laminar boundary layer thickness of 0.06 " made it clear that a laminar profile was obtained. The total probe data represents a baseline case as this type of probe has been rigorously tested with the BLDS unit. The Conrad probe velocity magnitude data shows good 
agreement to within $2 \%$ of the baseline total probe data; there is some scatter in the Conrad data, which is discussed at the end of this chapter. In addition to the Conrad probe results, the rotatable probe data matches the shape and thickness of the measured total probe boundary layers for the Conrad and total probes. In an overall sense, this measurement and its subsequent agreement with the analytical solution clearly show the potential of the Conrad probe to measure velocity profiles on natural laminar flow airfoils.

The second comparison shows the in-plane velocity component, $w \mid u_{e}$, measured by both the rotatable and Conrad probes, perpendicular to the probe axes. Due to yawed orientation of the probe, this value was expected to change as the probe moved vertically through the boundary layer and then become constant in the free-stream. The measurements from the Conrad and rotatable probes are shown in Figure 4.10 below.

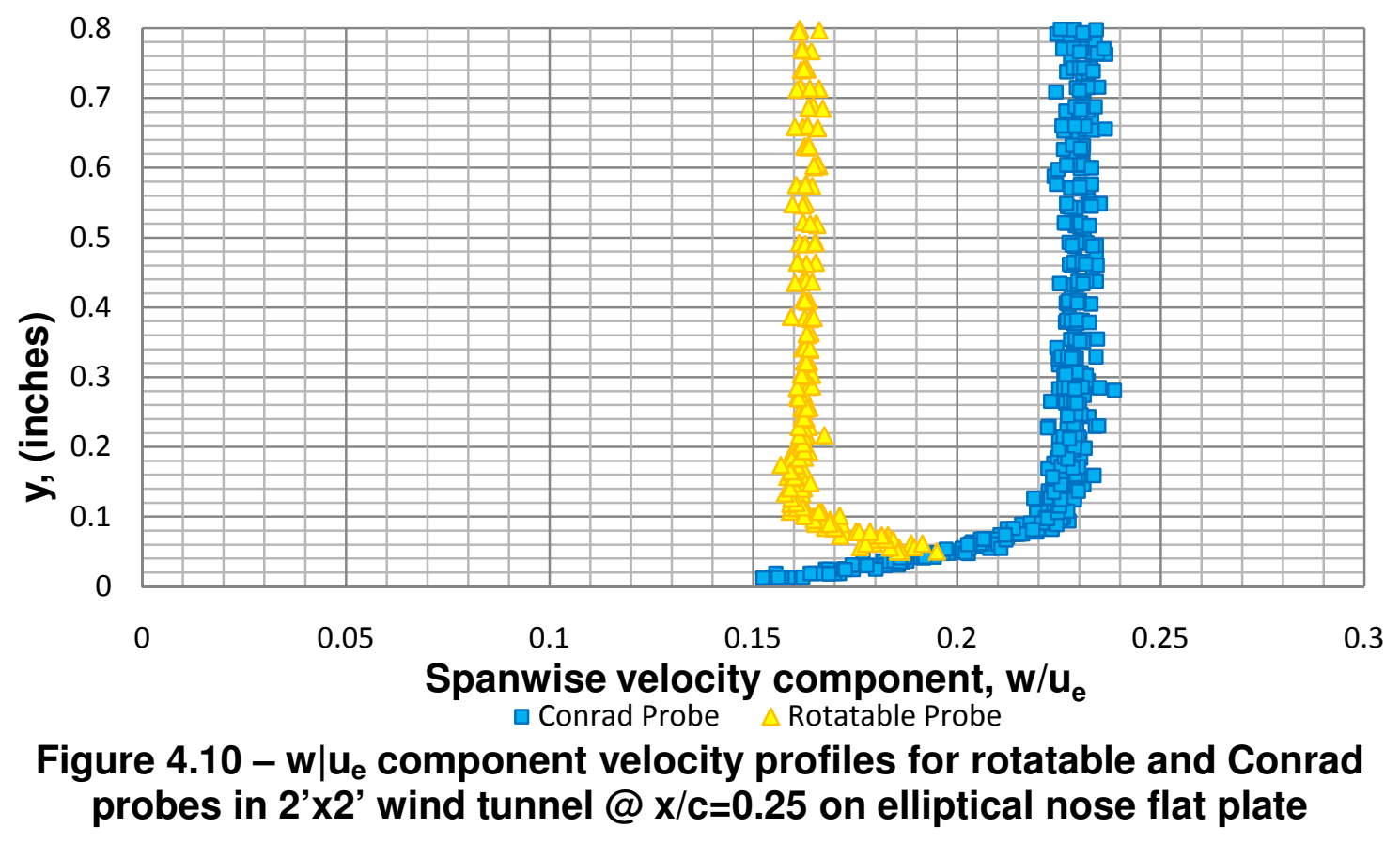


The $w \mid u_{e}$ component plot for the Conrad probe shows a familiar laminar velocity profile with a boundary layer thickness the same as the overall measurement. The measurement changes with height, as predicted, and becomes constant as the probe leaves the boundary layer. The rotatable measures the constant free-stream component, but shows variation at the surface. Such variation is shown to occur in experiments where measurements are made within two probe diameters of the surface [16]. Therefore it is assumed that the large probe diameter of the rotatable probe used and thinness of the boundary layer at this chord-wise location are the causes of skewing near the surface.

The final comparison, in Figure 4.10, presents the yaw angle measurements taken by both probes. The yaw angle on the plate was predicted to remain constant the boundary layer.

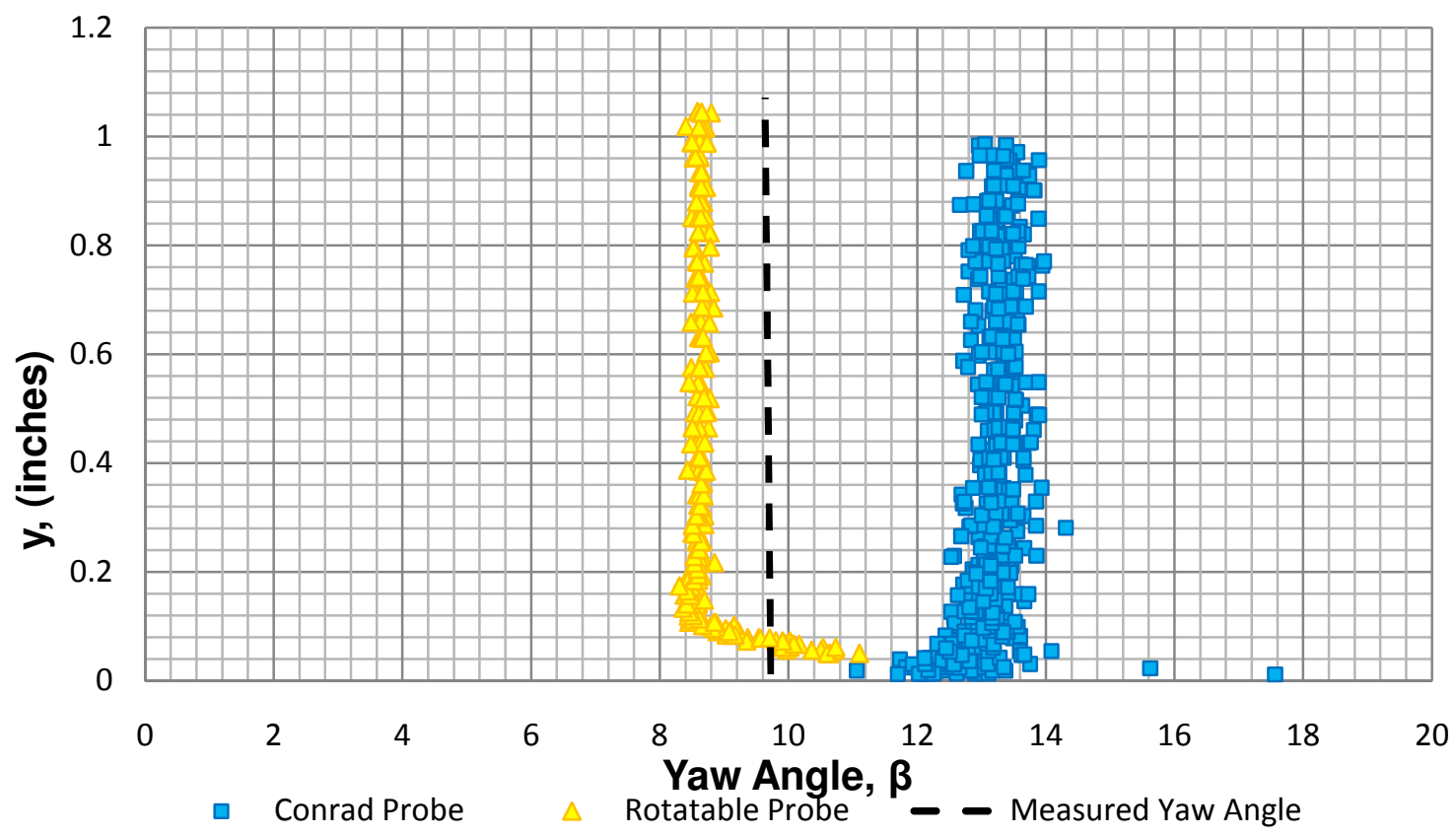

Figure 4.11 - Yaw angle, $\beta$ vs. height for rotatable and Conrad probes in 2'x2' wind tunnel @ x/c=0.25 on elliptical nose flat plate 
The same skewing effect close to the surface is seen again in the rotatable probe results, indicative of surface effects for the large diameter rotatable probe when within two diameters of the surface. Aside from the skewing, the rotatable data appears to agree within one degree of the measured yaw angle of the probe and also shows a constant yaw angle in the free-stream as expected. The Conrad probe data shows a four degree difference from the rotatable and measured yaw angle data. This difference can be accounted for by the probe manufacturing inconsistencies that brought about the use of the angular offset term in the calibration equations from Chapter 2. Given that flow angle can never be measured with perfect certainty with respect to a fixed coordinate system that is separate from that of a pressure probe, the trend exhibited by the data is of more use than the actual measured angle. The Conrad probe data shows a constant yaw angle as the probe traverses the boundary layer, agreeing with the predicted result. Additionally, there is also an amount of scatter exhibited by the yaw angle data; however the data is all within the uncertainty range of $\pm 1.25^{\circ}$.

The flat plate data demonstrates the ability of the Conrad probe to resolve flow velocity magnitude and in-plane components of flow in a boundary layer. This highlights its potential usefulness in applications such as swept wings, where in-plane velocity components need to be measured close to a surface. The next step was to determine if the Conrad probe could measure variation in the span-wise component of flow over a swept wing. The unswept flat plate used previously would not simulate a swept wing; therefore a new plate was designed 
to provide flow turning near to a leading edge. The new $30^{\circ}$ swept leading edge plate was designed to fit into the 2'x2' wind tunnel test section used previously. To get the right surface finish for development of laminar boundary layers, Blanchard ground aluminum tool plate was used to construct the plate. To get a slow acceleration of flow from the leading edge, a lengthened super-ellipse identical to the nose of the unswept flat plate was used. The final design for the plate specified a $1 / 2$ " thick plate with a 33 " chord, 23.5 " width, and a leading edge radius of $3 \mathrm{~mm}$. Additionally, the plate was designed as two pieces, an elliptical nose piece and a main plate body piece, for ease of manufacture.

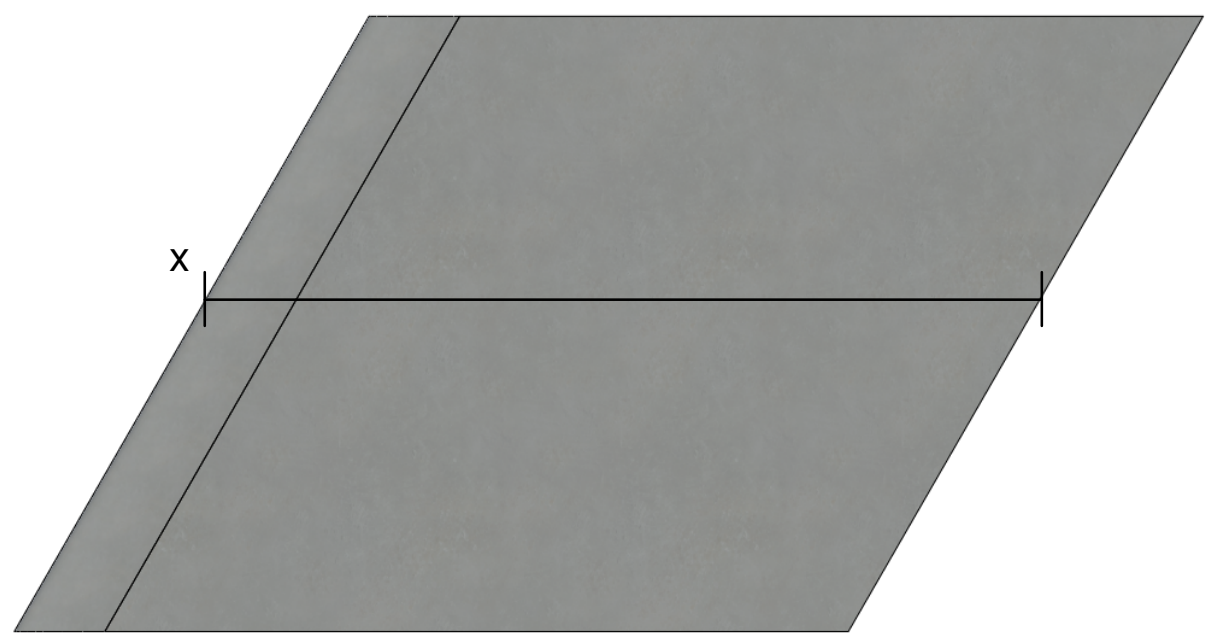

Figure 4.12 - Three-dimensional model of swept leading edge ellipticalnose flat plate

With a preliminary model developed for the swept flat plate, the amount of expected secondary flow in the boundary layer had to be estimated to ensure that appreciable measurements could be taken. No exact solution for a swept flat plate was found, however an exact method for obtaining the span-wise 
component of a three-dimensional boundary layer over an infinite yawed cylinder was previously explored by W.R. Sears in a 1964 paper [32]. Sears' solution reduces the three-dimensional Navier-Stokes equations with the result being a form of the equations that demonstrates the so-called "independence" principle, where the stream-wise velocity component can be calculated separately from the span-wise component. The resulting equations are then simplified from partial differential equations to sets of linear ordinary differential equations through use of a similarity transformation [32]. These sets of ODEs are subsequently solved in a collection of papers on laminar boundary layers compiled by L. Rosenhead [33]. Using this solution the amount of flow angle change with vertical height change was computed for various distances away from the leading edge. A full calculation is included in Appendix $C$, the results of which showed the presence of secondary flow in the boundary layer over the swept flat plate. The analytical solution was found to be valid to a point approximately two millimeters aft of the leading edge with 12 degrees of flow angle change occurring. With the assumption that the pressure distribution around the plate stayed constant, the angular change in the flow was assumed to continue downstream before being damped out by the free-stream velocity component. The presence of secondary flow on the plate made it a valid tool for the measurement of a three-dimensional boundary layer. Upon construction of the plate it was subsequently installed in the wind tunnel, in the same fashion as the unswept flat plate, and a series of tests were performed to both evaluate the flow around the plate and explore the capabilities of the Conrad probe in a laminar boundary layer. Before the first sets 
of tests could be performed, static pressure and skin friction surveys with the PTDS device and a probe foot were done. Figure 4.13 shows the PTDS installed upon the swept flat plate inside of the 2' $\times 2$ ' wind tunnel test section.

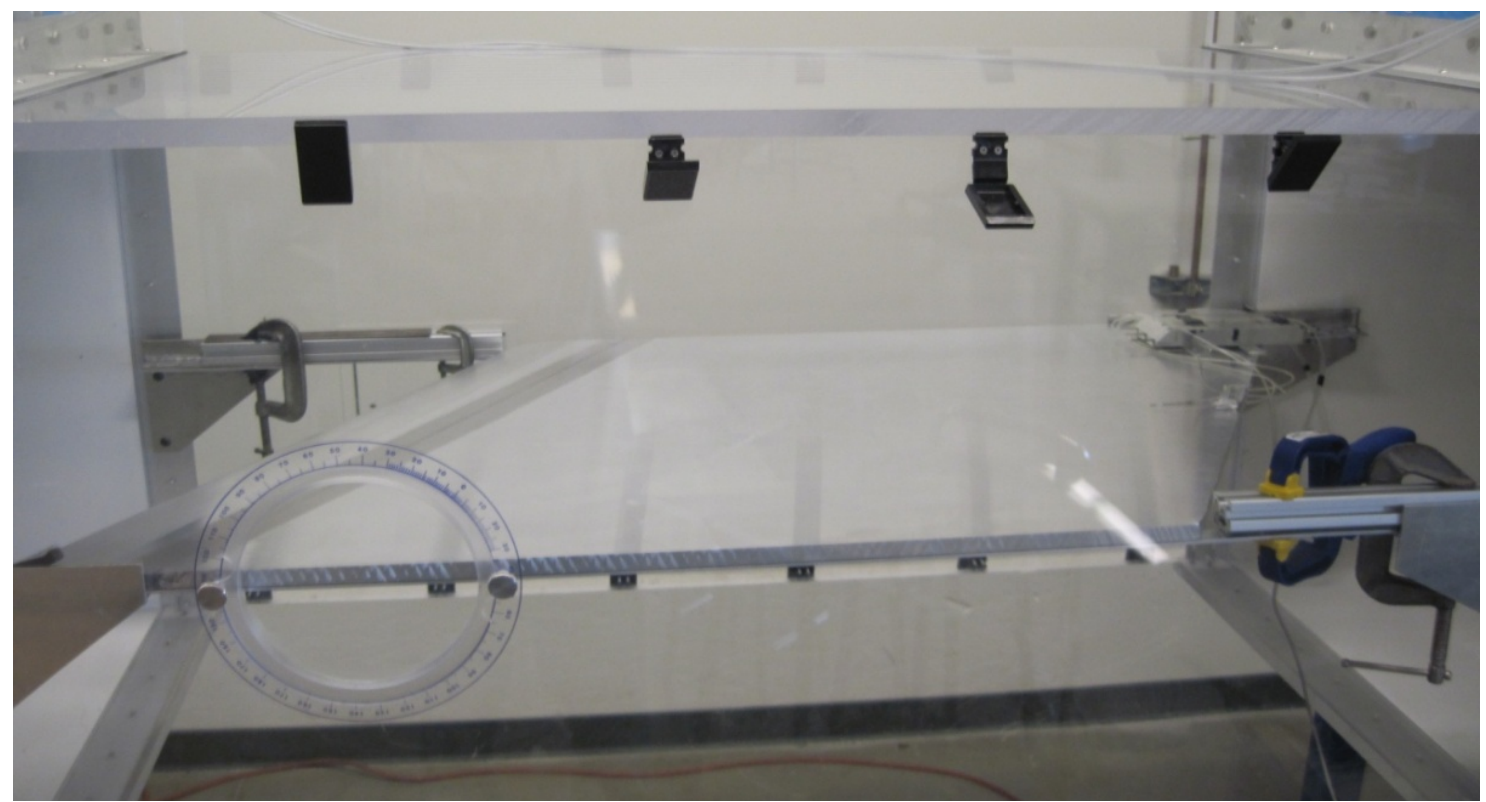

Figure 4.13 - PTDS unit installed with probe foot at $x / c=0.9$ on swept flat plate in 2' $\times 2$ ' wind tunnel test section

The device was activated and data was taken at the maximum wind tunnel speed of 110 miles per hour which corresponded to a dynamic pressure of $30 \mathrm{psf}$ and $\frac{R E}{L}=8.2 \mathrm{e} 4$ (per inch). Readings were taken at 29.5 inches from the leading edge of the plate and incremented forward by three inches per run. This process was repeated until a full survey along the centerline of the swept flat plate was completed. From the obtained data, a pressure distribution and skin friction distribution along the plate were obtained. Plots of these are shown below in Figures 4.12 and 4.13 . 


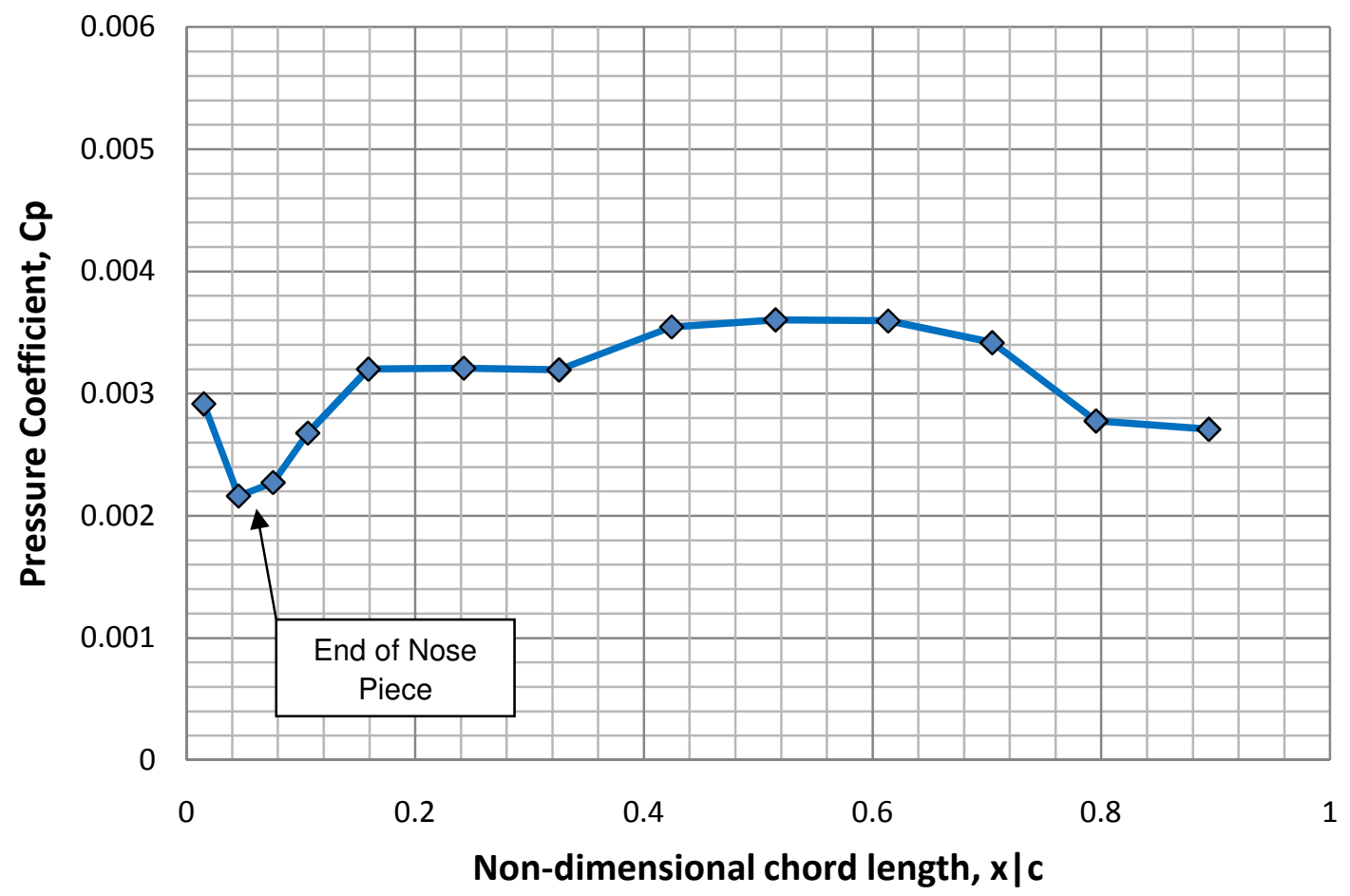

Figure 4.14 - $C_{P}$ vs. non-dimensional chord distance for swept flat plate in 2' $\times 2$ ' wind tunnel at $\mathrm{q}_{\mathrm{REF}}=30 \mathrm{psf}$

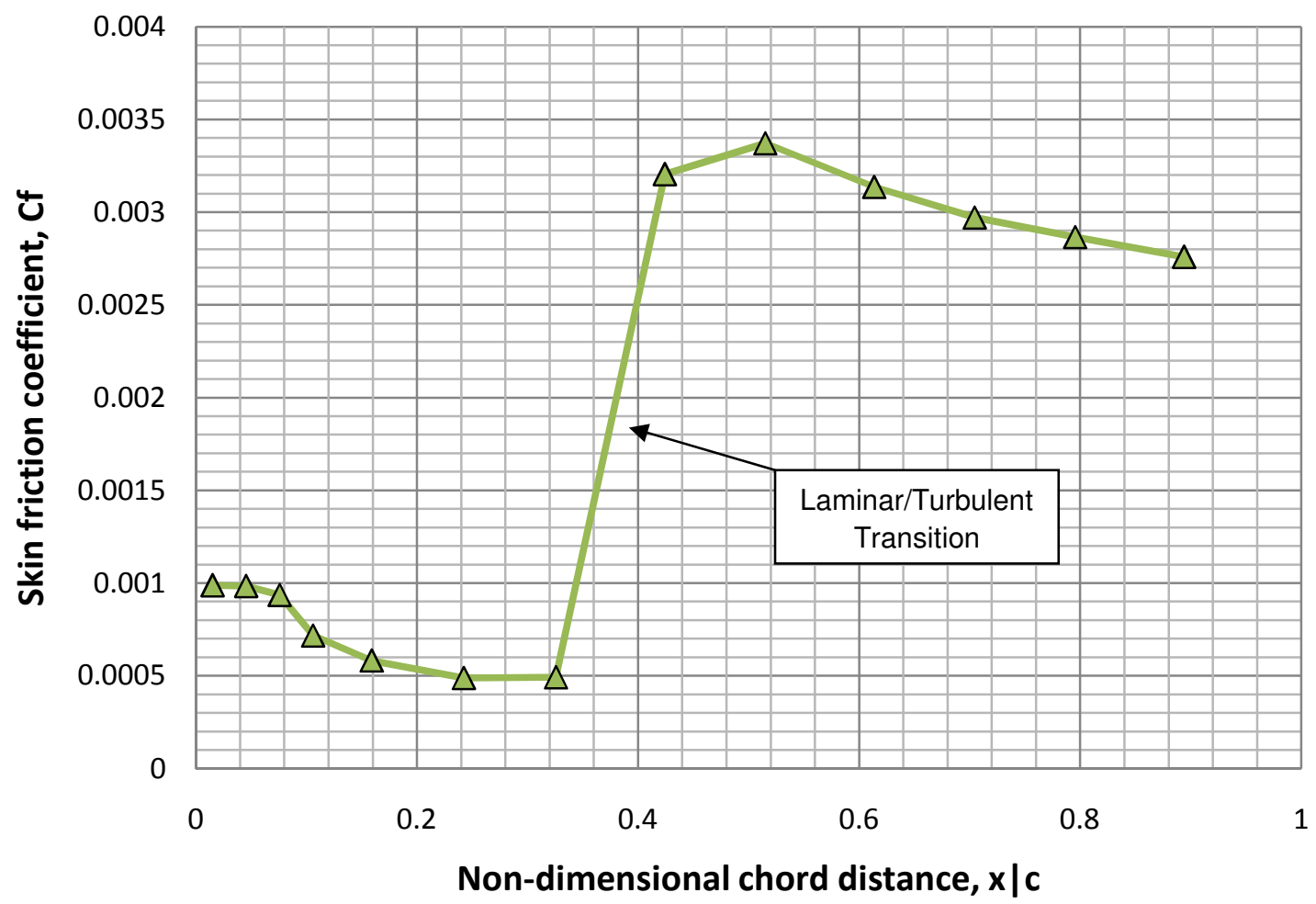

Figure 4.15 $-C_{f}$ vs. non-dimensional chord distance for swept flat plate in 2' $x$ 2' wind tunnel at $\mathrm{q}_{\mathrm{REF}}=30 \mathrm{psf}$ 
The pressure coefficient $C_{P}$ was defined as:

$$
C_{P}=\frac{P_{S}(x)-P_{R E F}}{q_{R E F}}
$$

where $\mathrm{P}_{S}(\mathrm{x})$ is the static pressure at any location along the plate, $\mathrm{P}_{\mathrm{REF}}$ is a reference static pressure obtained from the free-stream, and $\mathrm{q}_{\mathrm{REF}}$ is the reference dynamic pressure. The skin friction was defined as:

$$
C_{f}=\frac{\tau}{q_{R E F}}
$$

where $\mathrm{T}$ is the skin friction on the surface.

From these figures two conclusions were drawn; first, the $\mathrm{C}_{\mathrm{P}}(\mathrm{x})$ plot showed a negligible change in the pressure distribution along the plate, validating the previous assumption from the analytical Sears calculation. Secondly, the $\mathrm{C}_{\mathrm{f}}(\mathrm{x})$ plot showed a transition from laminar to turbulent flow on the plate surface to occur roughly 13 inches from the leading edge of the plate $(x \mid c=0.4)$. This transition point provided a basis for finding an optimal installation location for the BLDS to measure laminar boundary layers. Installation directly ahead of the transition point was problematic due to the small amount of secondary flow at the location. The Sears solution predicted the largest amount of secondary flow to occur within two millimeters of the leading edge, however due to the extreme thinness of the boundary layer at this location a compromise was made between this and the transition point. The $0.020 "(0.5 \mathrm{~mm})$ diameter Conrad probe was best used in a boundary layer that was at least two diameters thick, therefore 
applying the Blasius solution once more, the optimal installation point was found to be between 6 and 9 inches aft of the leading edge $(0.19<x \mid c<0.27)$.

With the pressure distribution and skin friction on the plate quantified and the transition point on the plate located, the third set of measurements, the laminar boundary layer profiles on a swept wing, were taken. To acquire these, the BLDS unit was affixed to the plate in-line with the free-stream direction of the tunnel test-section at a distance of 8 inches from the leading edge $(x \mid c=0.25)$. Another series of tests at maximum wind tunnel dynamic pressure was performed with the BLDS unit in total, rotatable, and Conrad configurations.

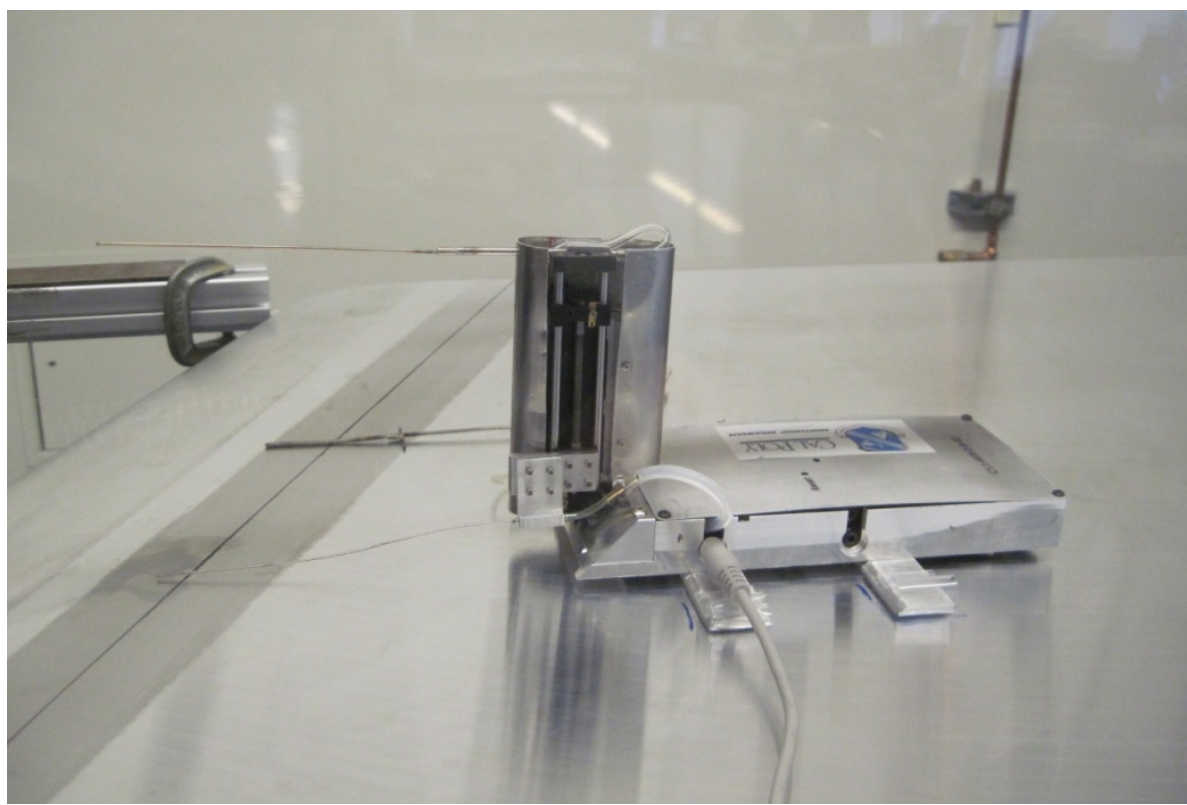

Figure 4.16 - Swept flat plate in 2'x2' wind tunnel with BLDS installed

Ten profiles were taken for each of the different anemometers and another direct comparison of the velocity magnitude measurements, similar to the one obtained from the unswept flat plate, was completed with the ten profile measurements for each probe shown in Figure 4.17 below. 


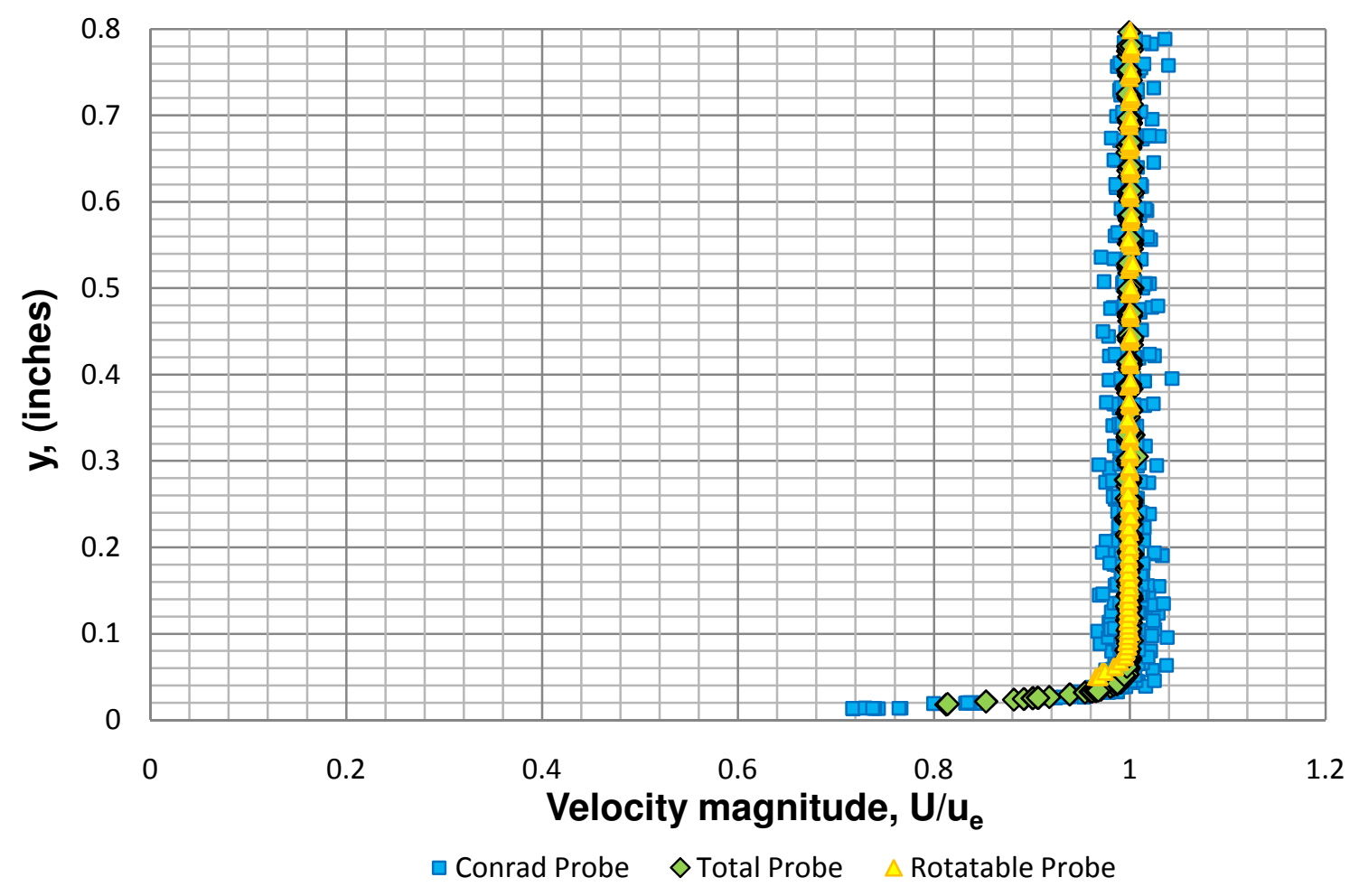

Figure 4.17 - Boundary layer profiles of velocity magnitude on elliptical nose swept flat plate at $\mathbf{x} / \mathrm{c}$ of 0.25 for total, rotatable, and Conrad probes

The velocity magnitudes measured by the Conrad, rotatable and total probes show agreement with respect to the shape of the boundary layer, which was laminar as expected. The Conrad probe results were found to be well within the uncertainty limit of \pm 0.02 . The estimated boundary layer height at this chord distance was found by applying the Blasius solution to a laminar boundary layer over a flat plate. The estimated height is 0.045 ", which agrees very well to the measured height of $\sim 0.05 "$.

Following the velocity magnitude comparison, an analysis of the $w \mid u_{e}$ component of the flow over the swept flat plate was done, the results of which are shown in Figure 4.18 below. In this case the probe was not yawed, but 
nominally aligned with the direction of flow in the wind tunnel. Due to the sweep of the plate and the results of the Sears calculation, the $w \mid u_{e}$ component was expected increase as the distance from the surface increased before becoming constant in the free-stream.

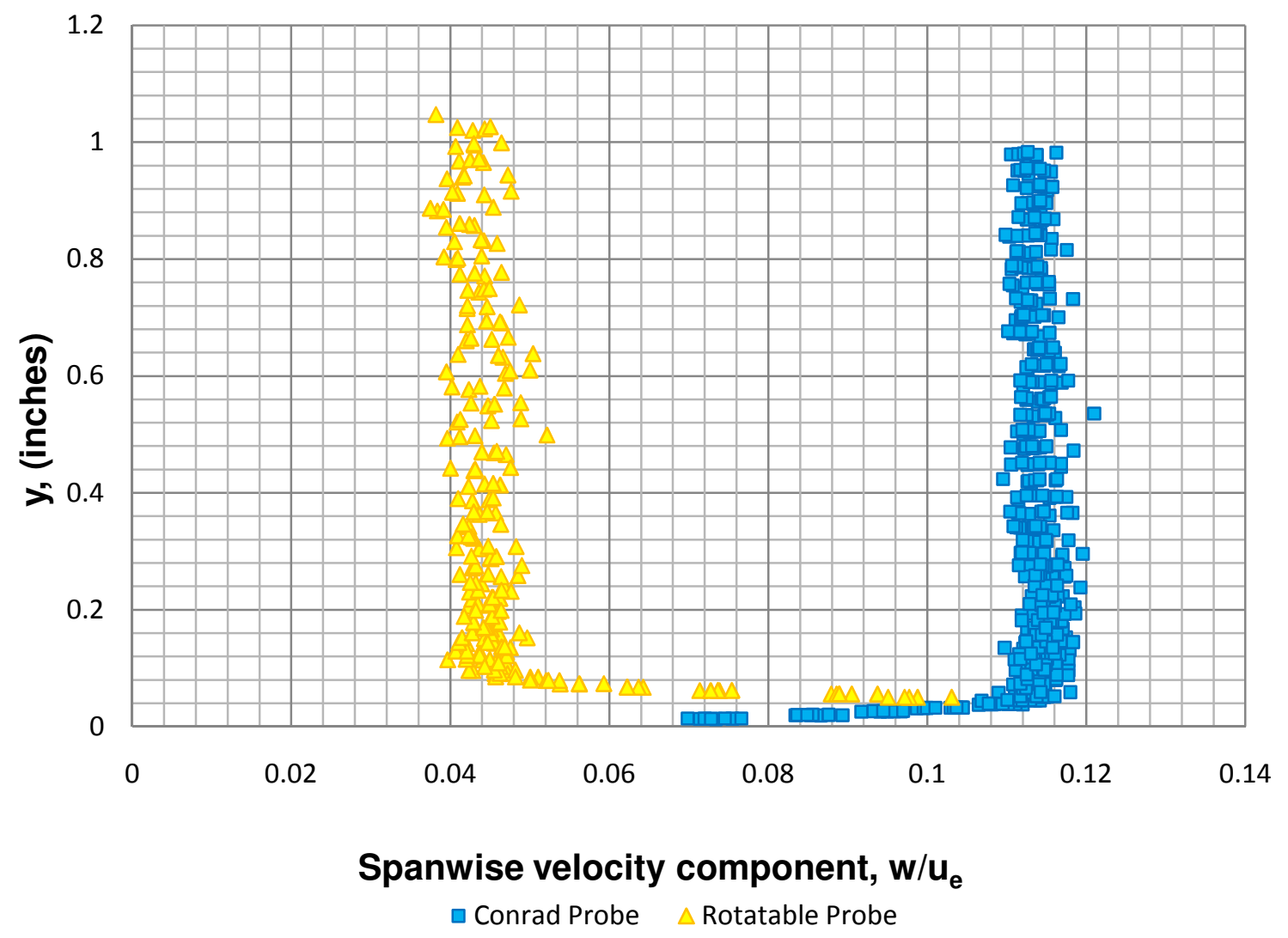

Figure $4.18-w \mid u_{e}$ component velocity profiles for rotatable and Conrad probes in 2'x2' wind tunnel @ $\mathrm{x} / \mathrm{c}=\mathbf{0 . 2 5}$ on elliptical nose swept flat plate

The Conrad probe successfully measured a change in the $w \mid u_{e}$ component of the velocity, perpendicular to the probe axis. The rotatable probe showed the same skewing effect as with the unswept flat plate results, with the cause being attributed to surface effects of the larger diameter probe being within two diameters of the surface and inside of a boundary layer only one diameter thick. 
The next comparison was made with the two measured yaw angles from the rotatable and Conrad probes. The yaw angle was predicted, by swept wing theory and the results of the Sears calculation, to change through the boundary layer and become constant in the free stream.

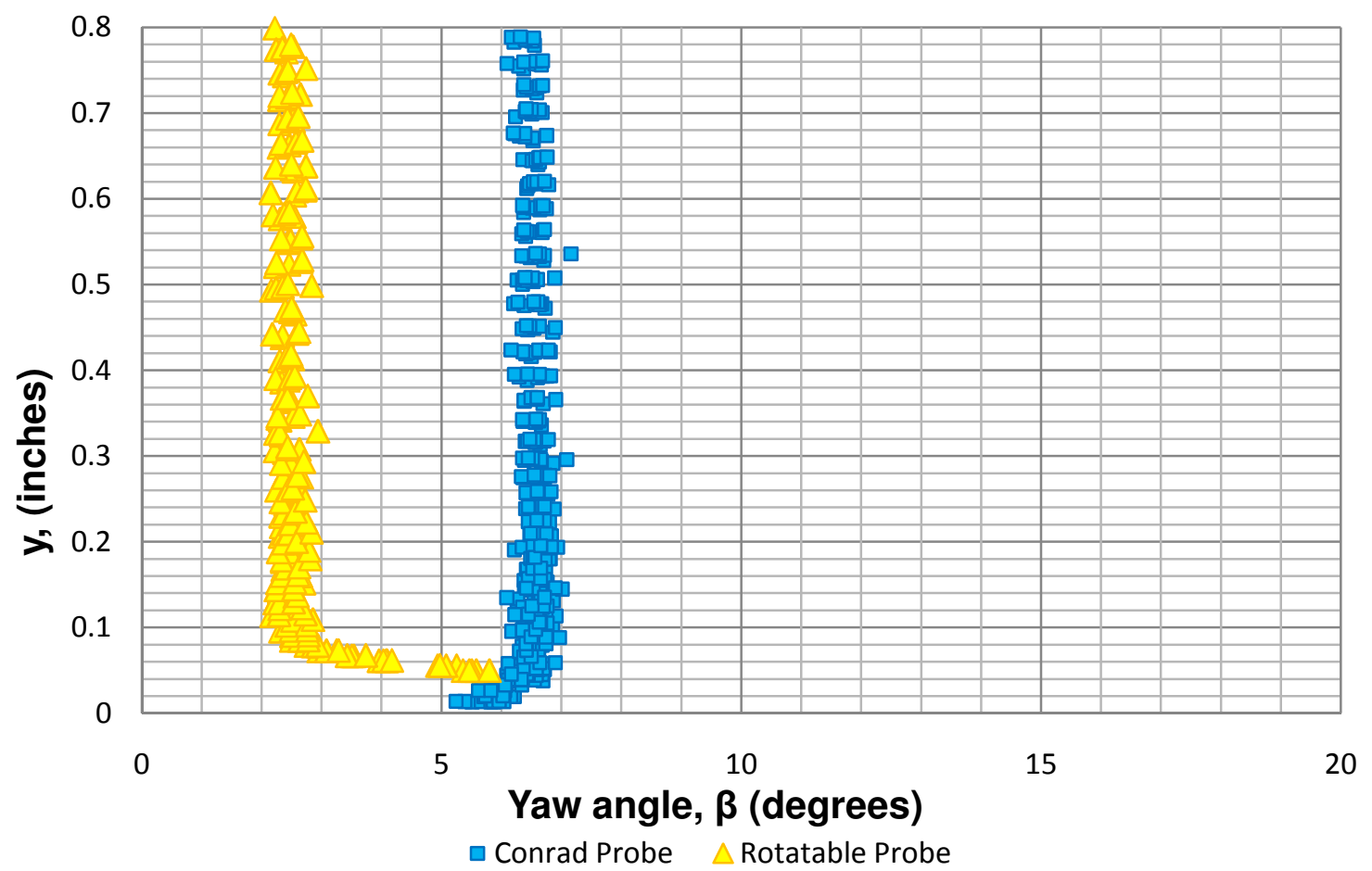

Figure 4.19 - Yaw angle, $\beta$ vs. height for Conrad probe in 2'x2' wind tunnel @ $\mathrm{x} / \mathrm{c}=\mathbf{0 . 2 5}$ on elliptical nose swept flat plate

The rotatable probe data once again shows signs of surface effects when within one probe diameter of the surface. As such, no change in the yaw angle was able to be reliably measured by this probe. The Conrad probe data confirms a change in flow angle with height, indicating that an appreciable amount of secondary flow was occurring on the swept plate. The measured Conrad probe yaw angle data fell within the range of the $\pm 1.25^{\circ}$ uncertainty bound, but exhibited a discrepancy of four degrees when compared to the rotatable data. 
The presence of this disparity in both the unswept and swept flat plate results corroborates the presence of irregularities in the manufacture of the Conrad probe.

The Conrad probe data from both the unswept and swept flat plate results did exhibit scatter in the case of both the velocity magnitude and the yaw angle. This scatter can be explained by the range of the pressure sensors attached to the BLDS and the average pressure measured by the Conrad probe. The average pressure in the two tubes of the Conrad probe was found in Figure 2.11 to be approximately 0.58 , or $58 \%$ of free-stream; this means that only about half of the flow approaching the probe is measured by the pressure sensor, decreasing the range of the sensor on the BLDS from $15 \%$ to $8 \%$. In the uncertainty analysis Chapter 3, a lower usage of the measureable range of the sensor amounted to a higher degree of uncertainty in the measurement, which would account for the additional scatter in the Conrad probe data. This conclusion is supported by the data obtained from the rotatable and total probes; the total probe, with an average pressure of 1.0 and rotatable probe, with an average pressure of 0.8 [4], both show much less scatter than the Conrad, therefore it is reasonable to assume that the increased sensing range of these probes due to their increased average pressure decreases their uncertainty. With the Conrad probe successfully able to measure velocity magnitude and also resolve in-plane flow components on both swept and unswept scenarios, the next progression was to utilize the probe for its intended application as a faster and more efficient way to measure boundary layers in a flight test scenario. 


\section{FLIGHT TEST RESULTS}

In cooperation with Northrop-Grumman Corporation, Scaled Composites, and sponsored by the United States Air Force, the Cal Poly BLDS research group, consisting of Dr. Russell Westphal, Drew Hutcheson, Jonathan White, and Rocky Ulk, was invited to take measurements as part of a flight test examining flow conditions and validating the existence of laminar flow on a swept model [34][35][36]. The BLDS group provided support to the project in the form of boundary layer and skin friction data to supplement data obtained using infrared imaging on a swept model. The swept wing model was flown to altitude using the payload section beneath the fuselage of Scaled Composites' White Knight I aircraft.

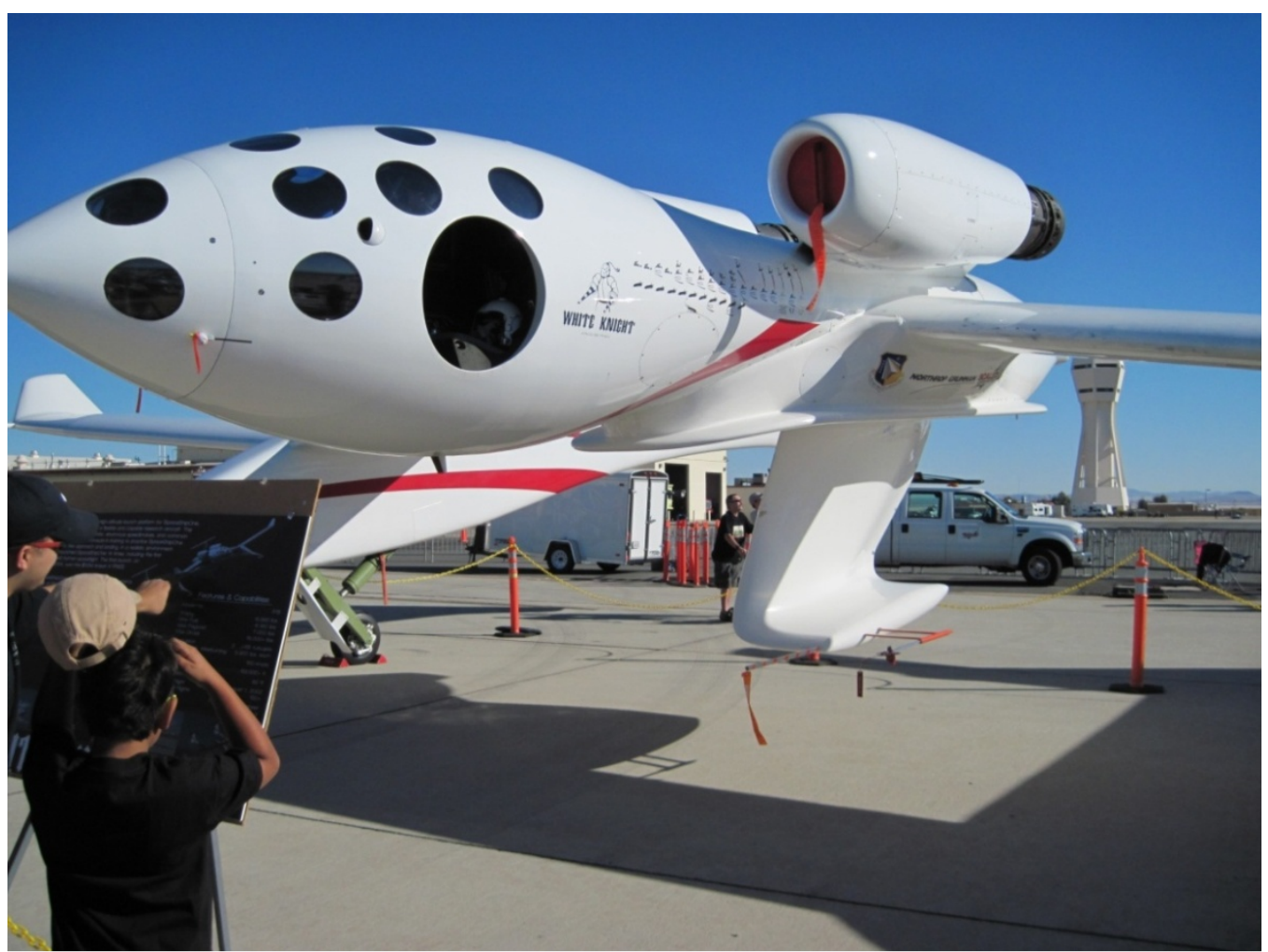

Figure 5.1 - Scaled Composites' White Knight I aircraft with attached swept wing test section 
The swept wing model had a $30^{\circ}$ forward sweep and a 90 inch chord with predicted Reynolds numbers of 1.07e5, 7.92e4, and 7.19e4 (per inch), at altitudes of $34,000,41,000$, and 43,000 feet [34]. Figure 5.2 shows a diagram of the swept wing model and Table 5.1 shows a summary of the flight tests.

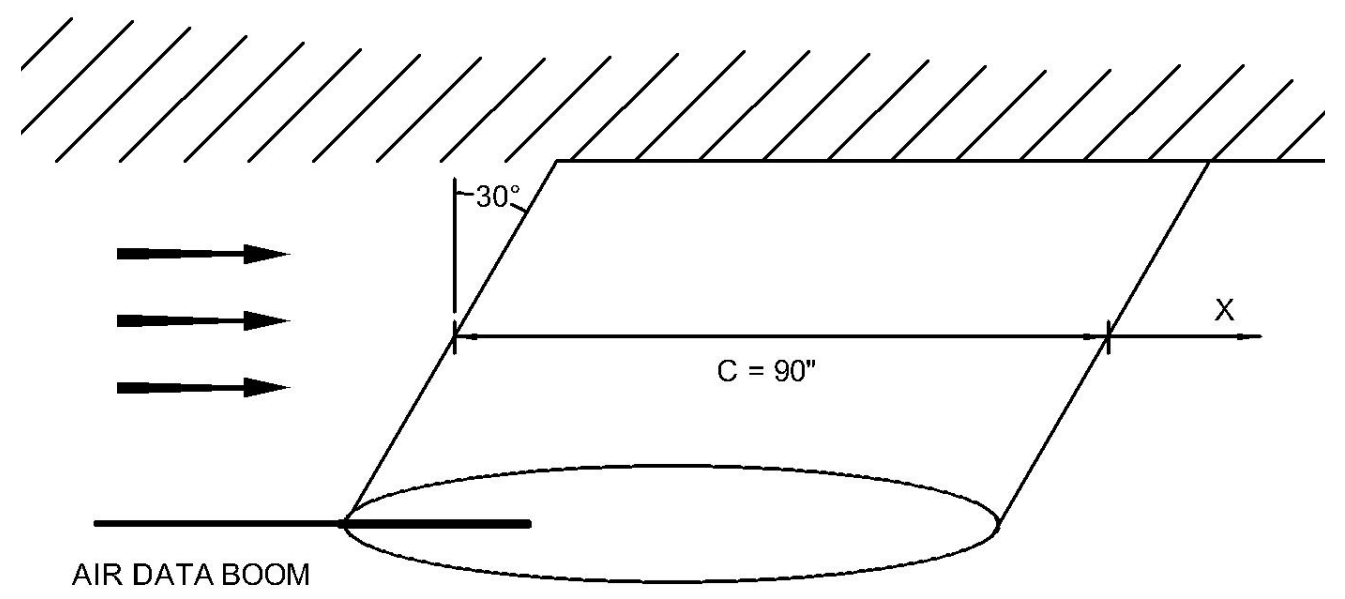

Figure 5.2 - Diagram of swept wing model

Table 5.1 - Test Matrix for Flight Test

\begin{tabular}{|c|c|c|c|c|}
\hline Altitude & $\begin{array}{c}\text { Probes } \\
\text { Used }\end{array}$ & Location on Model & $\begin{array}{c}\text { Reynolds } \\
\text { Number }\end{array}$ & $\begin{array}{c}\text { Mach } \\
\text { Number }\end{array}$ \\
\hline 34,000 feet & $\begin{array}{l}\text { Conrad \& } \\
\text { Rotatable }\end{array}$ & $\begin{array}{c}36.9 \text { inches aft of leading } \\
\text { edge }\end{array}$ & $3.95 \mathrm{e} 6$ & 0.52 \\
\hline 41,000 feet & $\begin{array}{c}\text { Conrad, } \\
\text { Total, \& } \\
\text { Rotatable }\end{array}$ & $\begin{array}{c}36.9 \text { inches aft of leading } \\
\text { edge }\end{array}$ & $2.92 \mathrm{e} 6$ & 0.52 \\
\hline 43,000 feet & $\begin{array}{c}\text { Conrad \& } \\
\text { Rotatable }\end{array}$ & $\begin{array}{r}36.9 \text { inches aft of leading } \\
\text { edge }\end{array}$ & $2.65 \mathrm{e} 6$ & 0.52 \\
\hline
\end{tabular}


The flight test parameters called for BLDS data to be taken at the three altitudes with sideslip angles (aircraft yaw angles) of 5.8, 6.4, and 7.0 degrees. Due to the vertical mounting position of the model, the varying sideslip angles were equivalent to varying angles of attack, which had to be changed to reach target pressure distributions on the model. For these various flight tests the BLDS unit was placed on the upper surface of the model at a nominal chord distance of $41 \%$. This location was measured by the BLDS in its total, rotatable and Conrad probe configurations.

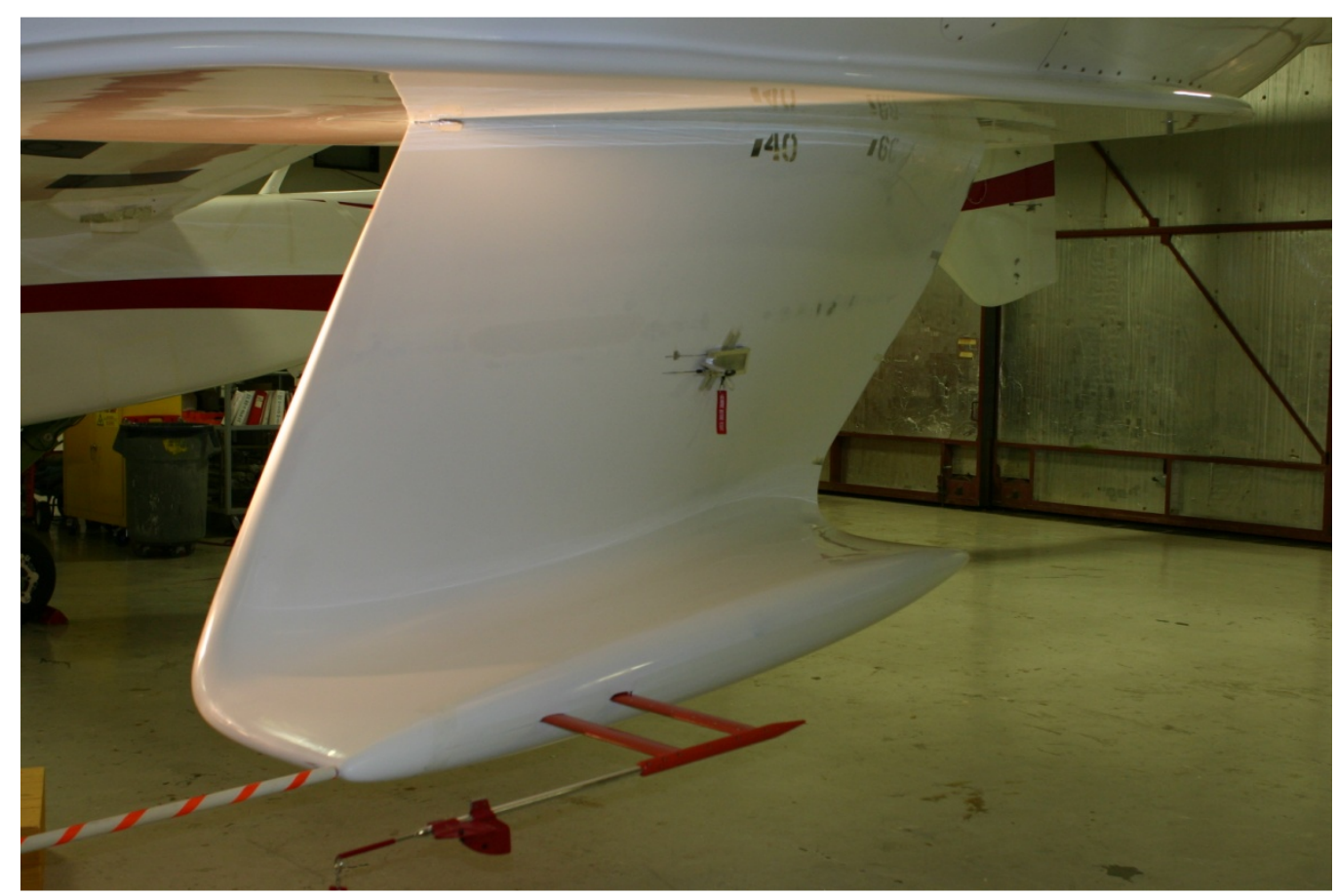

Figure 5.3 - BLDS unit attached to swept wing test section on Scaled Composites' White Knight I aircraft 


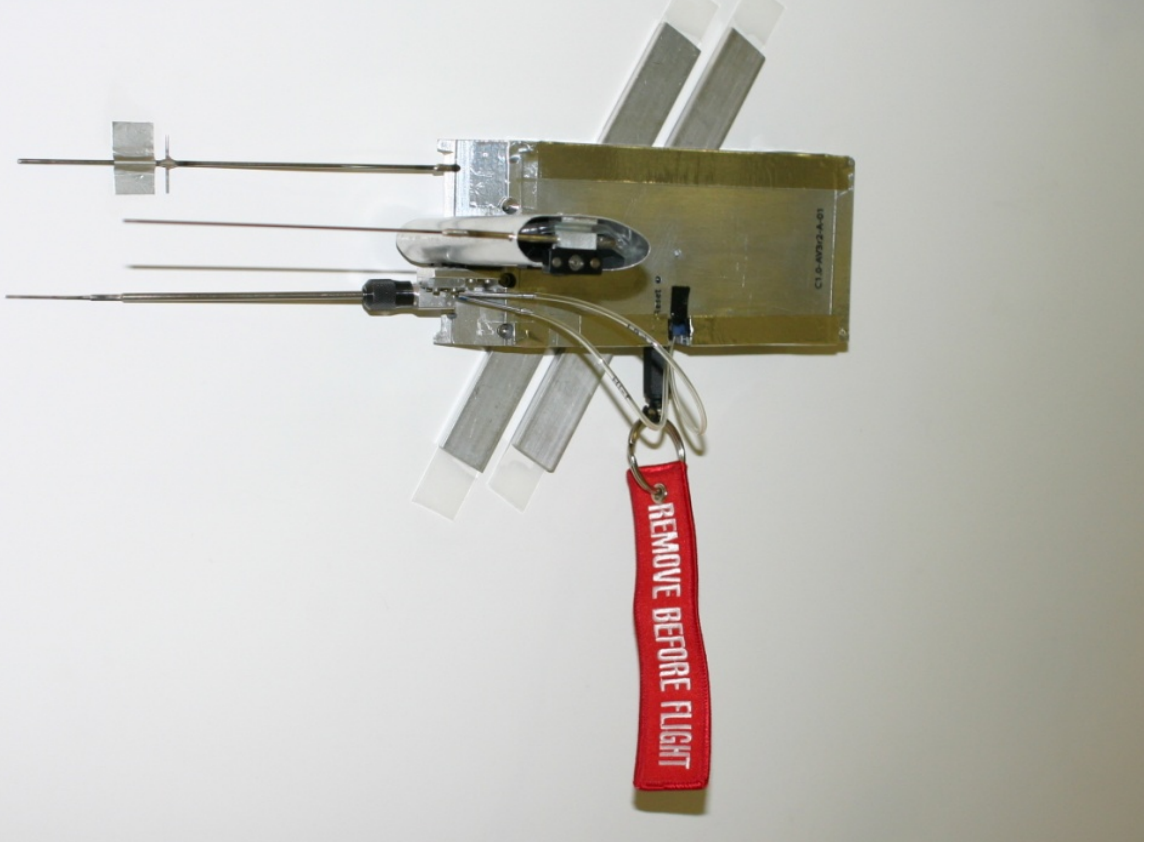

Figure 5.4 - Close-up view of BLDS unit in Conrad probe configuration attached to swept wing test section on Scaled Composites' White Knight I aircraft

From these three data sets a large amount of data was successfully recovered. After collecting and parsing all of the collective data sheets, the results were all combined to provide both an examination of flow conditions on the test article with three different measurement schemes and to evaluate the different probe types. The first set of results was a direct comparison of the velocity magnitudes obtained by all three probes at a sideslip angle of $6.4^{\circ}$. As seen in Figure 5.5, all three probes show a mix of laminar and turbulent boundary layers for the duration of flight at altitude conditions, where climb and descent are neglected. Examining the laminar boundary layers, the results appear to be in good agreement with one another, showing that the Conrad probe was able to measure a laminar boundary layer 0.06 " thick on the model. 


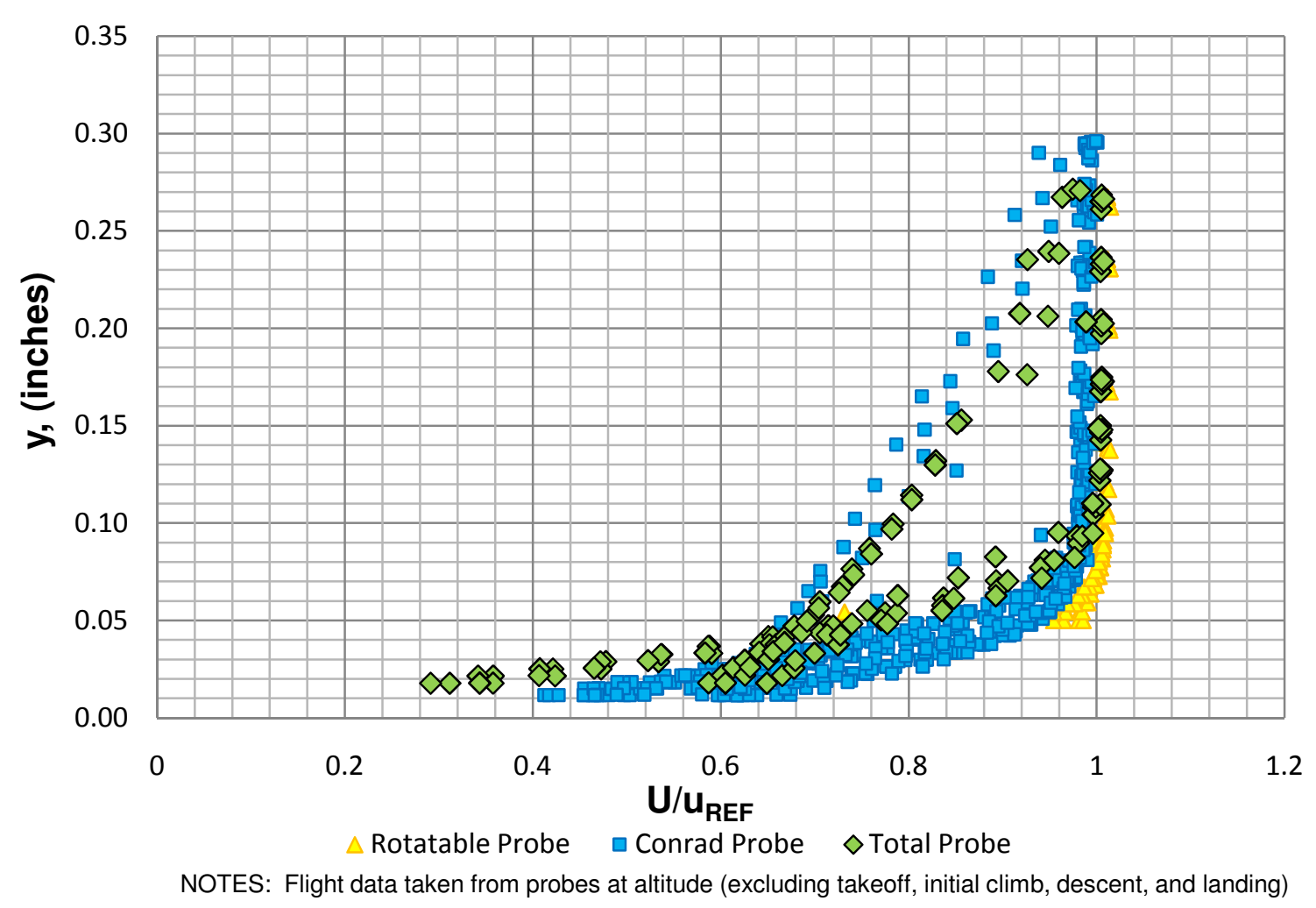

Figure 5.5 - Corrected velocity profiles for total, rotatable, and Conrad probes at altitude for flights \#14, \#15, \& \#16

The next set of data taken at a sideslip angle of $6.4^{\circ}$ shows both of the inplane velocity components on the swept test article. These components indicate how the boundary layer behaves at this particular region on the test section and also give insight into the amount of secondary flow achieved. Where it was practical, a comparison of all three data sets (total, rotatable, and Conrad) was made; however in the case of measuring the $w \mid u_{\text {REF }}$ or component of velocity measured parallel to the leading edge, only the rotatable and Conrad probe results are shown. In cases where the $w \mid u_{\text {REF }}$ component of the velocity was measured, the data was corrected by way of a coordinate transformation to change the measurements from probe axis coordinates to free-stream axis coordinates in order to get a more direct comparison of the profiles. From these 
results, the low altitude measurements shown in Figure 5.6 demonstrate that both the Conrad and rotatable probes were able to measure a laminar boundary layer in the direction normal to the leading edge of the test section. A similar result is shown for the span-wise velocity component in Figure 5.6 (b), which in both data sets shows the velocity changing with respect to distance from the surface, indicating the presence of secondary flow. Both measurements show a slim laminar boundary layer developing with a thickness of roughly 0.05 inches on the wing. The next sets of measurements were taken at the middle altitude condition at 41,000 feet; these measurements were also carried out at a sideslip angle of $6.4^{\circ}$. In the case of the $\mathrm{u} \mid \mathrm{u}_{\mathrm{REF}}$ component of the boundary layer, total data was also taken, giving good comparison different probes. Figure 5.7 (a) illustrates the agreement between the probe measurements with a tight laminar grouping about the desired $u \mid u_{\text {REF }}$ value of 1 , or 100 percent of free-stream velocity and an increased thickness of $\sim 0.06 "$. Once again, secondary flow is shown to be occurring with the amount of flow turning being fairly consistent between the two initial data sets. The next sets of data were obtained at the highest altitude case, nominally 43,000 feet. The results, shown in Figure 5.8, are similar to those of the lower altitudes, with laminar boundary layers measured in both the $x, u$ and $z, w$ directions. The laminar boundary layer again increases slightly in thickness to $\sim 0.07$ " at the higher altitude. The w|u $\mid u_{\text {REF }}$ component again shows the presence of flow turning occurring within the boundary layer on the swept model. 
All of the data sets for the Conrad probe appear to be within the uncertainty limits of \pm 0.01 established for flight tests in Chapter 2 . Additionally it is noted that the scatter exhibited by the wind tunnel data in Chapter 4 is not present in the flight test data, validating the assumption of pressure sensor scale affecting uncertainty. The data is useful in demonstrating the robustness of the three probe designs in that they can successfully measure the in-plane components of boundary layers in different flight conditions. 

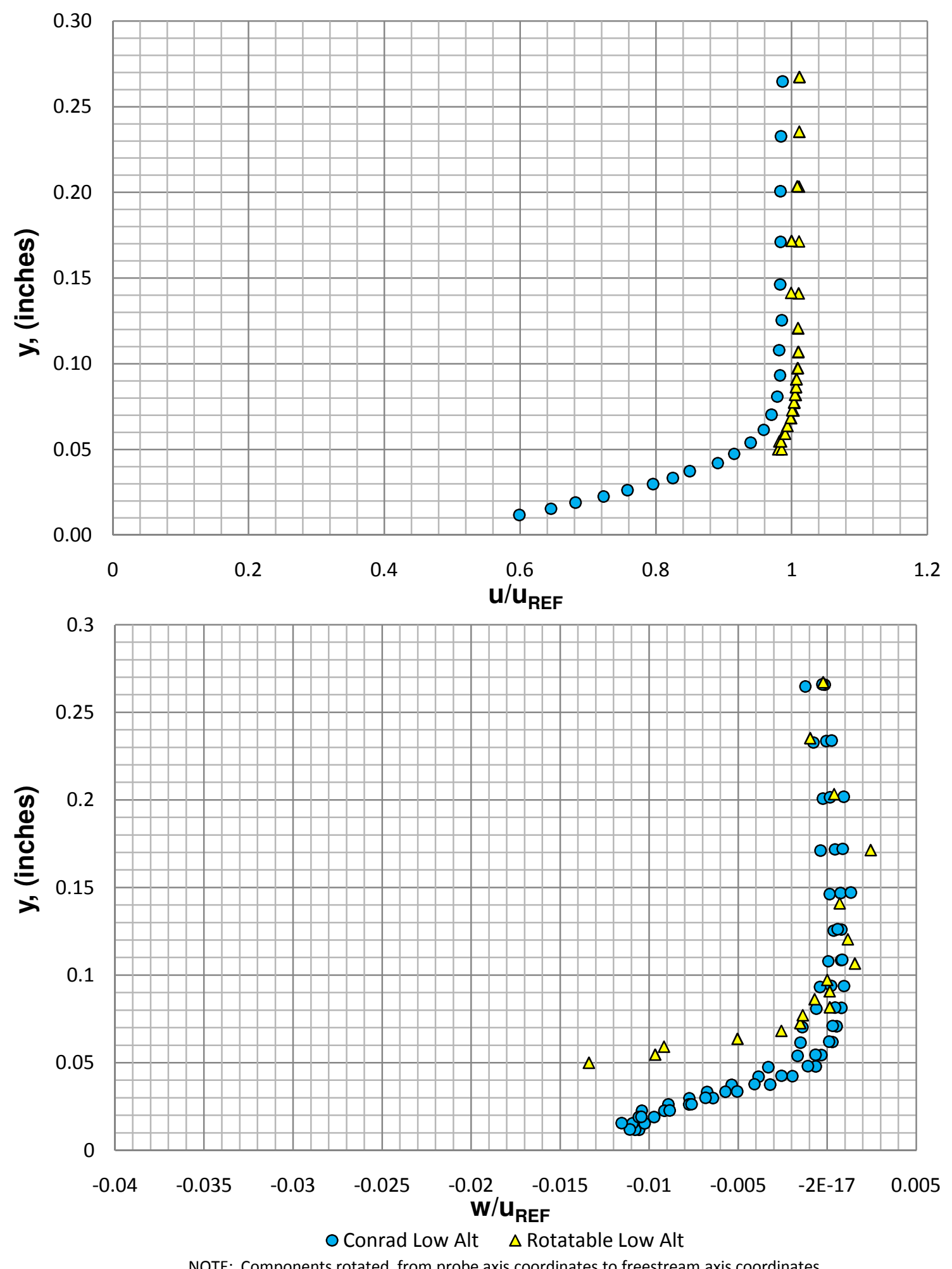

Figure $5.6-$ (a) $u / u_{R E F}$ vs. $y$ and (b) $w / u_{R E F} v s . ~ y$ at sideslip angle of $6.4^{\circ}$ for Conrad and rotatable probes at low altitude condition ( $x / c=0.41$, text article upper surf.) for flights \#14 \& \#15 


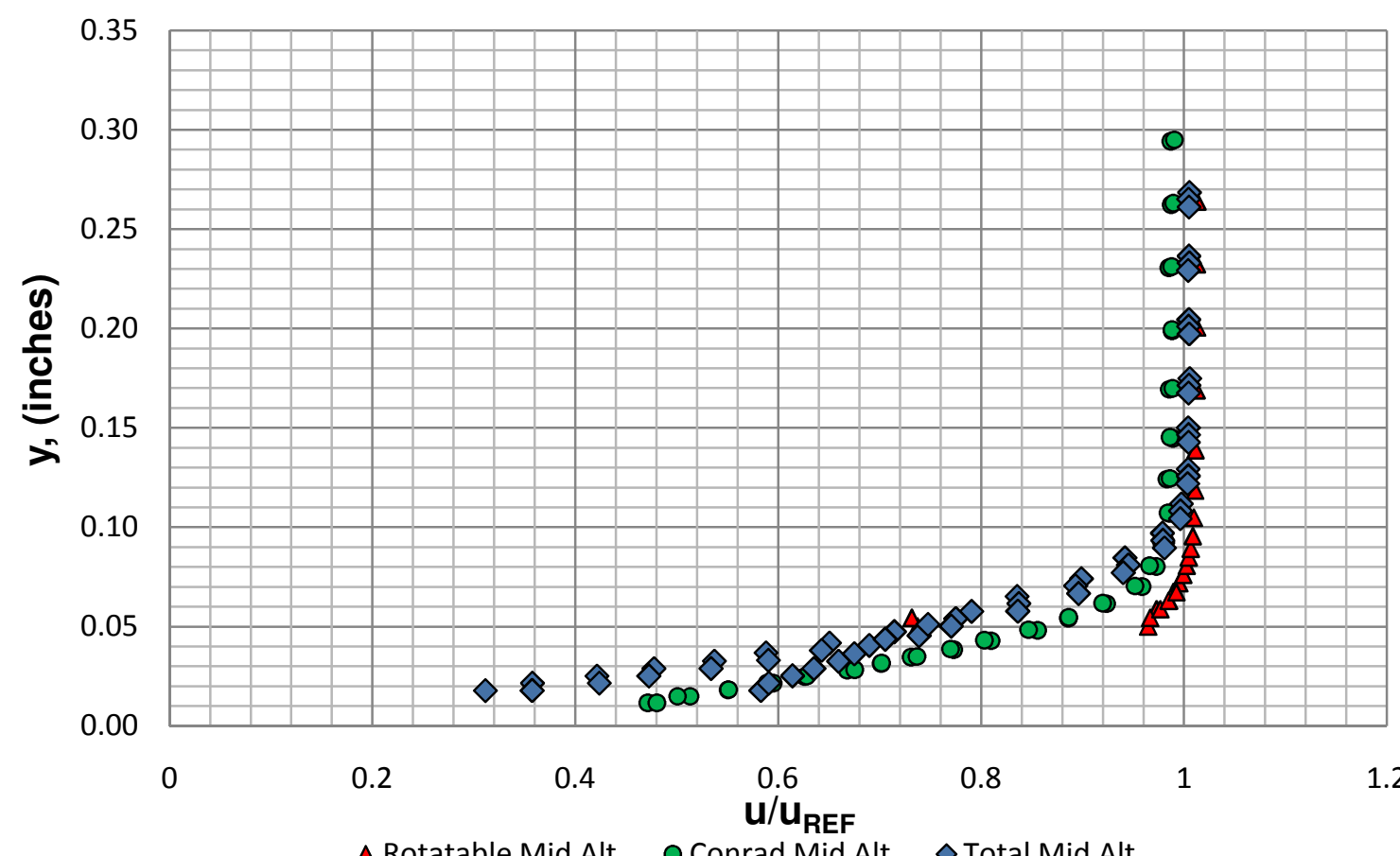

1.2

$\Delta$ Rotatable Mid Alt $\quad$ O Conrad Mid Alt $\diamond$ Total Mid Alt

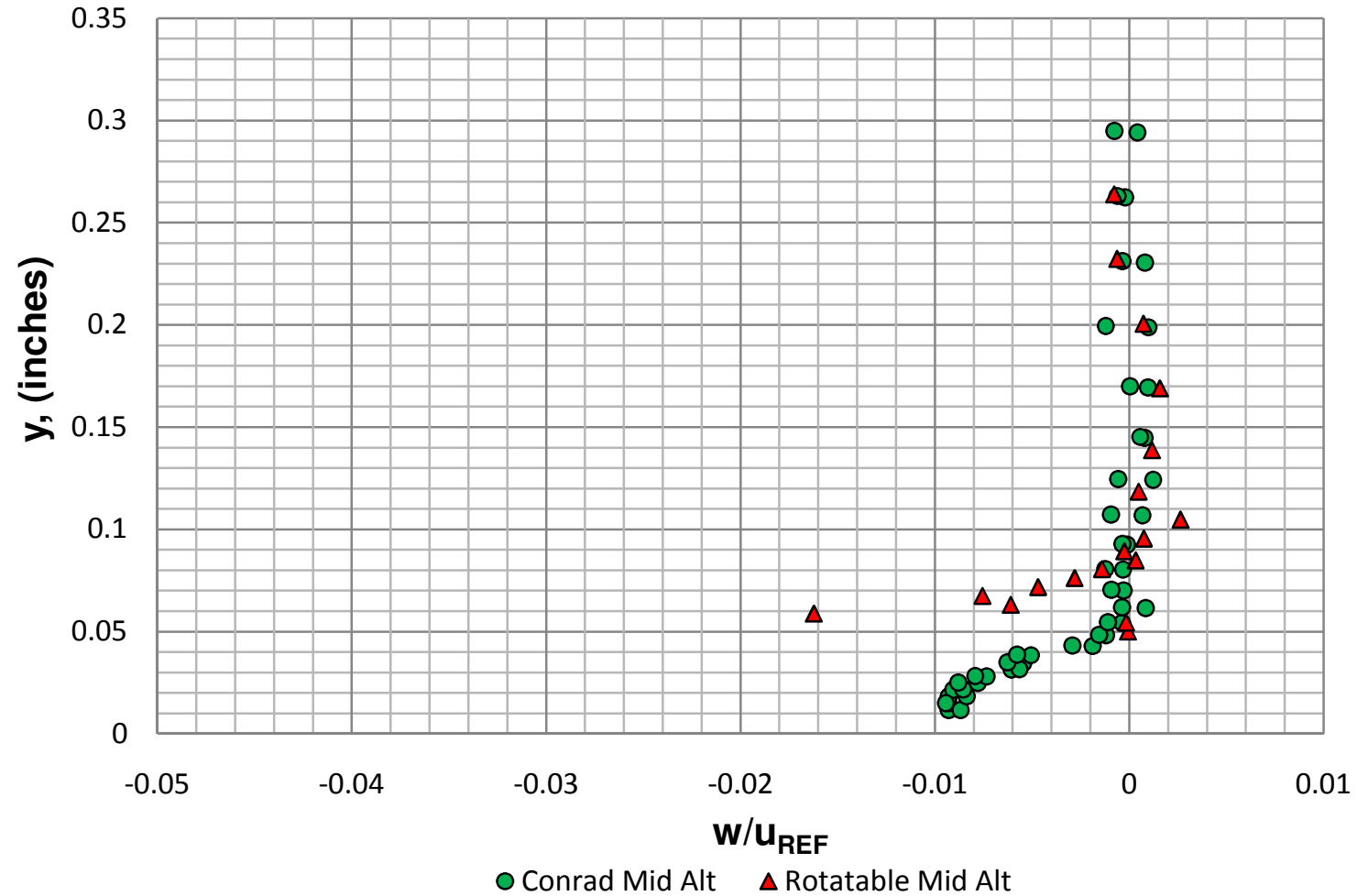

NOTE: Components rotated from probe axis coordinates to freestream axis coordinates

Figure 5.7 - (a) u| $\mathrm{u}_{\mathrm{REF}}$ vs. y for total, Conrad, and rotatable and (b) $\mathrm{w} \mid \mathrm{u}_{\mathrm{REF}}$ vs. $y$ for Conrad and rotatable probes at sideslip angle of $6.4^{\circ}$ at middle altitude condition ( $x / c=0.41$, text article upper surf.) for flights \#14,\#15, \& \#16 

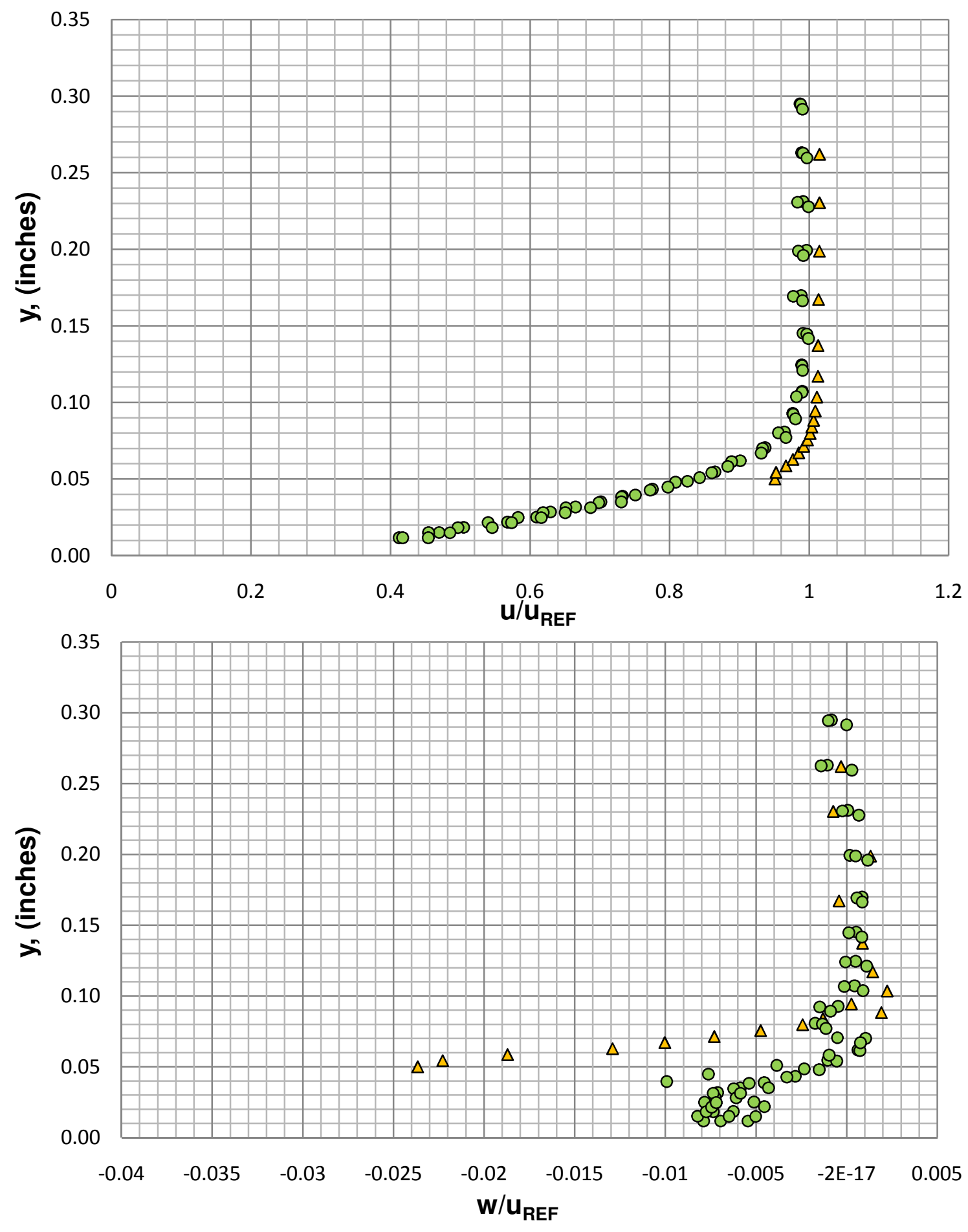

$\Delta$ Rotatable High Alt o Conrad High Alt

NOTE: Components rotated from probe axis coordinates to freestream axis coordinates

Figure $5.8-(a) \mathrm{u} / \mathrm{u}_{\mathrm{REF}} \mathrm{vs.} \mathrm{y}$ and (b) $\mathrm{w} / \mathrm{u}_{\mathrm{REF}} \mathrm{vs.} \mathrm{y}$ at sideslip angle of $6.4^{\circ}$ for Conrad and rotatable probes at high altitude condition ( $x / c=0.41$, text article upper surf.) for flights \#14 \& \#15 
All of the acquired data indicates a few key points with respect to both the test section and the efficacy of the Conrad probe for use in boundary layer measurements. The test section boundary layer is definitely laminar at $41 \%$ chord; this laminar condition is found at all three test altitudes, at different Reynolds numbers. Additionally, the measured boundary layers in the directions normal and parallel direction to the leading edge indicate that the test section has secondary flow as predicted by swept wing theory.

The Conrad probe was able to successfully measure laminar boundary layers of $\sim 2 \mathrm{~mm}$ thickness in all of the test conditions. The boundary layer profiles obtained all compare well with profiles obtained with the total configuration of the BLDS device, indicating that the Conrad probe can measure flow velocity magnitude with an accuracy that is adequate for defining boundary layer thickness and state. The Conrad probe was also able to resolve the inplane velocity components over the model surface. The results from the rotatable probe support this to some degree, however it appears the larger 1.3 $\mathrm{mm}$ diameter of this probe limited flow angle measurements inside of the boundary layer, thus truncating results from this probe. The smaller 0.02 " diameter of the Conrad probe appears to have aided the probe in measuring inside of the thin boundary layer, with measurements taken to well within two probe diameters of the surface. Comparable results to the Conrad probe would likely be obtained by the rotatable probe given a smaller diameter tip, however faster data acquisition time and smaller power consumption favor the Conrad probe in this case. 


\section{CONCLUSIONS}

A pressure probe anemometer design pioneered by O. Conrad [9] and refined by Brebner [10] was used to construct a 0.064" diameter prototype Conrad probe. This probe was used on the boundary layer data system to measure in-plane flow velocity magnitude and direction. In order to properly measure the flow yaw angle and velocity magnitude a novel non-nulling calibration was developed, calibrating the probe in a flow field with a dynamic pressure of $30 \mathrm{psf}$ over a range of \pm 30 degrees. The calibration technique made use of non-dimensional coefficients derived from measured tube pressures, which were subjected to polynomial fits over the measured angular range. In order to accommodate the Conrad probe on the BLDS, changes to the hardware and software configurations were made. An additional pressure sensor with a range of \pm 1.5 psid was added to the BLDS device to measure the pressure difference between the left and right tubes of the probe. To attach the probe to the stage traverse on the device, a collet-head bracket that allowed for quick removal of probes was used. Modification existing BLDS software was done to incorporate the new sensor input and a new sub-routine for Conrad probe data collection was created.

To shakedown the newly configured device and validate the calibration method a series of tests were performed in a 2'x2' square wind tunnel at an operational maximum dynamic pressure of 30 psf. The unit with the 0.064 " prototype probe attached was tested in a yawed configuration on the bottom 
surface of the tunnel. A turbulent boundary layer $\sim 0.5$ " thick was measured by both the Conrad probe and a total pressure probe, validating the velocity magnitude measurement. A constant yaw angle was also recorded, indicating that the prototype probe and calibration were working properly. Another set of wind tunnel tests were conducted to measure laminar boundary layers on a 0.75 " thick flat plate with an elliptical leading edge and 48 inch chord. To increase spatial resolution within the thin laminar boundary layer, a smaller 0.020 " diameter probe was constructed and calibrated. Laminar testing with the smaller probe was performed on the plate at a location 12 inches aft of the leading edge. The measured shape of the boundary layer was laminar and showed a thickness of 0.08 ", which agreed well with the estimated thickness (from Blasius) of 0.07 ". In addition to velocity magnitude results, the probe was also able to measure a constant yaw angle and the in-plane velocity components in the thin laminar boundary layers. To simulate measurement of boundary layers and secondary flow on a swept wing, a 0.5 " thick, $30^{\circ}$ swept flat plate with an elliptical leading edge and a chord of 33 inches was designed and constructed. The 0.020 " diameter probe was used again to measure boundary layers at a location 8 inches aft of the leading edge. A laminar boundary layer 0.045 " thick was predicted which agreed well with the measured thickness of 0.05 ". The yaw angle of flow on the plate was found to vary as the probe traversed the boundary layer, confirming the presence of secondary flow on the plate and showing agreement with traditional three-dimensional boundary layer theory. 
With the calibration tested and validated in both swept and unswept conditions, the BLDS and Conrad probe were used in a series of flight tests on a $30^{\circ}$ swept wing test model attached to Scaled Composites' White Knight I aircraft. The 90 inch chord model was flown to altitudes of $34 \mathrm{k}, 41 \mathrm{k}$, and $43 \mathrm{k}$ feet at angles of attack of 5.8,6.4, and 7.0 degrees. Results from these tests assisted in confirming the extent of laminar flow on the test section at mid-span at a distance of 37 inches aft of the leading edge. Laminar boundary layers nominally $0.05 ", 0.06$ ", and 0.07 " thick were measured at the three test altitudes. In-plane velocity components were also measured that showed the expected change in the span-wise, $w \mid u_{\mathrm{REF}}$ through the boundary layer indicative of secondary flow.

Successful measurement of both flow velocity magnitude and angle coupled with the fast data acquisition time and low energy consumption demonstrate the usefulness of the Conrad probe in the measurement of boundary layers and the furthering of laminar flow research. 


\section{REFERENCES}

[1] Westphal, R.V., Bleazard, M., Drake, A., Bender, A.M., Frame, and Jordan, S. R., "A Compact, Self-Contained System for Boundary Layer Measurement inFlight," AIAA-2006-3828, AIAA Meeting Papers on Disc [CD-ROM], Vol. 11, No. 10-13, AIAA, Reston, VA, 2006.

[2] Bender, A. M., Drake, A., Westphal, R.V., Jordan, S.R., "Development and Flight Demonstration of Self-Contained Boundary Layer Measurement Devices," AIAA-2008-7333, AIAA Meeting Papers on Disc [CD-ROM], Vol. 13, AIAA, Reston, VA, 2008.

[3] Bender, A. M., Westphal, R.V., and Drake, A., "Application of the Boundary Layer Data System on a Laminar Flow Swept Wing Model In-Flight," in preparation, 2010.

[4] White, F. M., "Viscous Fluid Flow", Mcgraw-Hill, Third Edition, 2006

[5] Chu, J. K., and Young, A. D., "A Further Investigation of Viscous Effects in a Wing-Plate Junction," Queen Mary College (University of London), QMC ER1003, March 1975.

[6] Westphal, R. V. and Ortega, A., "Cascade Throat Flow Measurements Using a Rotatable Single-Hole Pressure Probe", AIAA-2004-2601, AIAA Conference Proceedings Series, available online, AIAA, Reston, VA. 2004.

[7] Westphal, R. V., Prather, M., and Toyooka, M., "Rotatable Single-Hole Pressure Probe for Flow Velocity and Direction," AIAA-2002-3128, AIAA Conference Proceeding Series, available online, AIAA, Reston, VA, 2002.

[8] Westphal, R.V., Frame, D., Jordan, S., Wanner Jr., A., Thompson, B., Bender, A. M., and Drake, A., "Design of a Third-Generation Boundary Layer Measurement System for In- Flight Measurements," AIAA-2008-7332, AIAA Meeting Papers on Disc [CD-ROM], Vol. 13, AIAA, Reston, VA, 2008.

[9] Conrad, O. "Gerate zur Messung von Stromungrichteungen", Archiv fur Technisches Messen, 116-200, October 1950

[10] Brebner, G.G. "Pressure and Boundary layer Measurements on a 59 Sweptback Wing at Low Speed and Comparison with High Speed Results on a $45^{\circ}$ Swept Wing", C.P. No. 86, Aeronautical Research Council, Her Majesty's Stationary Office, 1952. 
[11] Brebner, G.G., and Wyatt, L.A., "Boundary Layer Measurements at Low Speed on Two Wings of $45^{\circ}$ and $55^{\circ}$ Sweep", C.P. No. 554, Aeronautical Research Council, Her Majesty's Stationary Office, 1961.

[12] Black, J., "Pressure Distribution and Boundary Layer Investigations on 44 degree Swept-back Tapered Wing", C.P. No. 137, Aeronautical Research Council, Her Majesty's Stationary Office, 1953.

[13] Seddon, J. and Trebble, W.J.G., "Experiments on the Flow into a Swept Leading-edge Intake at Zero Forward Speed with Notes on the Wider Uses of a Slotted Intake", Reports and Memoranda No. 2909, Aeronautical Research Council, Her Majesty's Stationary Office, 1954

[14] Horlock, J.H., "Instrumentation Used in Measurement of the Three Dimensional Flow in an Axial Flow Compressor", C.P. No. 321, Aeronautical Research Council, Her Majesty's Stationary Office, 1957.

[15] Bryer, D.W., Walshe, D.E., and Garner, H.C., "Pressure Probes Selected for Three-Dimensional Flow Measurement", Reports and Memoranda No. 3037, Aeronautical Research Council, Her Majesty's Stationary Office, 1958.

[16] Bryer, D. W., and Pankhurst, R. C., 1971, "Pressure-Probe Methods for Determining Wind Speed and Flow Direction", Her Majesty's Stationary Office, London.

[17] Winkelmann, A.E., "Experimental Studies of a Two and a Threedimensional Low Speed Turbulent Boundary Layer", PhD Thesis, U of Maryland, May 1976.

[18] Winkelmann, A.E., "An Experimental Study of Separated Flow on a Finite Wing”, AIAA-1981-1882-336, AIAA Atmospheric Flight Mechanics Conference, August 1981.

[19] Davis, D.O. and Gessner, F.B., "Experimental and Numerical Investigation of Supersonic Flow through a Square Duct", AIAA-1985-1622-891, AIAA $18^{\text {th }}$ Fluid Dynamics and Plasmadynamics and Lasers Conference, July 1985.

[20] Davis, D.O., "Experimental and Numerical Investigation of Steady, Supersonic Turbulent Flow through a Square Duct", MS Thesis, Dept of Mech. Eng., University of Washington, 1985.

[21] Davis, D.O., and Gessner, F.B., "Further Experiments on Supersonic Turbulent Flow Development in a Square Duct", AIAA-1987-1247-480, AIAA $19^{\text {th }}$ Fluid Dynamics, Plasma Dynamics and Lasers Conference, June 1987. 
[22] Sforza, P.M., Smorto, M.J., and Grenier, M., "Traverse Curvature Effects in Turbulent Boundary Layers", AIAA-1987-1252-524, AIAA $19^{\text {th }}$ Fluid Dynamics, Plasma Dynamics and Lasers Conference, June 1987.

[23] Frey, M.O., and Gessner, F.B., "Experimental Investigation of Coannular Jet Flow with Swirl Along a Centerbody", AIAA-1990-1622-322, AIAA $21^{\text {st }}$ Fluid Dynamics, Plasma Dynamics and Lasers Conference, June 1990.

[24] Gooden, J.H.M., and Gleyzes, C., "Experimental Study of the Flow Around Two Scaled 3D Swept Wings", AIAA-1997-2020-985, AIAA, 1997.

[25] Hold, A.E., Calay, R.K., and O'Brien, M., "Flow Generated by the Interaction of an Inlet and a Cross-flow", Journal of Wind Engineering and Industrial Aerodynamics, Vol. 88, pgs 1-23, February 2000.

[26] Hin Ho, S.S., "Subsonic Intake Duct Flows", PhD Thesis, Department of Aeronautical and Mechanical Engineering, The University of Salford, Januaray 1990

[27] Christiansen, T. and Bradshaw,P., "The Effect of Turbulence on Pressure Probes”, Journal of Physics E: Scientific Instruments, Vol. 14, pgs 992-997, 1981.

[28] Prahlad, T.S., "Yaw characteristics of Preston tubes", AIAA Journal, vol. 10 no. 3 pgs 357-359, March 1972.

[29] Figliola, R. S. and Beasley D. E., "Theory and Design for Mechanical Measurements", Fourth Edition, Wiley \& Sons Inc, 2006

[30] Moffat, R. J., "Uncertainty analysis in the planning of an experiment", Journal of Fluids Engineering, 107: 173-178, 1983

[31] Sproston, J.L., and Goksel, O.T., "The Calibration of a Surface Static Tube", Aeronautical Journal, February 1972.

[32] Sears, W. R., "Boundary Layers in Three-Dimensional Flow", Cornell University, Ithica NY, July 1954

[33] Rosenhead, L., "Laminar Boundary Layers", Dover Publications Inc., NY 1963

[34] Drake, A. and Solomon, W.D. Jr., "Flight Testing of a 30-Degree Sweep Laminar Flow Wing for a High Altitude Long Endurance Aircraft", AIAA-20104571, $28^{\text {th }}$ AIAA Applied Aerodynamics Conference, 28 June-1 July 2010, Chicago, IL. 
[35] Bender, A.M., Westphal, R.V., and Drake, A., "Application of the Boundary Layer Data System on a Laminar Flow Swept Wing Model In-Flight", AIAA-2010$4360,27^{\text {th }}$ AIAA Aerodynamics Measurement Technology and Ground Test Conference, 28 June-1 July 2010, Chicago IL.

[36] Bender, A., Elliott, R., Shinagawa, Y., Korntheuer, A., Drake, A., Westphal, R., Gerashchenko, McKeon, B., and Yoshioka, S., "An Approach to Measuring Step Excrescence Effects in the Presence of a Pressure Gradient", AIAA-20100373, AIAA Meeting Papers on Disc [CD-ROM], Vol. 15, AIAA, Reston, VA, 2010. 


\title{
APPENDIX A: BLDS Program Code for Conrad Probe
}

\author{
//BLDS-A-NRC CPSU AV3 1-SEPTEMBER-2009 \\ //uses BLDS-A universal BLDS/PTDS board \\ //-AV3 board ONLY!! \\ //Combination non-rotating, rotatable single-hole probe, \& conrad probe ("NRC") version \\ //STARTUP

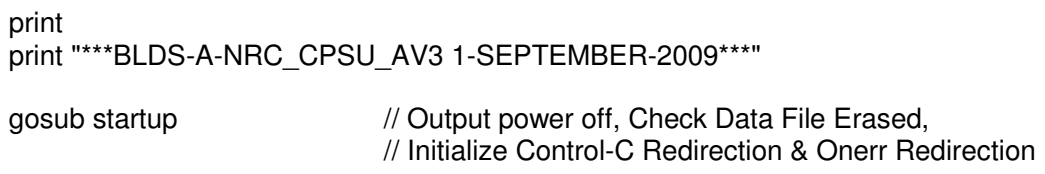




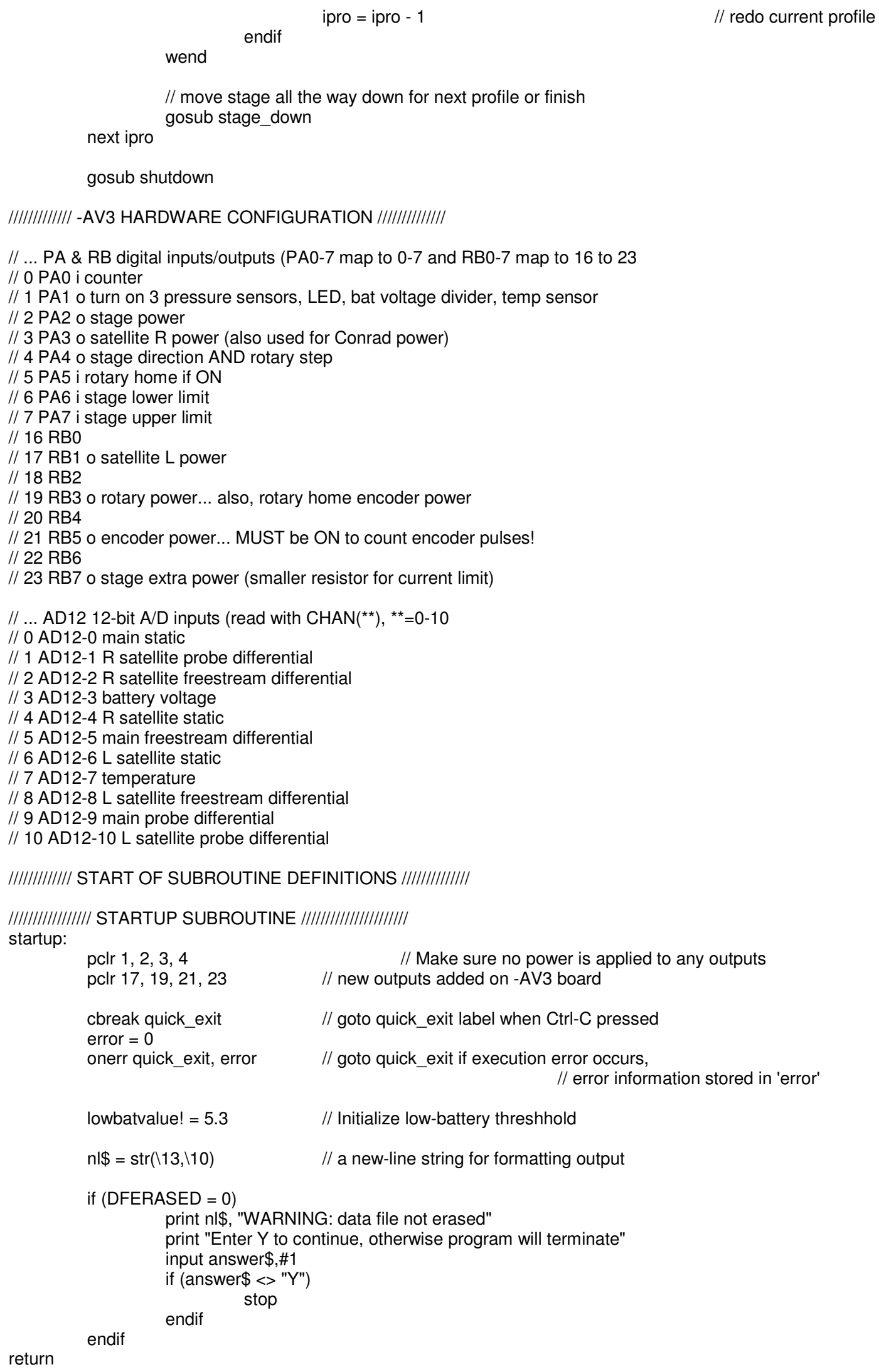

pclr 1, 2, 3, 4

pclr 17, 19, 21, 23

cbreak quick_exit 


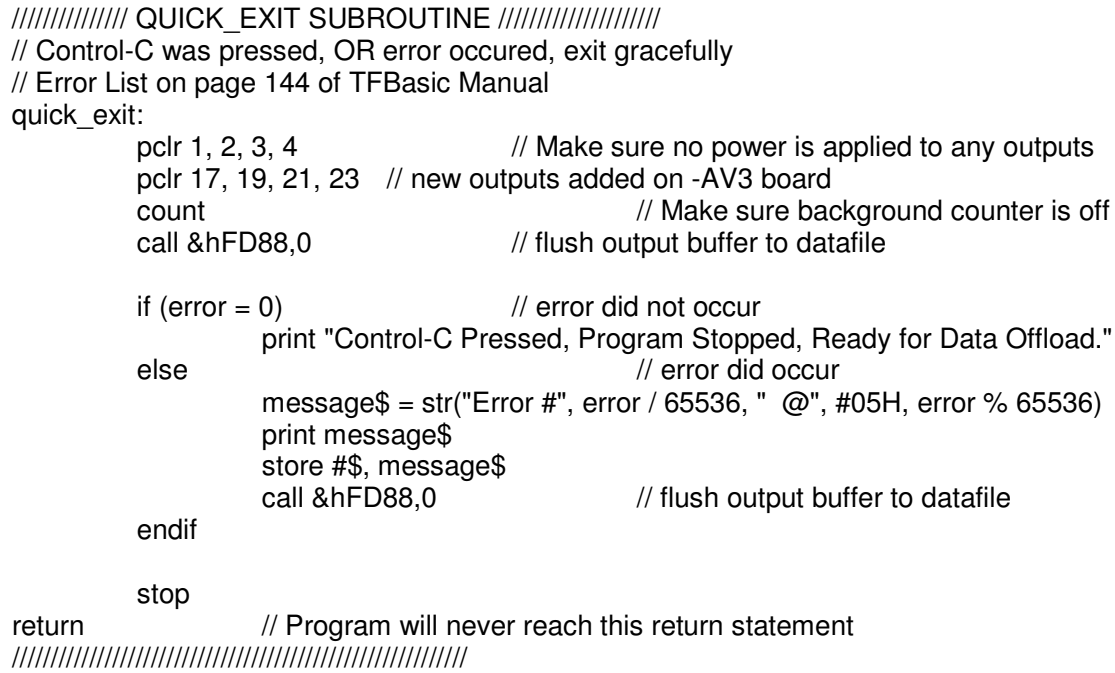




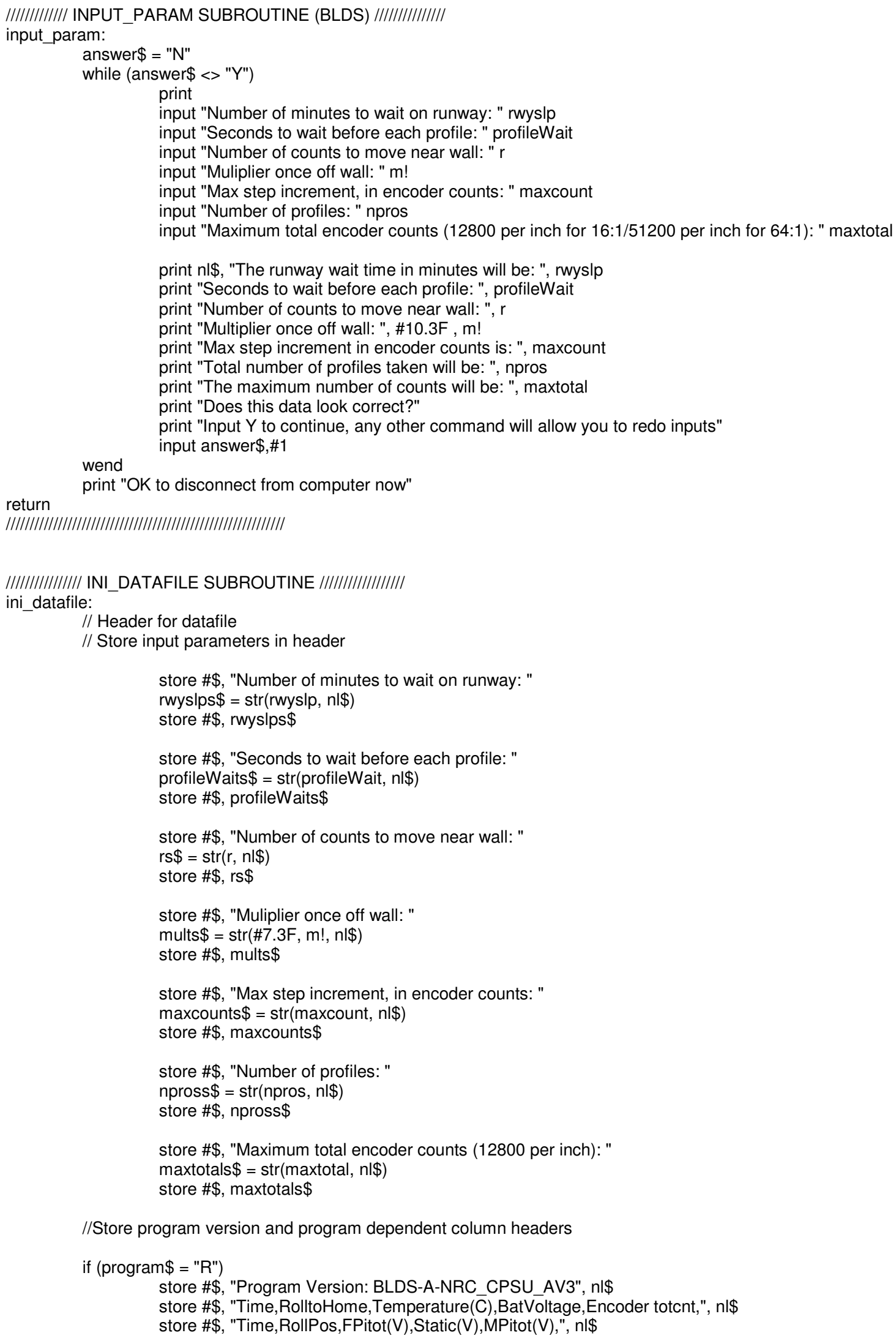




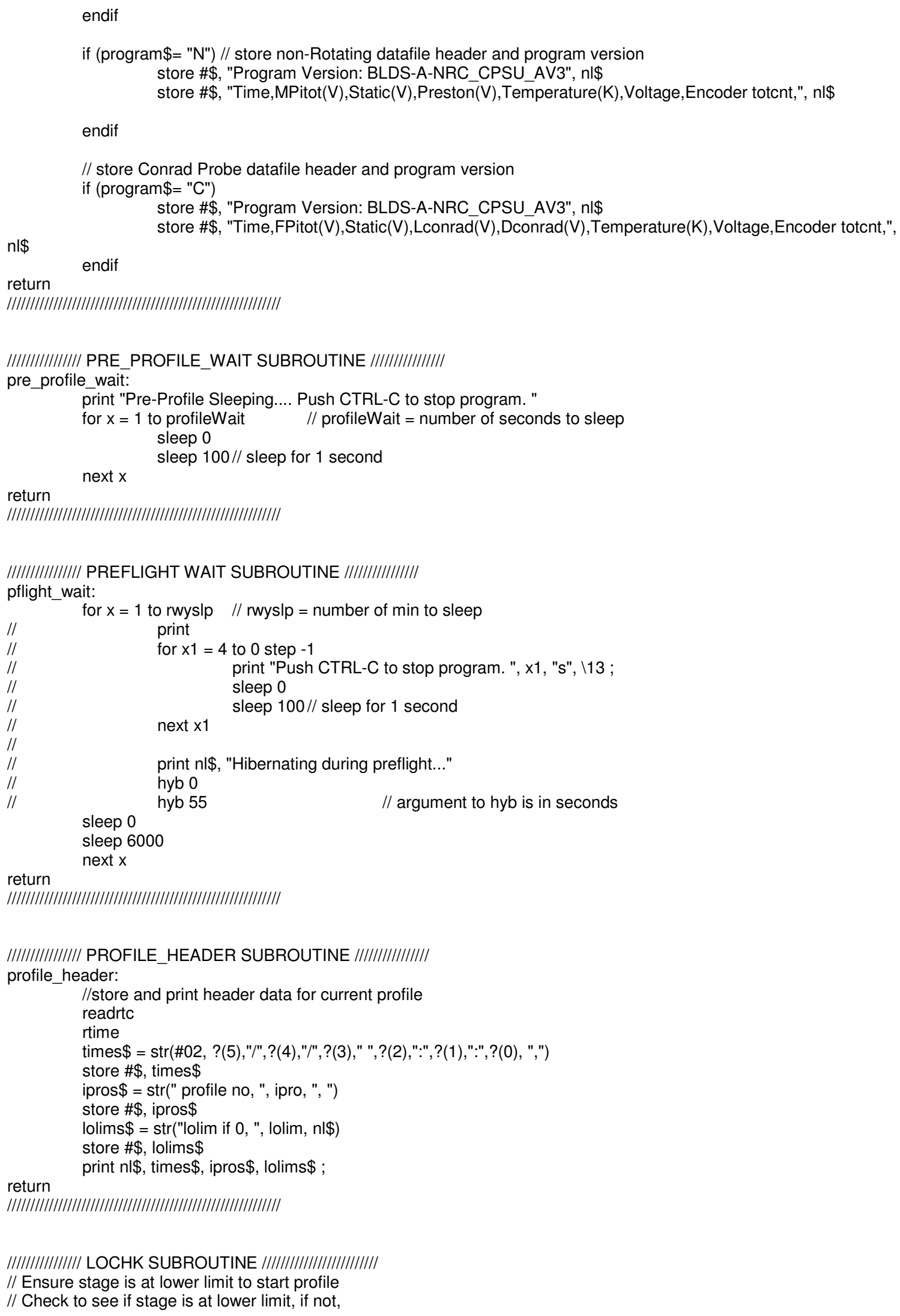


// try to drive twice more at most

// lower limit reached if $P A 6=0$ when $P A 4=1$

// upper limit reached if PA7 $=0$ when $P A 4=0$

lochk:

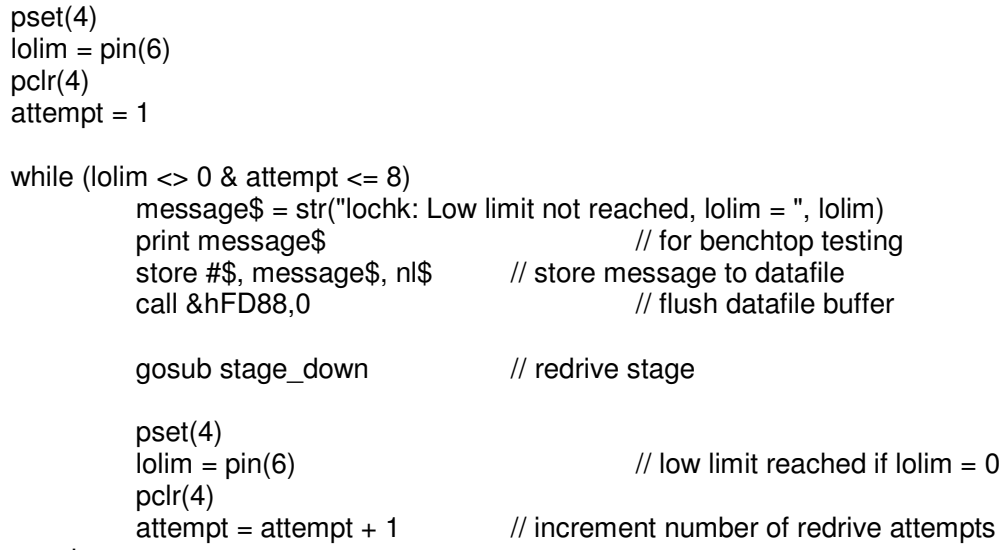

wend

return

|||||||||||||||||||||||||||||||||||||||||||||||||||||||||

|///////////// TAKE PT DATA SUBROUTINE //////////////

take_pt_data:

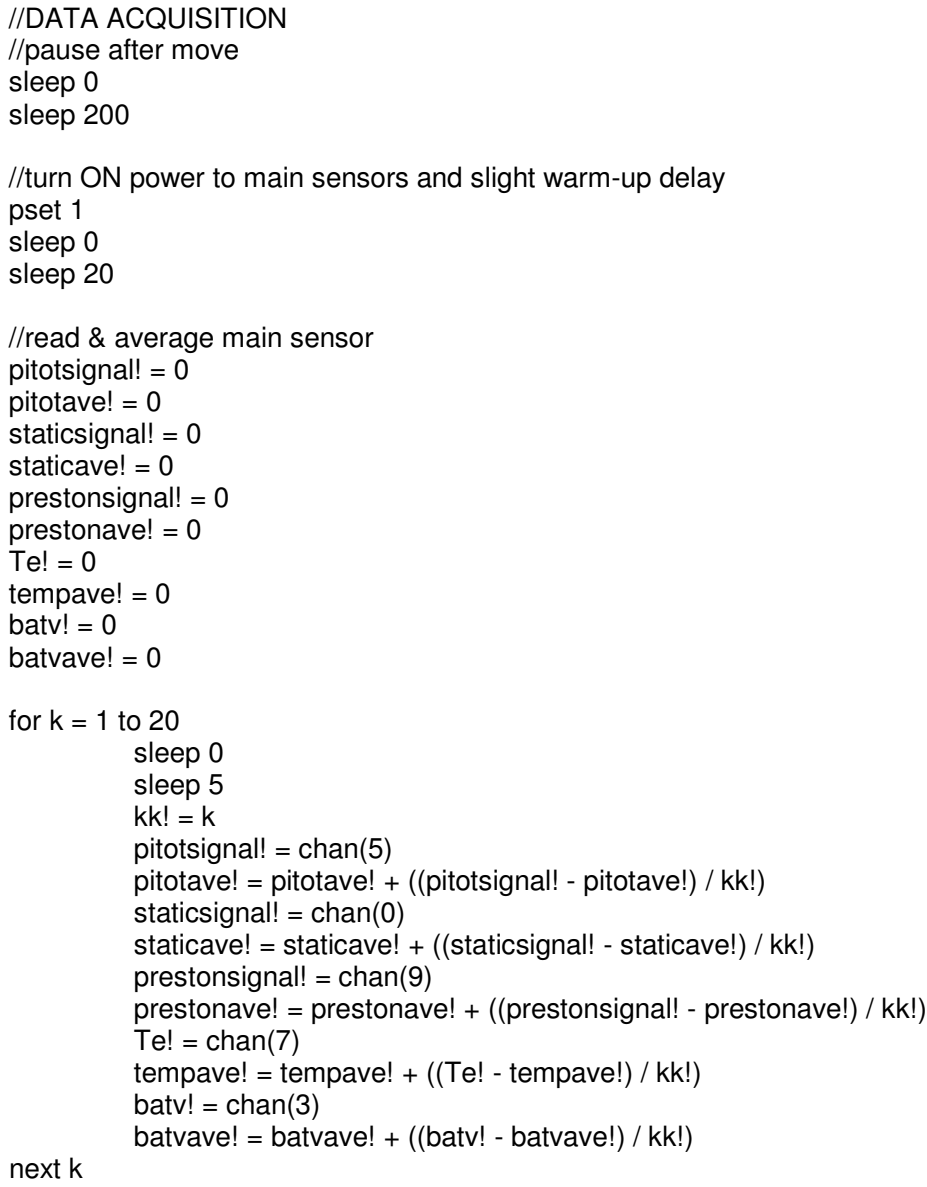




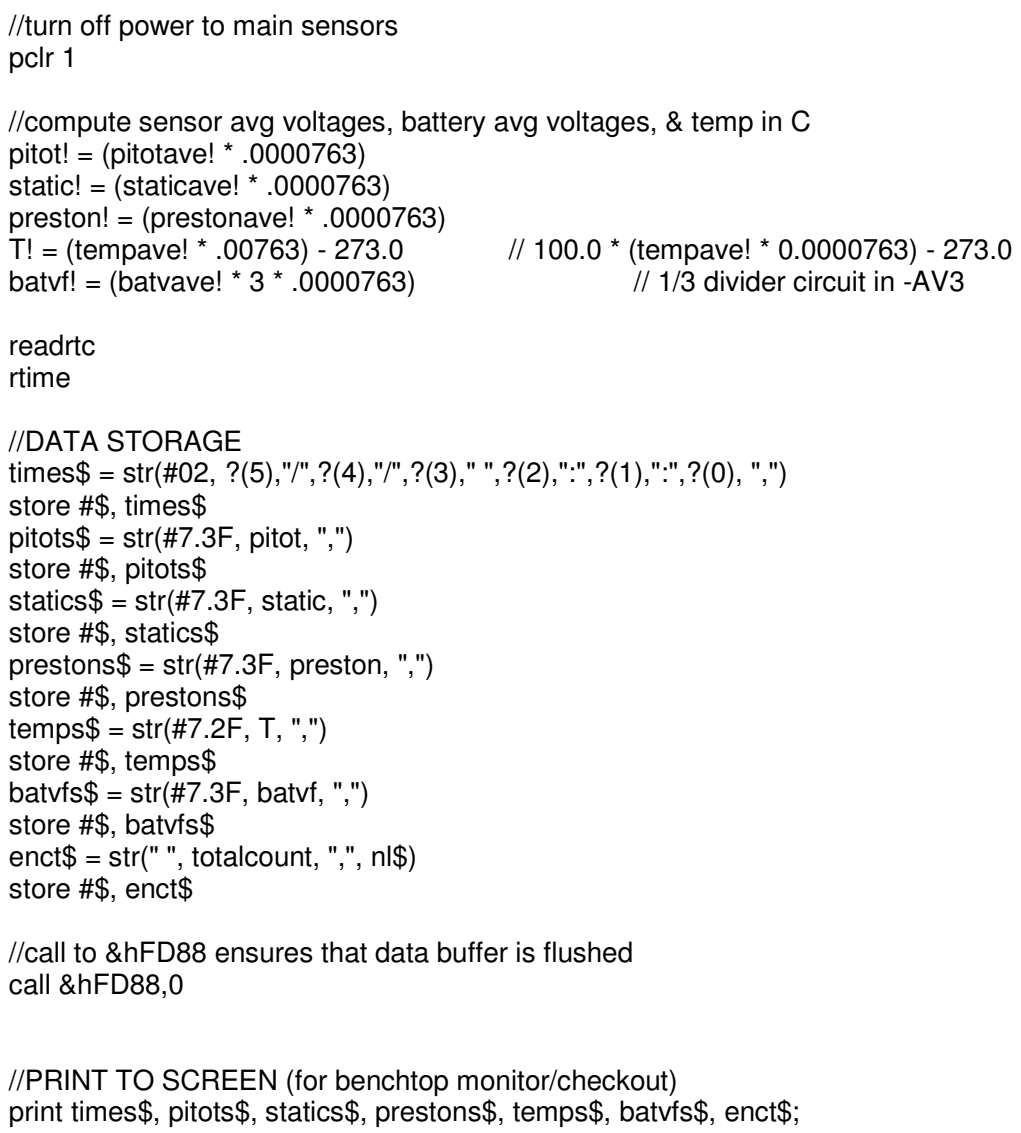

//PRINT TO SCREEN (for benchtop monitor/checkout)

print times $\$$, pitots $\$$, statics $\$$, prestons $\$$, temps $\$$, batvfs $\$$, enct $\$$;

return

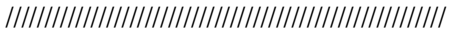

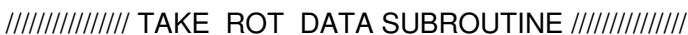

take_rot_data:

// RSP: RECORD SENSOR DATA THEN ROTATE ROLL STEPPER

before -AV3)

// PA4 hi/lo steps the roll motor and RB3 enables step motor and provides hold torque (PA3 was used for this

pclr 4

pset 19

// ensure HOME roll position to start

// rotary home indicator is PA5; check with rotary motor enabled by setting PA3

sleep 0

sleep 20

homect $=0$

homep $=\operatorname{pin}(5)$

while (homep $=0$ \& homect $<24$ )

pset 4

sleep 0

sleep 5

pclr 4

sleep 0

sleep 5

homect $=$ homect +1

wend

homep $=\operatorname{pin}(5)$

//at home position to start--next loop acquires \& records at each roll position

// for $\operatorname{rot}=1$ to 12

for rot $=1$ to 6 


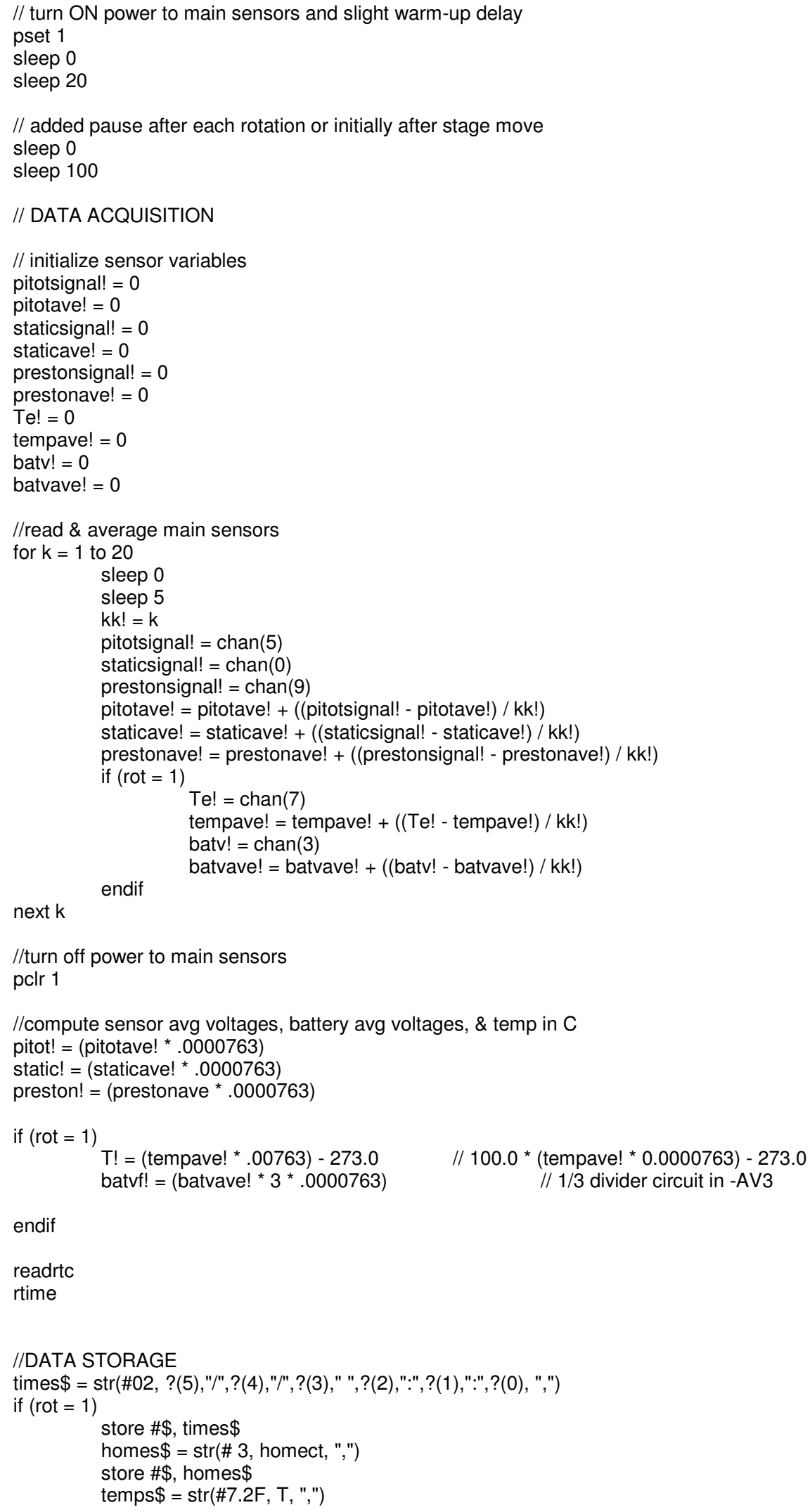


store \#\$, temps\$

batvfs $\$=\operatorname{str}(\# 7.3 F$, batvf, ",")

store \#\$, batvfs $\$$

enct $\$=\operatorname{str}(\# 7$, totalcount, ",", nI\$)

store \#\$, enct $\$$

//PRINT TO SCREEN (for benchtop monitor/checkout)

endif

print $\mathrm{nl} \$$, times $\$$, homes $\$$, temps $\$$, batvfs $\$$, enct $\$$;

store \#\$, times $\$$

rots $\$=\operatorname{str}(\# 3$, rot, ",")

store \#\$, rots $\$$

pitots $\$=\operatorname{str}(\# 7.3 F$, pitot, ",")

store \#\$, pitots $\$$

statics\$ = str(\#7.3F, static, ",")

store \#\$, statics $\$$

prestons $\$=\operatorname{str}(\# 7.3 F$, preston, ",", nI\$)

store \#\$, prestons $\$$

//PRINT TO SCREEN (for benchtop monitor/checkout)

print times $\$$, rots $\$$, pitots $\$$, statics $\$$, prestons $\$$;

//call to \&hFD88 ensures that data buffer is flushed to FLASH EEPROM

call \&hFD 88,0

// rotate to next position

// RB3 enables step motor; PA4 hi/lo steps

$/ /$ roll motor is 24 steps/rev; $24 /$ nrsteps = number of rolls per stage location

$/ /$ nrsteps ${ }^{*} 15$ deg $=$ degrees between roll steps

// set nrsteps to 1 gives 24 rolls per stage location (15 deg increments)

$/ /$ nrsteps $=2$ gives 30 deg between steps, 12 roll locations

$/ /$ nrsteps $=3$ gives 45 deg between steps, 8 roll locations (normally used in flight before TAMU)

$/ /$ nrsteps $=4$ gives 60 deg between steps, 6 roll locations (used for TAMU flights)

$/ /$ nrsteps $=6$ gives 90 deg between steps, 4 roll locations (not yet tried)

nrsteps $=4$

for rstep $=1$ to nrsteps

pset(4)

sleep 0

sleep 5

$\operatorname{pclr}(4)$

sleep 0

next rstep

next rot

sleep 5

// Clear RB3 to turn off stepper motor--no hold torque now!

pclr 19

pclr 4

return

||||||||||||||||||||||||||||||||||||||||||||||||||||||||

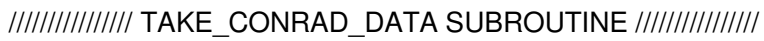

take conrad data:

//DATA ACQUISITION

//pause after move

sleep 0

sleep 200

//turn ON power to main sensors and additional conrad sensor and slight warm-up delay

pset 1

pset 3

sleep 0

sleep 20

//read \& average main sensor

Fpitotsignal! $=0$

Fpitotave $=0$ 


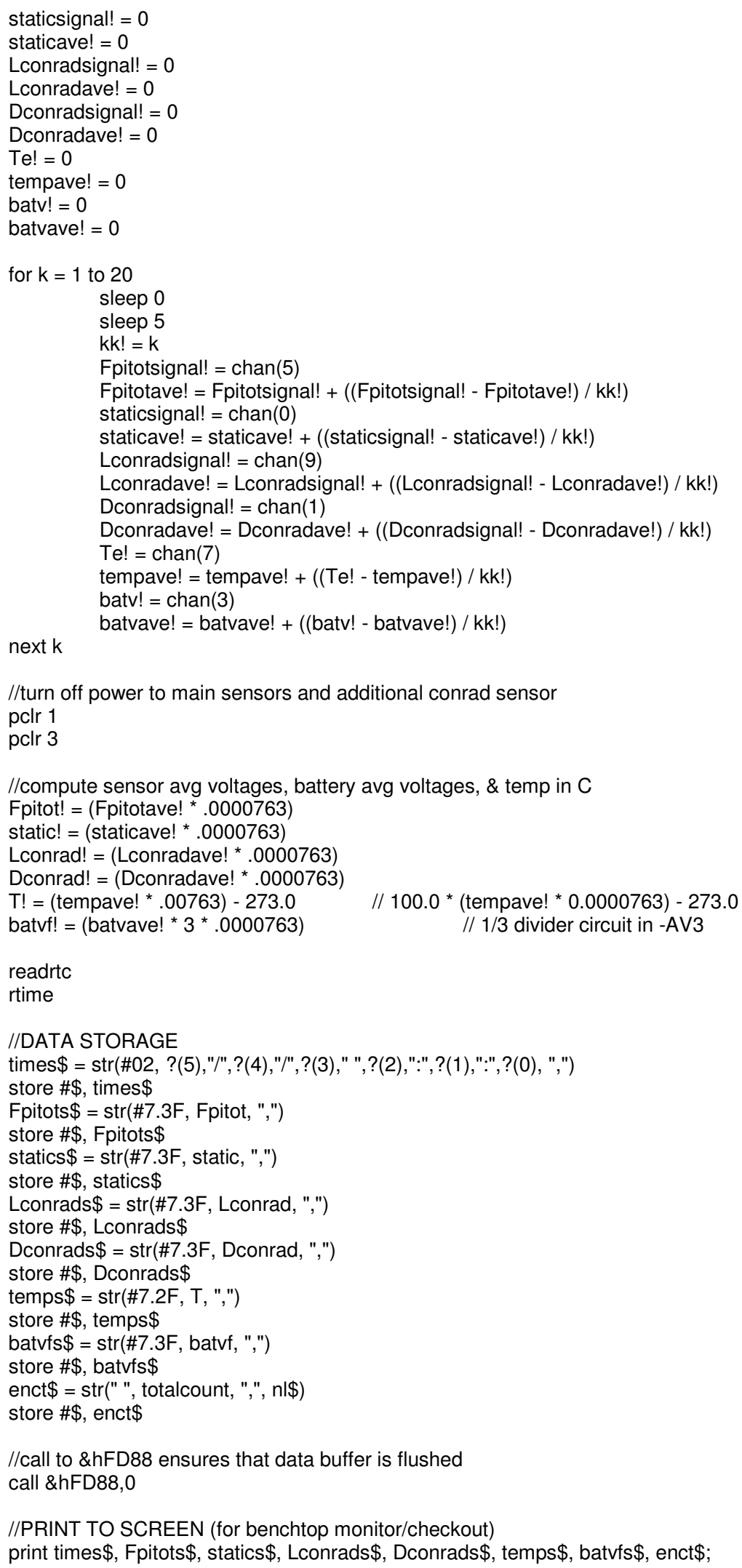

//PRINT TO SCREEN (for benchtop monitor/checkout)

print times\$, Fpitots\$, statics\$, Lconrads\$, Dconrads\$, temps\$, batvfs\$, enct\$; 


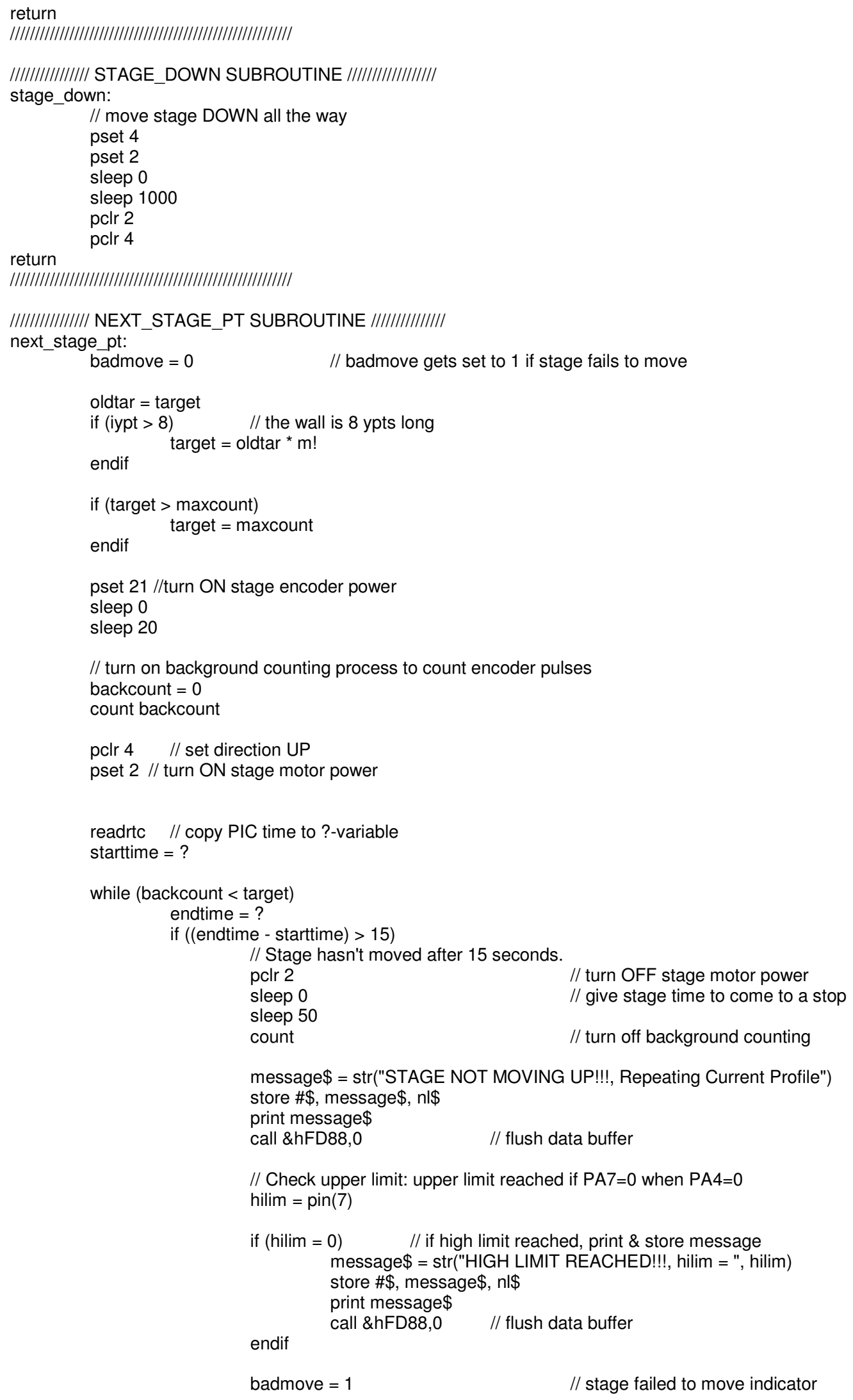




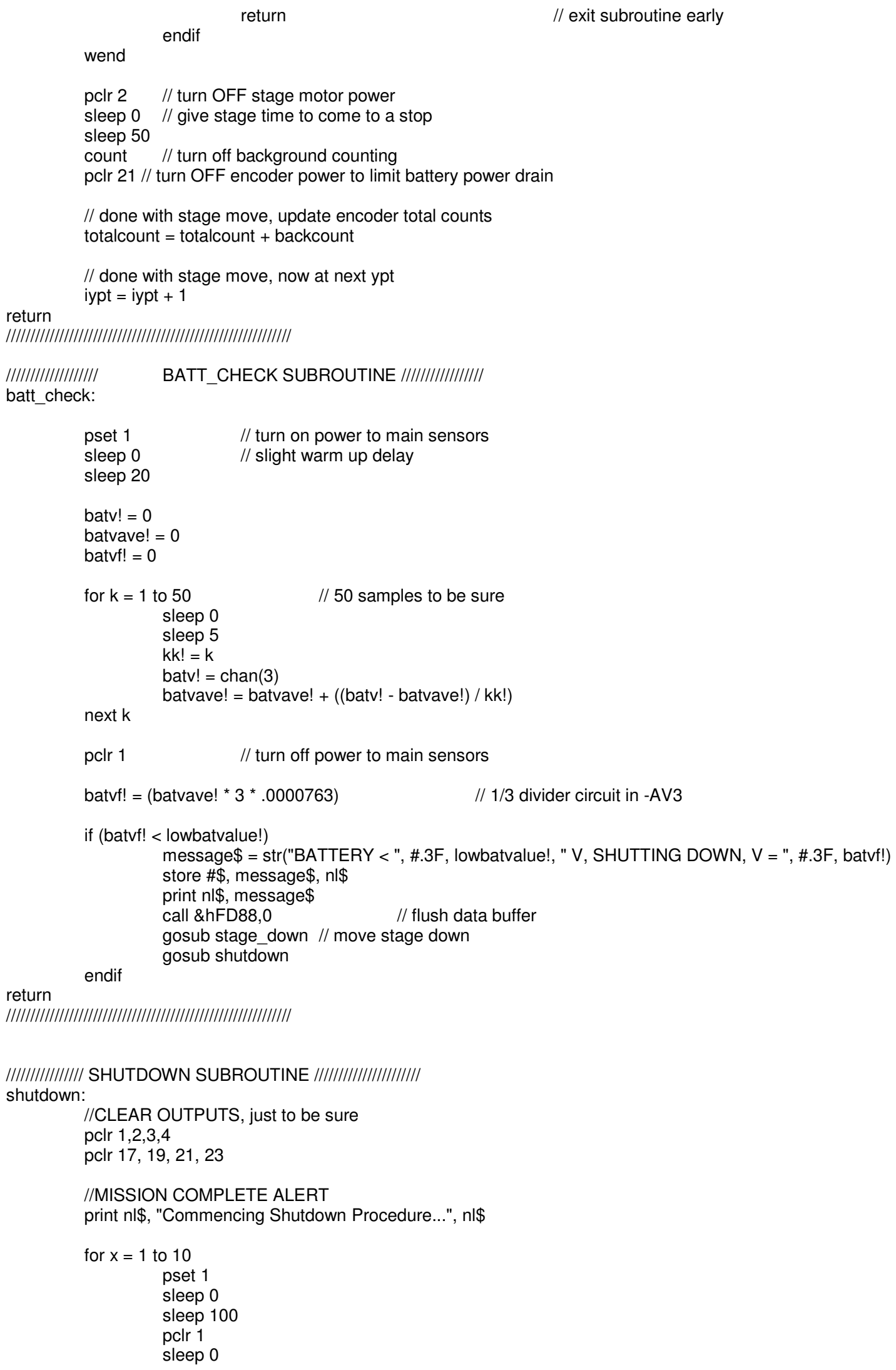




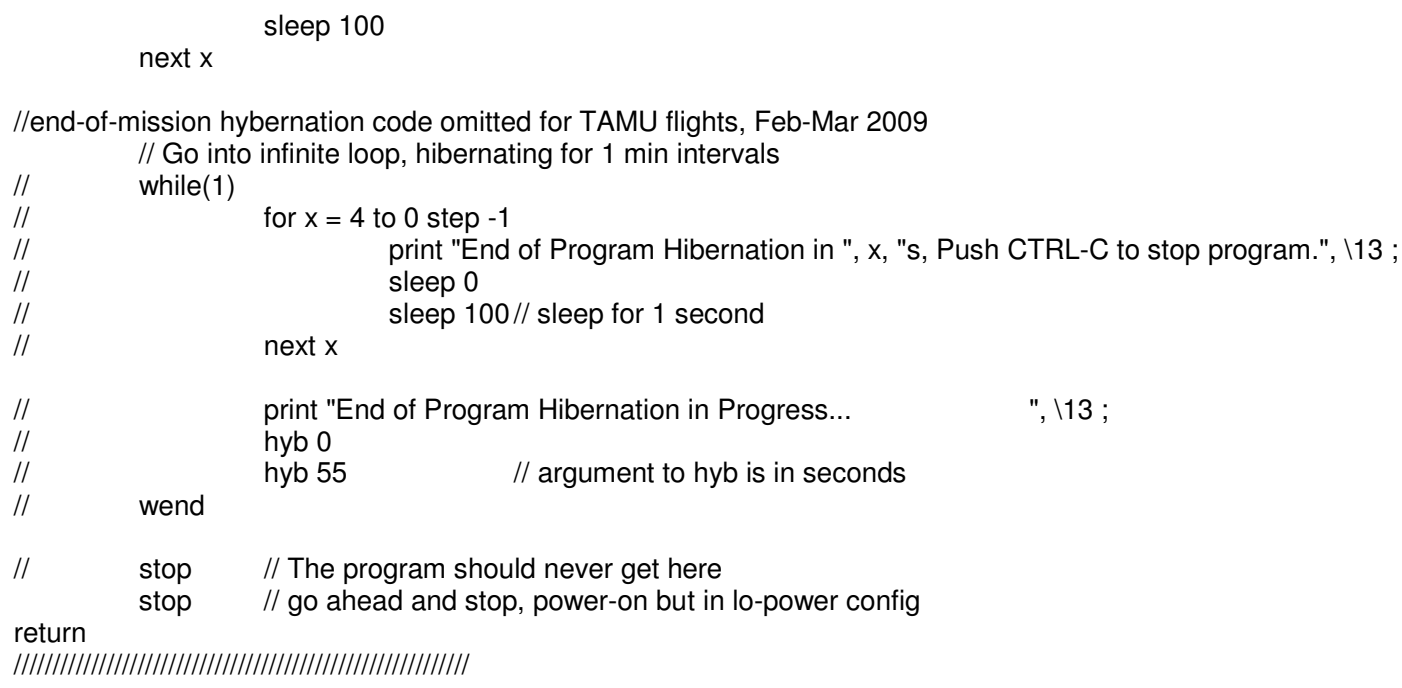


APPENDIX B: Drawings of Conrad Probe

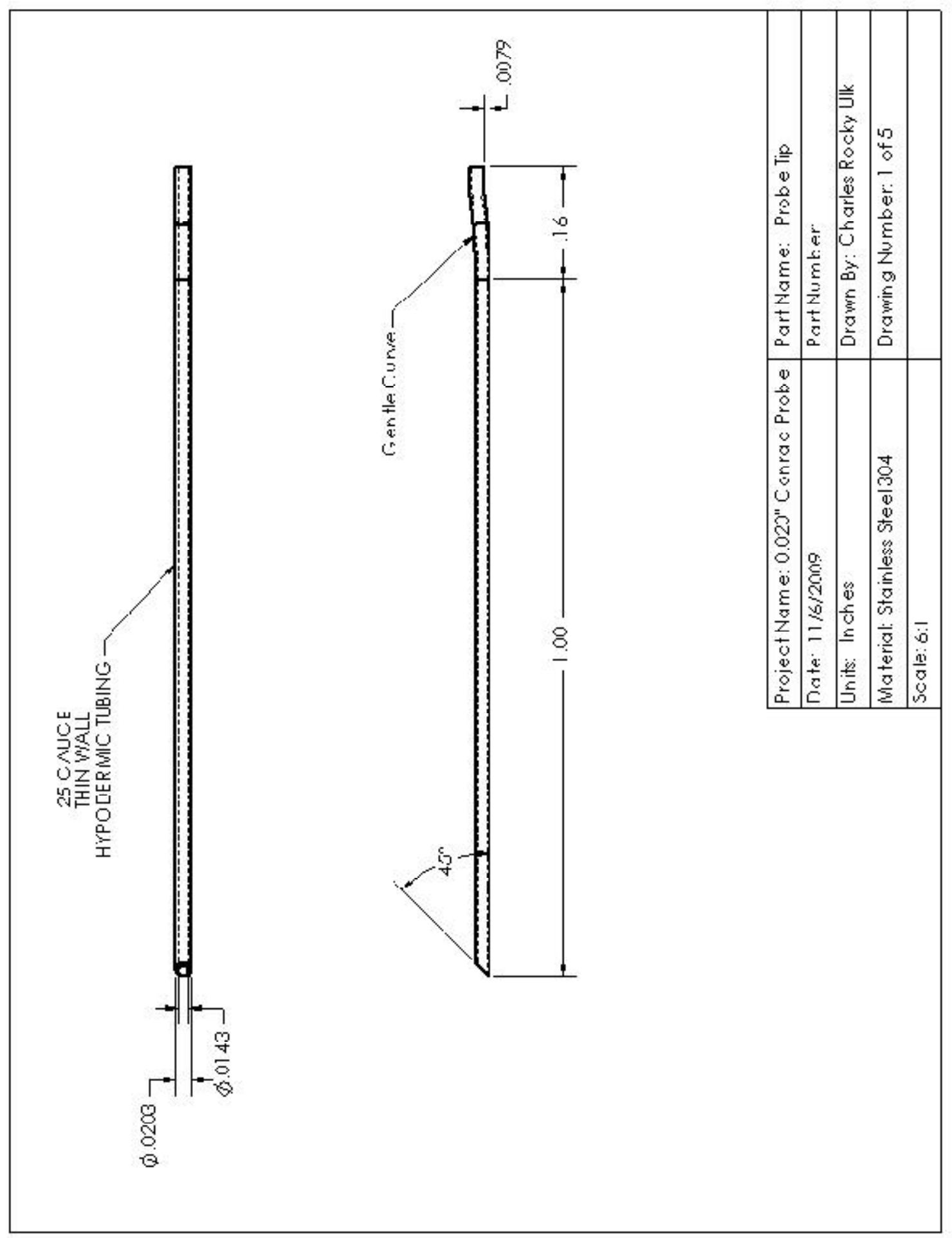



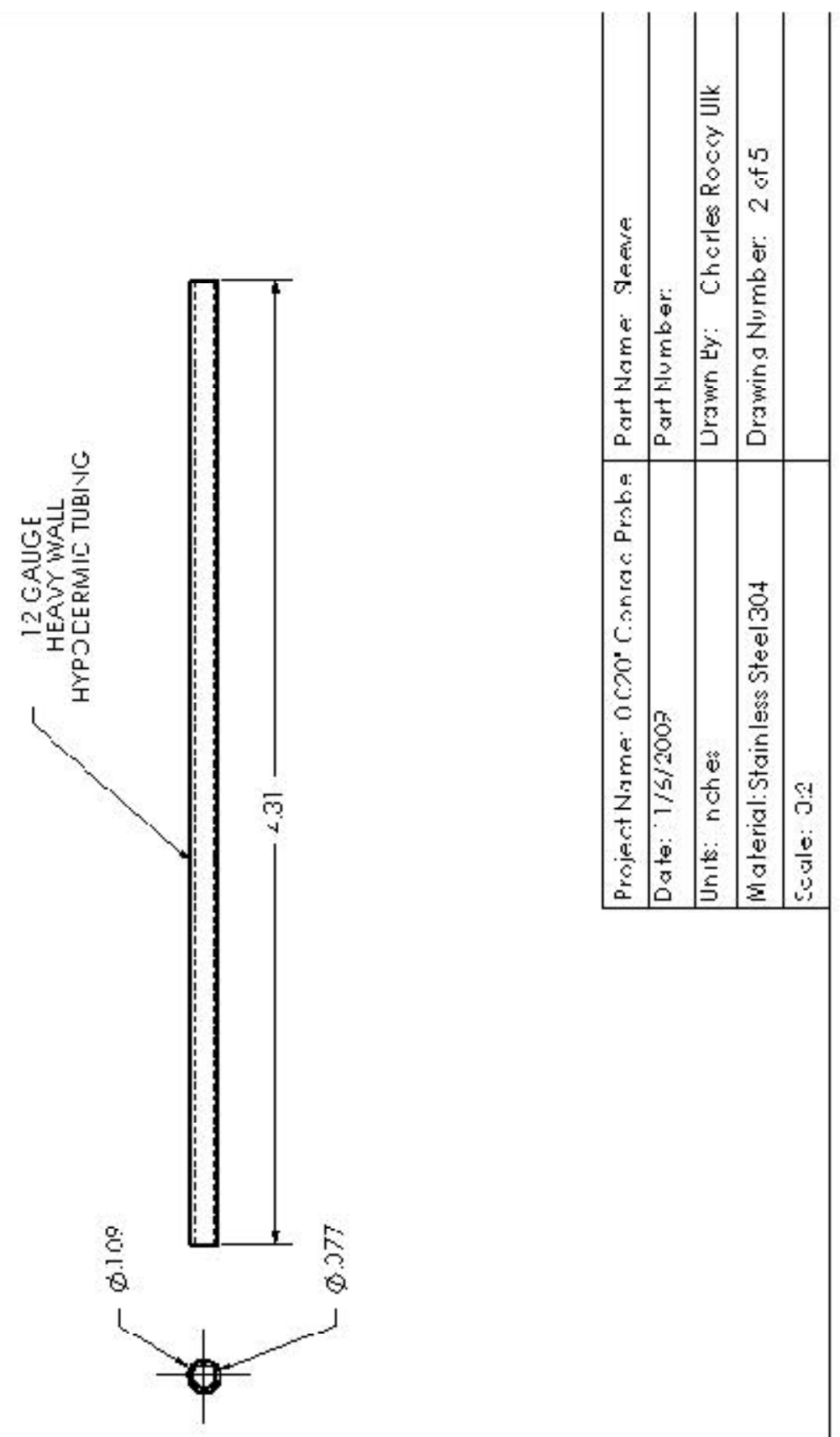


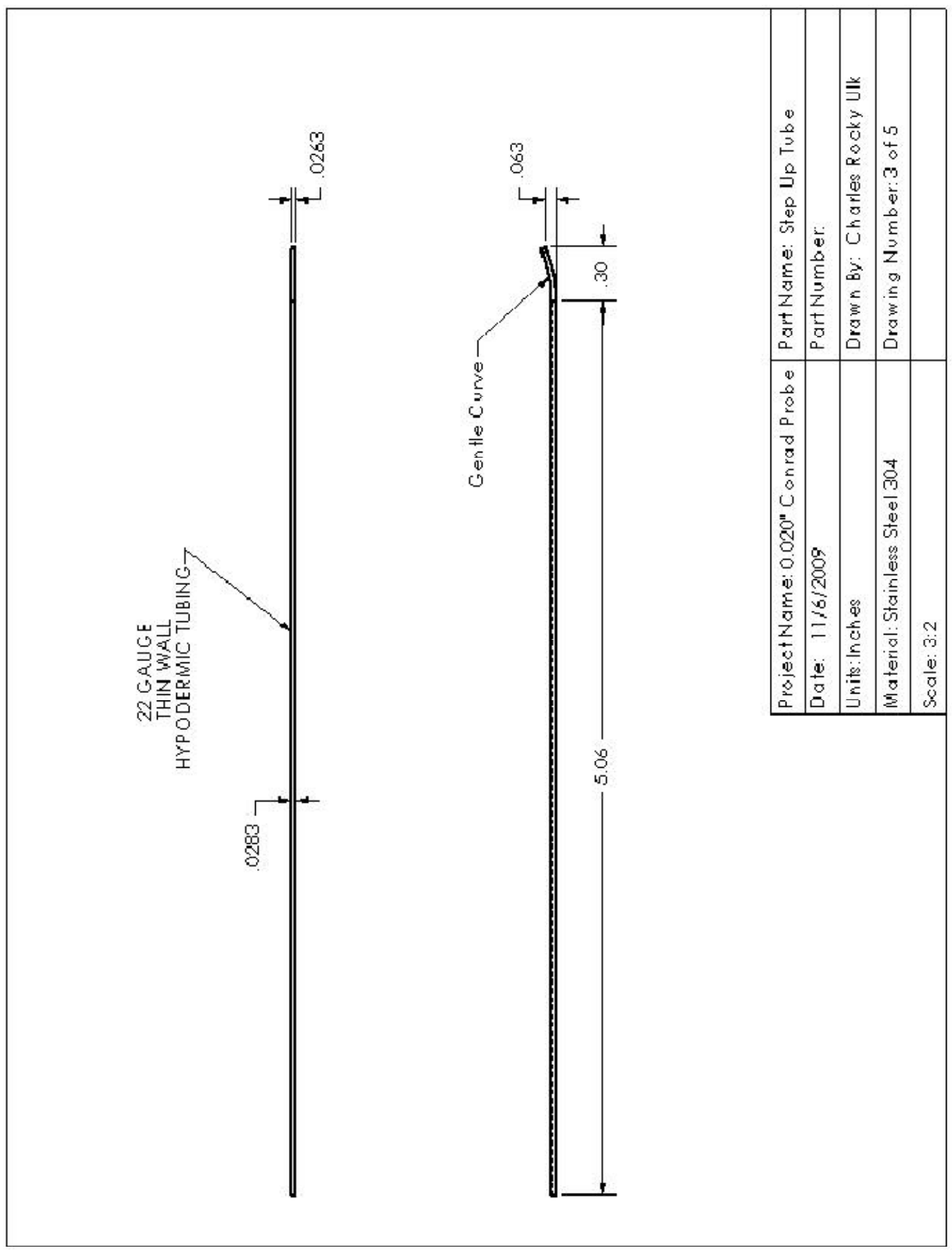




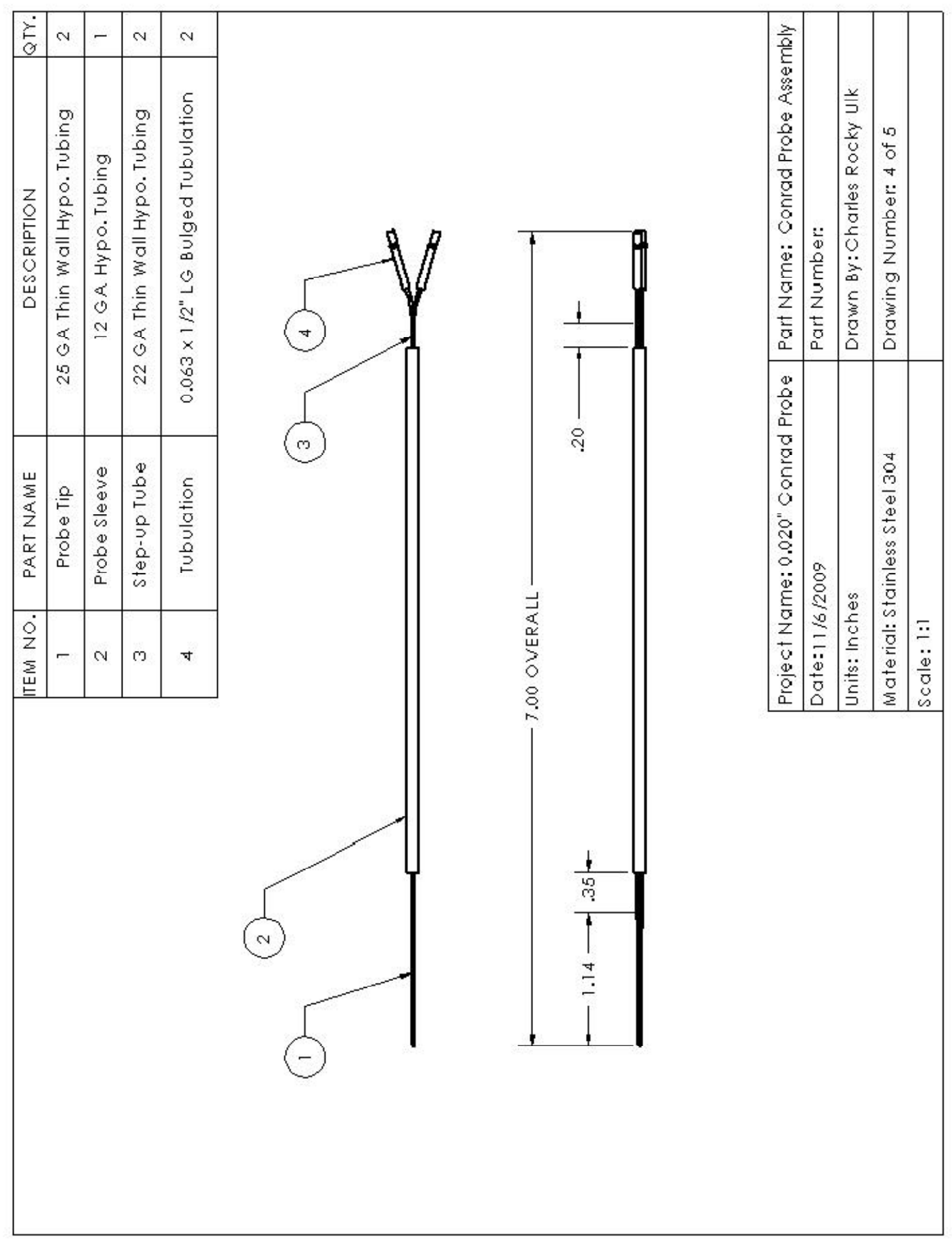




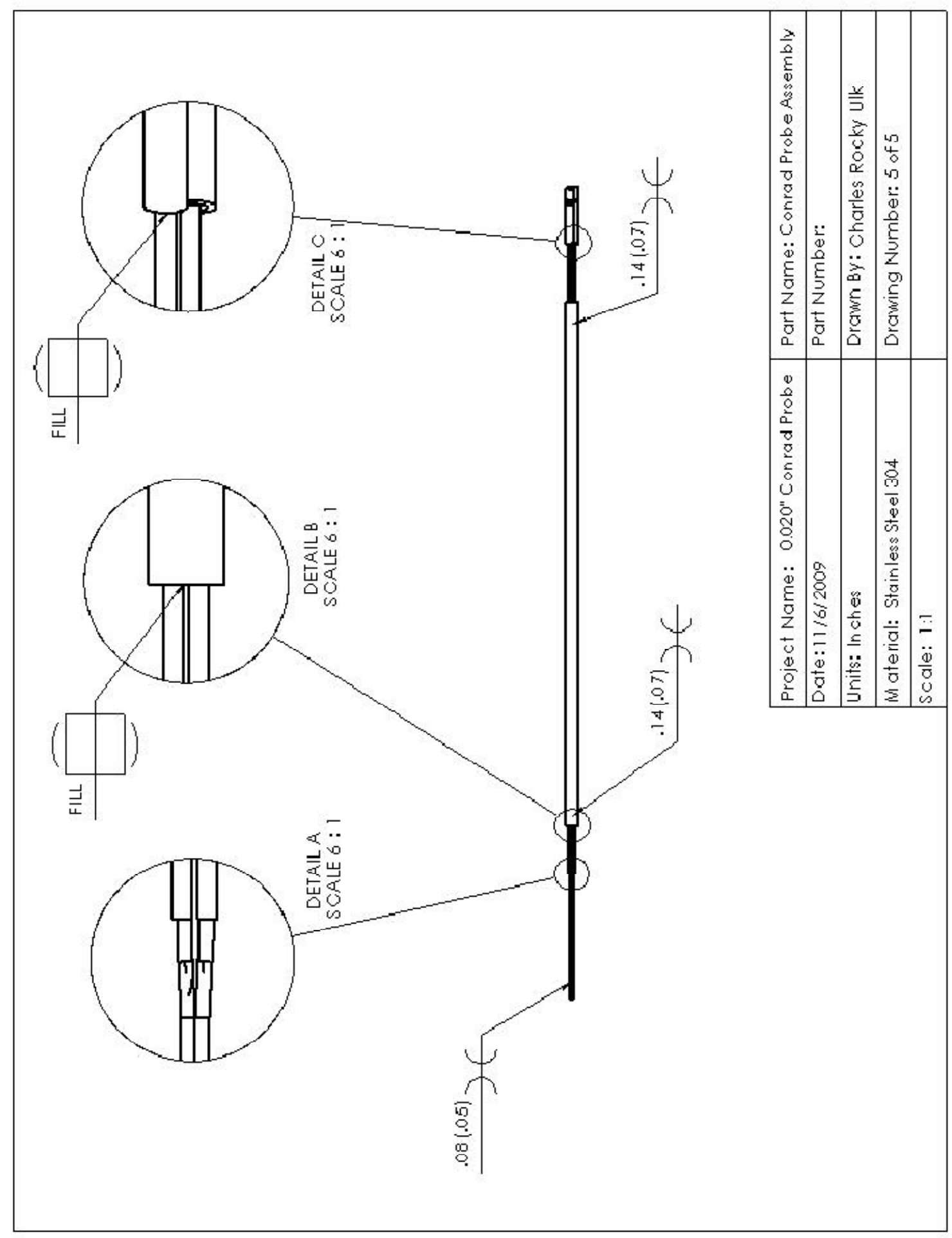




\section{APPENDIX C: Secondary Flow Calculation for Swept Flat Plate}

The treatment of three-dimensional boundary layers over a swept flat plate can be approximated by the calculation of secondary flow over a yawed infinite cylinder. The calculation is brought about by reduction of the Navier-Stokes equations. A full procedure is detailed by W.R. Sears in Reference 27 and again by Rosenhead in Reference 28 . The starting equations are shown below using Einstein's notation:

$$
\rho \frac{D u}{D t}=\rho g-\nabla p+\frac{\partial}{\partial x_{j}}\left[\mu\left(\frac{\partial v_{i}}{\partial x_{j}}+\frac{\partial v_{j}}{\partial x_{i}}\right)+\delta_{i j} \lambda \operatorname{div} V\right]
$$

Assumptions are made about the flow behavior to reduce them to

$$
\begin{gathered}
u \frac{\partial u}{\partial x}+w \frac{\partial u}{\partial z}=U \frac{d U}{d x}+v \frac{\partial^{2} u}{\partial z^{2}} \\
u \frac{\partial v}{\partial x}+w \frac{\partial v}{\partial z}=v \frac{\partial^{2} v}{\partial z^{2}} \\
\frac{\partial u}{\partial x}+\frac{\partial w}{\partial z}=0
\end{gathered}
$$

with the boundary conditions

$$
\begin{gathered}
u=v=w=0 \text { when } z=0, \\
u \rightarrow U, v \rightarrow V \text { as } z \rightarrow \infty .
\end{gathered}
$$

The above equations exhibit a property known as the independence principle, where two equations can be solved independently of the third. Prantl 
gave a solution to this set of equations for flow in near a stagnation line of which applies a non-dimensional parameter $\eta$, defined as;

$$
\eta=\frac{R e^{\frac{1}{2}} Z}{L}
$$

where $R e$ is the Reynolds number, $z$ is the height above the surface, and $L$ is the characteristic length. Using eta, the equations further reduce to a set of ordinary differential equations, which can be solved in the usual way. The result of solving these equations is a solution for the behavior of a boundary layer in two dimensions. Figures C.1 and C.2 below, which show the shape of the boundary layers broken into components and non-dimensionalized [28].

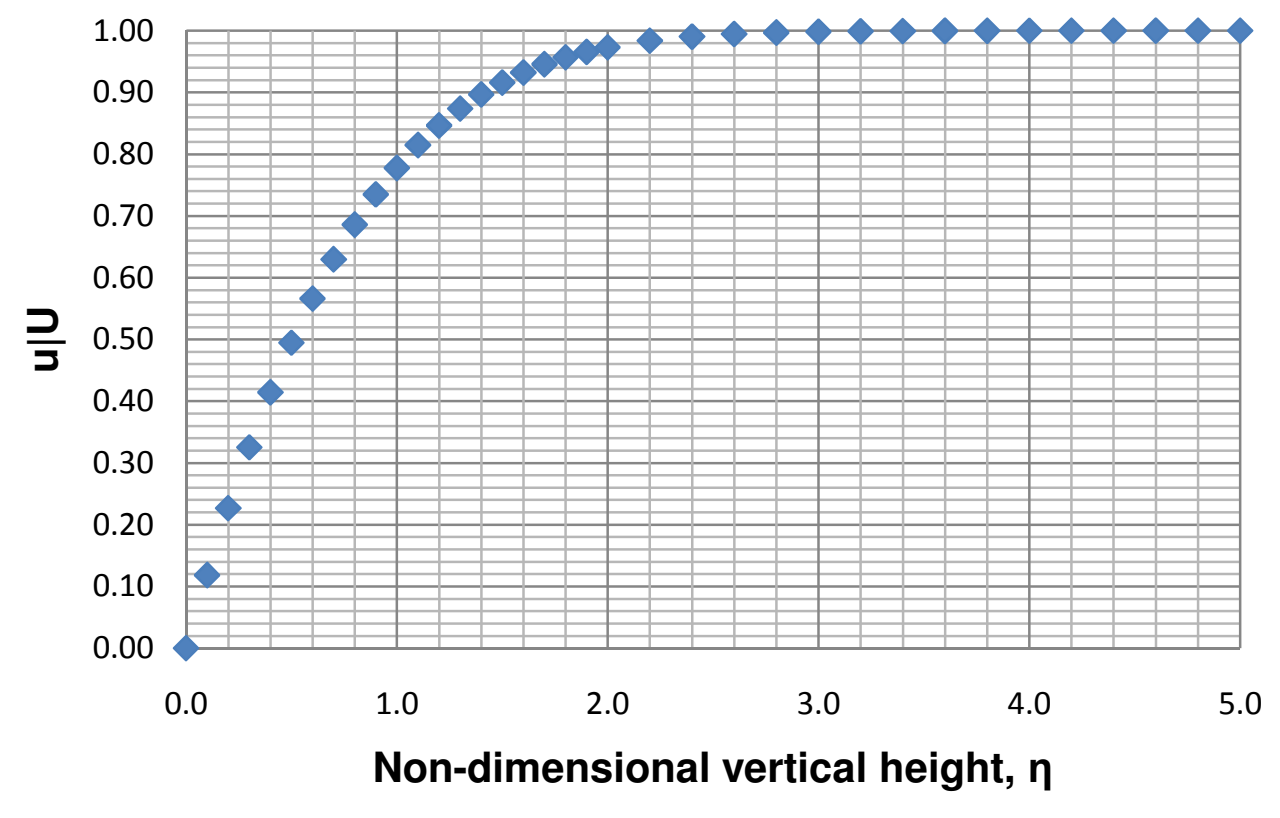

Figure $\mathrm{C.1}-\mathrm{u} \mid \mathrm{U}$ vs. height for cylinder in yawed flow 


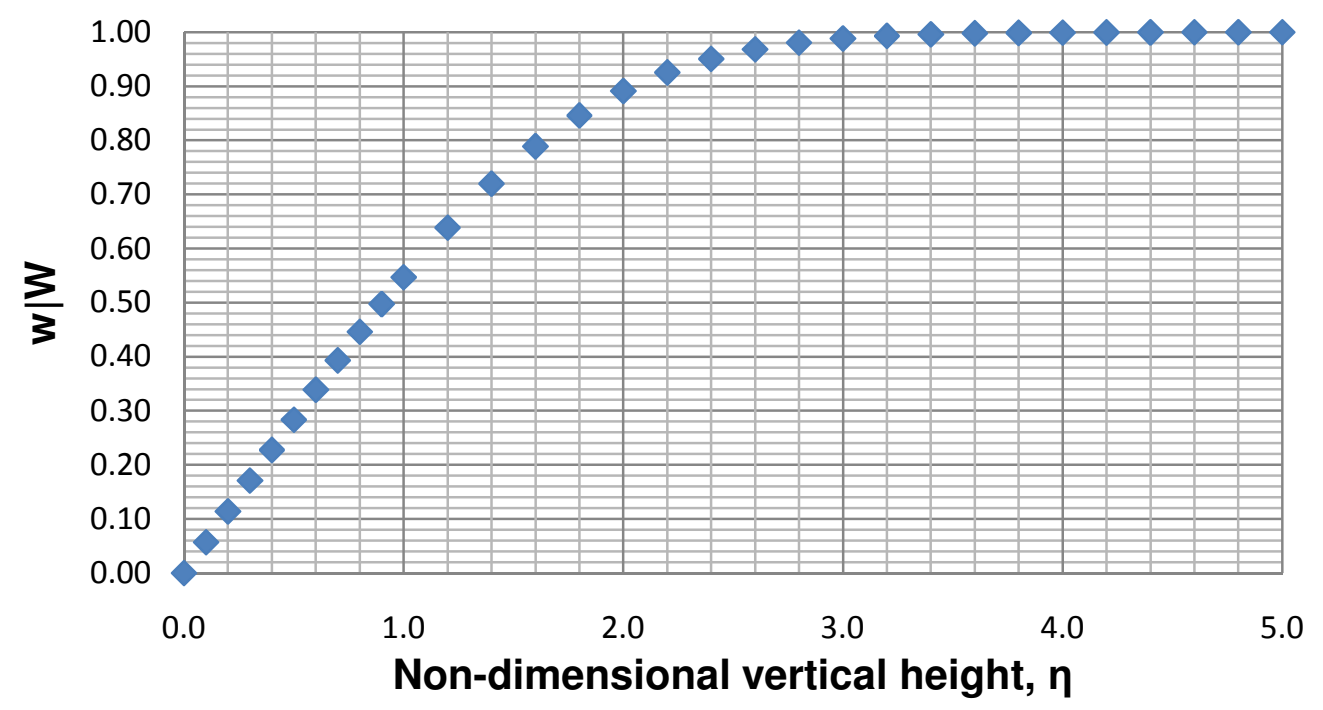

Figure $\mathbf{C} .2-\mathrm{w} \mid \mathrm{W}$ vs. height for cylinder in yawed flow

The graphs both show the behavior of the solution as the height above a surface is increased. To be useful in analyzing the validity of the swept flat plate for use, a series of calculations of the $\mathrm{U}$ and $\mathrm{W}$ components of velocity aft of the leading edge was performed under the conditions listed in Table C.1.

Table C.1 - Parameters for analysis of swept flat plate using Sears' Solution

$\begin{array}{llrl}\text { Lead Edge Radius, } \mathbf{r}_{\mathrm{le}} & & 3 \mathrm{~mm} \\ \text { Free-stream Velocity } & = & 110 \mathrm{mph} \\ \text { Plate Sweep Angle } & = & 30 \mathrm{degrees} \\ \text { Density } & = & 1.3 \mathrm{~kg} / \mathrm{m}^{3} \\ \text { Viscosity } & = & 1.83 \mathrm{e}-05 \mathrm{~kg} / \mathrm{m} \mathrm{s} \\ \text { Reference length, L } & 3 \mathrm{inches} \\ \text { Distance from leading edge, } \mathbf{x} & = & \text { VARIOUS } \mathrm{m}\end{array}$

Along with the above parameters, a 2-dimensional smooth cylindrical leading edge approximation was used in the calculation of the stagnation point velocity gradient parameter $\mathrm{B}$.

$$
B=\frac{4 q_{R E F}}{r_{l e}}
$$


With the solvable simplified Navier-Stokes equations and the stagnation gradient parameter found, the solution could be computed at various distances from the leading edge of the simulated plate. For this very specific case the calculation was used to establish that secondary flow would be generated by the sweep angle on the plate. As can be seen from Figure C.3 a change in the yaw angle $\beta$ from the nominal free-stream direction was found to occur close to the leading edge of the plate and continue downstream with the flow angle change slowly decreasing.

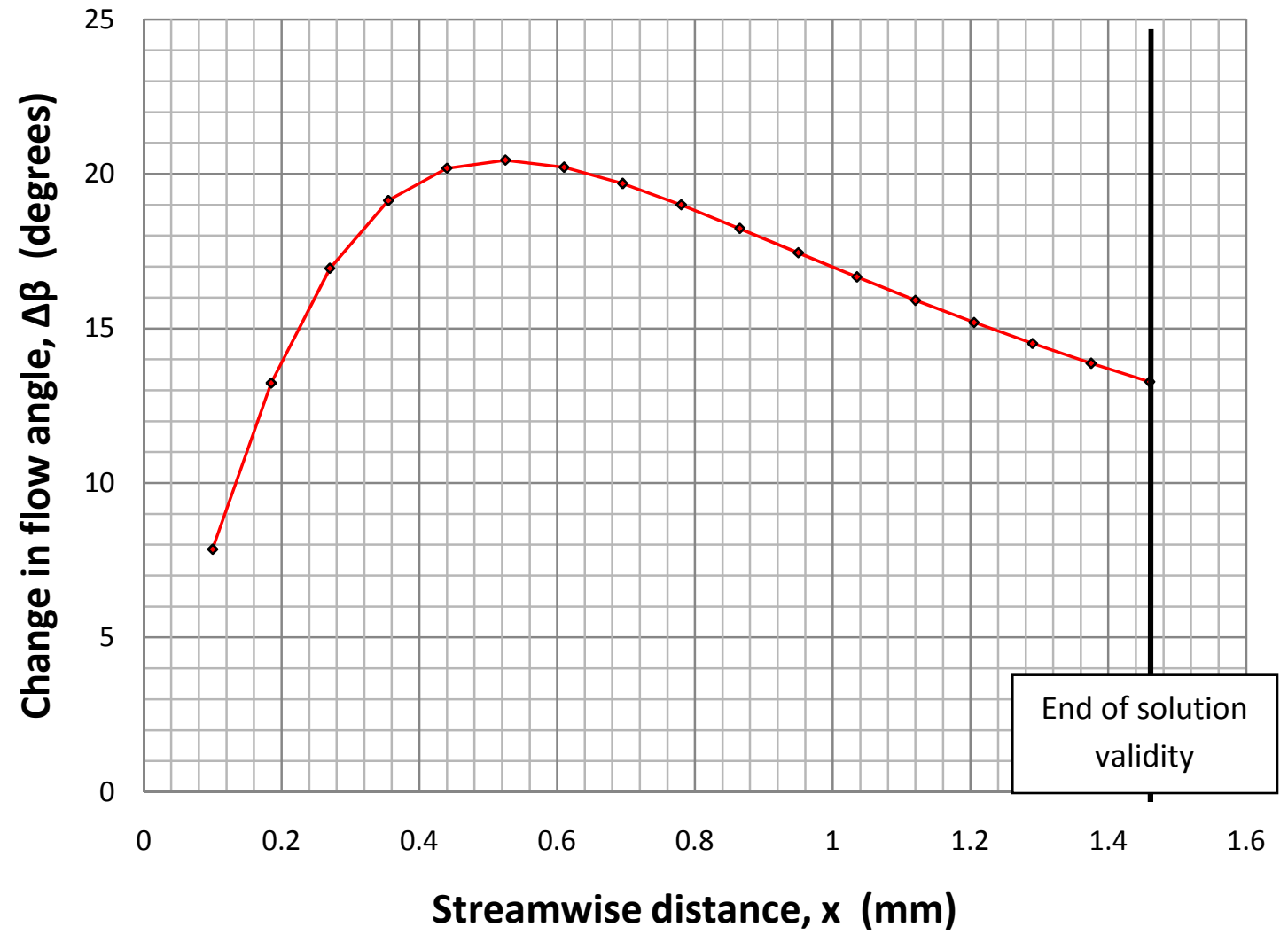

Figure C.3 - Maximum flow angle change versus stream-wise $(x)$ distance from the leading edge 
APPENDIX D: Uncertainty Analysis for the Conrad Probe on the BLDS Unit

The BLDS unit measures three pressures, $P_{L}, P_{L-R}$, and $q_{R E F}$ to compute two unknown quantities, $\beta$ and $\frac{q}{q_{R E F}}$. These measurements provide a source of uncertainty to any calculations involving their use and as such, it became necessary to quantify the uncertainty involved with them. A method of error estimation, called sequential perturbation was used to quantify these uncertainties. In this method the propagation of uncertainty in a set of measurements is estimated using a finite difference approximation of the partial derivatives of the computed quantities with respect to measured quantities. In other words, the measured quantities are "perturbed" individually by an amount equal to the uncertainty [29][30].

Measured Quantities: $\quad x_{1}=P_{L} ; \quad x_{2}=P_{L-R} ; x_{3}=q_{R E F}$

The estimated values for the uncertainties, $u_{s}$ were reduced from the manufacturer quoted $1 \%$ FS value to a value of $0.25 \%$ FS based upon previous experience with other measurement schemes, the elimination of zero drift error with use of wind-off zeros, and the fact that only $15 \%$ of the usable range of the pressure sensors was being used for the Conrad probe measurement scheme. 
Uncertainties: $\quad u_{x 1}=( \pm 0.0025 * 1.5$ sid $)=0.00375$ psid

$$
\begin{aligned}
& u_{x 2}=( \pm 0.0025 * 3.0 \text { psid })=0.0075 \text { psid } \\
& u_{x 3}=( \pm 0.0025 * 1.5 \text { psid })=0.00375 \text { psid }
\end{aligned}
$$

After the measured quantities are perturbed by the uncertainties, the calculation of the computed quantities is carried out for each perturbed measured quantity.

Computed Quantities: $\quad \beta\left(\mathrm{x}_{1}, \mathrm{x}_{2}, \mathrm{x}_{3}\right)$ and $\frac{q}{q_{R E F}}\left(\mathrm{x}_{1}, \mathrm{x}_{2}, \mathrm{x}_{3}\right)$

Pert.Computed Quantities: $\beta\left(x_{1}+u_{x 1}, x_{2}, x_{3}\right) ; \beta\left(x_{1}, x_{2}+u_{x 2}, x_{3}\right) ; \beta\left(x_{1}, x_{2}, x_{3}+u_{x 3}\right)$

$$
\frac{q}{q_{R E F}}\left(\mathrm{x}_{1}+\mathrm{U}_{\mathrm{x} 1}, \mathrm{x}_{2}, \mathrm{x}_{3}\right) ; \frac{q}{q_{R E F}}\left(\mathrm{x}_{1}, \mathrm{x}_{2}+\mathrm{U}_{\mathrm{x} 2}, \mathrm{x}_{3}\right) ; \frac{q}{q_{R E F}}\left(\mathrm{x}_{1}, \mathrm{x}_{2}, \mathrm{x}_{3}+\mathrm{U}_{\mathrm{x} 3}\right)
$$

The collective errors obtained from each perturbed quantity are then averaged with the root mean square method and an estimation of the total error for each computed quantity is found:

Differences*: $\Delta x_{1}=\beta\left(x_{1}+u_{x 1}, x_{2}, x_{3}\right)-\beta\left(x_{1}, x_{2}, x_{3}\right)$

$$
\begin{aligned}
& \Delta x_{2}=\beta\left(x_{1}, x_{2}+u_{x 2}, x_{3}\right)-\beta\left(x_{1}, x_{2}, x_{3}\right) \\
& \Delta x_{3}=\beta\left(x_{1}, x_{2}, x_{3}+u_{x 3}\right)-\beta\left(x_{1}, x_{2}, x_{3}\right)
\end{aligned}
$$

Total Propagated Uncertainty*: $u_{\beta}=\sqrt{\Delta x_{1}^{2}+\Delta x_{2}^{2}+\Delta x_{3}^{2}}$

${ }^{*}$ Calculation is the same for $\frac{q}{q_{R E F}}$ 
The total propagated uncertainty was computed on one data set from

results obtained by the BLDS at $x \mid c=0.25$ on the flat plate in the $2^{\prime} \times 2$ ' wind

tunnel. The results are shown in Tables D.1 and D.2 below.

Table D.1 $-\beta$ uncertainty results from flat plate data

\begin{tabular}{|c|c|c|c|c|c|c|c|c|c|c|}
\hline$x 1\left(P_{L}\right)$ & X2 $\left(P_{L-R}\right)$ & x3 (q $\left.q_{\text {REF }}\right)$ & $\beta$ & $\beta\left(x_{1}+u_{x_{1}}\right)$ & $\beta\left(x_{1}+u_{x_{1}}\right)$ & $\beta\left(x_{1}+u_{x_{1}}\right)$ & $\Delta \mathrm{x1}$ & $\Delta \times 2$ & $\Delta \times 3$ & $u_{\beta}$ \\
\hline 0.0730 & 0.0495 & 0.2120 & 10.2326 & 9.3523 & 13.0802 & 10.2326 & -0.8802 & 2.8476 & 0.0000 & 2.9806 \\
\hline 0.0767 & 0.0495 & 0.2127 & 9.3513 & 8.5693 & 11.9999 & 9.3514 & -0.7821 & 2.6485 & 0.0000 & 2.7616 \\
\hline 0.0808 & 0.0495 & 0.2119 & 8.4946 & 7.8031 & 10.9521 & 8.4945 & -0.6915 & 2.4575 & 0.0000 & 2.5529 \\
\hline 0.0778 & 0.0495 & 0.2116 & 9.1065 & 8.3508 & 11.7003 & 9.1065 & -0.7557 & 2.5938 & 0.0000 & 2.7017 \\
\hline 0.0876 & 0.0540 & 0.2153 & 8.6032 & 7.9562 & 10.8721 & 8.6031 & -0.6469 & 2.2689 & 0.0000 & 2.3593 \\
\hline 0.1067 & 0.0593 & 0.2127 & 7.0547 & 6.5916 & 8.8347 & 7.0547 & -0.4631 & 1.7800 & 0.0000 & 1.8392 \\
\hline 0.1101 & 0.0645 & 0.2120 & 7.8225 & 7.3383 & 9.5820 & 7.8225 & -0.4842 & 1.7595 & 0.0000 & 1.8250 \\
\hline 0.1153 & 0.0698 & 0.2142 & 8.3009 & 7.8170 & 9.9995 & 8.3009 & -0.4839 & 1.6986 & 0.0000 & 1.7662 \\
\hline 0.1176 & 0.0743 & 0.2127 & 8.9896 & 8.4849 & 10.6839 & 8.9896 & -0.5047 & 1.6943 & 0.0000 & 1.7679 \\
\hline 0.1311 & 0.0788 & 0.2138 & 8.1979 & 7.7741 & 9.6847 & 8.1979 & -0.4237 & 1.4869 & 0.0000 & 1.5461 \\
\hline 0.1341 & 0.0833 & 0.2127 & 8.7140 & 8.2799 & 10.1861 & 8.7140 & -0.4341 & 1.4721 & 0.0000 & 1.5348 \\
\hline 0.1341 & 0.0878 & 0.2134 & 9.5909 & 9.1233 & 11.0949 & 9.5909 & -0.4676 & 1.5040 & 0.0000 & 1.5750 \\
\hline 0.1453 & 0.0908 & 0.2127 & 8.8062 & 8.4015 & 10.1656 & 8.8062 & -0.4048 & 1.3593 & 0.0000 & 1.4183 \\
\hline 0.1521 & 0.0938 & 0.2131 & 8.5996 & 8.2193 & 9.8910 & 8.5996 & -0.3804 & 1.2914 & 0.0000 & 1.3462 \\
\hline 0.1528 & 0.0968 & 0.2142 & 9.0298 & 8.6369 & 10.3286 & 9.0299 & -0.3930 & 1.2988 & 0.0000 & 1.3569 \\
\hline 0.1558 & 0.0998 & 0.2142 & 9.2140 & 8.8222 & 10.4931 & 9.2140 & -0.3917 & 1.2792 & 0.0000 & 1.3378 \\
\hline 0.1577 & 0.1020 & 0.2134 & 9.3885 & 8.9956 & 10.6576 & 9.3885 & -0.3929 & 1.2691 & 0.0000 & 1.3285 \\
\hline 0.1663 & 0.1043 & 0.2164 & 8.8660 & 8.5091 & 10.0531 & 8.8660 & -0.3569 & 1.1870 & 0.0000 & 1.2395 \\
\hline 0.1667 & 0.1057 & 0.2119 & 9.0631 & 8.7009 & 10.2532 & 9.0631 & -0.3622 & 1.1901 & 0.0000 & 1.2440 \\
\hline 0.1731 & 0.1065 & 0.2116 & 8.5701 & 8.2356 & 9.7018 & 8.5701 & -0.3345 & 1.1317 & 0.0000 & 1.1801 \\
\hline 0.1686 & 0.1072 & 0.2108 & 9.1111 & 8.7514 & 10.2892 & 9.1111 & -0.3597 & 1.1781 & 0.0000 & 1.2318 \\
\hline 0.1712 & 0.1072 & 0.2131 & 8.8575 & 8.5108 & 10.0100 & 8.8575 & -0.3467 & 1.1525 & 0.0000 & 1.2035 \\
\hline 0.1701 & 0.1080 & 0.2123 & 9.0798 & 8.7241 & 10.2465 & 9.0798 & -0.3557 & 1.1667 & 0.0000 & 1.2197 \\
\hline 0.1678 & 0.1087 & 0.2119 & 9.4198 & 9.0490 & 10.6122 & 9.4198 & -0.3708 & 1.1924 & 0.0000 & 1.2487 \\
\hline 0.1716 & 0.1080 & 0.2131 & 8.9354 & 8.5871 & 10.0876 & 8.9354 & -0.3483 & 1.1522 & 0.0000 & 1.2037 \\
\hline 0.1682 & 0.1087 & 0.2127 & 9.3820 & 9.0132 & 10.5706 & 9.3820 & -0.3688 & 1.1886 & 0.0000 & 1.2445 \\
\hline 0.1701 & 0.1080 & 0.2149 & 9.0799 & 8.7243 & 10.2466 & 9.0800 & -0.3557 & 1.1667 & 0.0000 & 1.2197 \\
\hline 0.1727 & 0.1087 & 0.2161 & 8.9417 & 8.5954 & 10.0865 & 8.9417 & -0.3463 & 1.1448 & 0.0000 & 1.1960 \\
\hline 0.1675 & 0.1080 & 0.2123 & 9.3400 & 8.9708 & 10.5328 & 9.3400 & -0.3691 & 1.1928 & 0.0000 & 1.2486 \\
\hline 0.1701 & 0.1087 & 0.2134 & 9.1951 & 8.8359 & 10.3651 & 9.1951 & -0.3592 & 1.1700 & 0.0000 & 1.2239 \\
\hline 0.1738 & 0.1095 & 0.2146 & 8.9475 & 8.6032 & 10.0850 & 8.9475 & -0.3443 & 1.1375 & 0.0000 & 1.1884 \\
\hline 0.1742 & 0.1087 & 0.2138 & 8.8009 & 8.4617 & 9.9317 & 8.8009 & -0.3392 & 1.1308 & 0.0000 & 1.1806 \\
\hline 0.1701 & 0.1087 & 0.2127 & 9.1947 & 8.8355 & 10.3647 & 9.1947 & -0.3592 & 1.1700 & 0.0000 & 1.2239 \\
\hline 0.1674 & 0.1087 & 0.2157 & 9.4574 & 9.0846 & 10.6536 & 9.4574 & -0.3728 & 1.1962 & 0.0000 & 1.2530 \\
\hline 0.1701 & 0.1095 & 0.2146 & 9.3102 & 8.9475 & 10.4835 & 9.3102 & -0.3627 & 1.1733 & 0.0000 & 1.2281 \\
\hline 0.1716 & 0.1087 & 0.2145 & 9.0480 & 8.6962 & 10.2035 & 9.0480 & -0.3517 & 1.1555 & 0.0000 & 1.2079 \\
\hline 0.1723 & 0.1087 & 0.2130 & 8.9765 & 8.6284 & 10.1248 & 8.9765 & -0.3481 & 1.1484 & 0.0000 & 1.1999 \\
\hline 0.1738 & 0.1095 & 0.2172 & 8.9466 & 8.6023 & 10.0841 & 8.9466 & -0.3443 & 1.1375 & 0.0000 & 1.1885 \\
\hline 0.1701 & 0.1087 & 0.2149 & 9.1938 & 8.8346 & 10.3638 & 9.1938 & -0.3592 & 1.1700 & 0.0000 & 1.2239 \\
\hline 0.1637 & 0.1087 & 0.2123 & 9.8497 & 9.4562 & 11.0854 & 9.8497 & -0.3935 & 1.2357 & 0.0000 & 1.2969 \\
\hline 0.1708 & 0.1087 & 0.2111 & 9.1194 & 8.7639 & 10.2822 & 9.1194 & -0.3554 & 1.1628 & 0.0000 & 1.2159 \\
\hline 0.1727 & 0.1087 & 0.2119 & 8.9395 & 8.5932 & 10.0844 & 8.9395 & -0.3463 & 1.1449 & 0.0000 & 1.1961 \\
\hline 0.1671 & 0.1087 & 0.2115 & 9.4949 & 9.1201 & 10.6950 & 9.4949 & -0.3748 & 1.2001 & 0.0000 & 1.2573 \\
\hline 0.1671 & 0.1087 & 0.2134 & 9.4952 & 9.1204 & 10.6953 & 9.4952 & -0.3748 & 1.2001 & 0.0000 & 1.2573 \\
\hline 0.1671 & 0.1087 & 0.2142 & 9.4958 & 9.1210 & 10.6959 & 9.4958 & -0.3748 & 1.2001 & 0.0000 & 1.2572 \\
\hline 0.1674 & 0.1087 & 0.2119 & 9.4562 & 9.0834 & 10.6525 & 9.4562 & -0.3728 & 1.1963 & 0.0000 & 1.2530 \\
\hline 0.1704 & 0.1102 & 0.2123 & 9.3872 & 9.0229 & 10.5602 & 9.3872 & -0.3643 & 1.1730 & 0.0000 & 1.2282 \\
\hline 0.1693 & 0.1095 & 0.2134 & 9.3840 & 9.0175 & 10.5648 & 9.3840 & -0.3665 & 1.1808 & 0.0000 & 1.2363 \\
\hline 0.1727 & 0.1087 & 0.2123 & 8.9396 & 8.5933 & 10.0845 & 8.9396 & -0.3463 & 1.1449 & 0.0000 & 1.1961 \\
\hline 0.1671 & 0.1095 & 0.2123 & 9.6125 & 9.2340 & 10.8161 & 9.6125 & -0.3784 & 1.2036 & 0.0000 & 1.2617 \\
\hline 0.1712 & 0.1102 & 0.2126 & 9.3113 & 8.9508 & 10.4771 & 9.3113 & -0.3605 & 1.1657 & 0.0000 & 1.2202 \\
\hline 0.1678 & 0.1102 & 0.2130 & 9.6523 & 9.2743 & 10.8517 & 9.6523 & -0.3780 & 1.1994 & 0.0000 & 1.2576 \\
\hline 0.1712 & 0.1095 & 0.2138 & 9.1965 & 8.8395 & 10.3590 & 9.1965 & -0.3570 & 1.1625 & 0.0000 & 1.2161 \\
\hline 0.1745 & 0.1094 & 0.2149 & 8.8742 & 8.5335 & 10.0049 & 8.8743 & -0.3408 & 1.1307 & 0.0000 & 1.1809 \\
\hline 0.1685 & 0.1102 & 0.2141 & 9.5754 & 9.2013 & 10.7671 & 9.5754 & -0.3740 & 1.1917 & 0.0000 & 1.2491 \\
\hline 0.1674 & 0.1087 & 0.2126 & 9.4547 & 9.0819 & 10.6511 & 9.4547 & -0.3728 & 1.1964 & 0.0000 & 1.2531 \\
\hline 0.1693 & 0.1094 & 0.2122 & 9.3819 & 9.0154 & 10.5628 & 9.3819 & -0.3665 & 1.1809 & 0.0000 & 1.2365 \\
\hline 0.1715 & 0.1087 & 0.2122 & 9.0454 & 8.6937 & 10.2011 & 9.0454 & -0.3517 & 1.1556 & 0.0000 & 1.2080 \\
\hline
\end{tabular}


The calculated uncertainties for $\beta$ show that the overall uncertainty appears to be about $\pm 1.25^{\circ}$. Given the behavior of the uncertainty in the wind tunnel environment, the uncertainty of the measurements in a flight test environment can be expected to be better due flight dynamic pressures being three to five times that of the wind tunnel, thereby utilizing more of the pressure sensor range and decreasing the relative uncertainty in measured quantities. Since the uncertainty obtained scales linearly with perturbed uncertainty values, the expected uncertainty for $\beta$ in flight tests would be decreased by a factor of three to five. An estimate of the uncertainty with respect to flight tests would be $\pm 0.2^{\circ}$ to $\pm 0.5^{\circ}$.

\section{Table D.2 $-\frac{q}{q_{R E F}}$ uncertainty results from flat plate data}

\begin{tabular}{|c|c|c|c|c|c|c|c|c|c|c|}
\hline$x 1\left(P_{L}\right)$ & $x 2\left(P_{L-R}\right)$ & x3 (q $\left.q_{\text {REF }}\right)$ & q|qe & $q \mid q e\left(x_{1}+u_{x 1}\right)$ & $q \mid q e\left(x_{1}+u_{x_{1}}\right)$ & $q \mid q e\left(x_{1}+u_{x_{1}}\right)$ & $\Delta \mathrm{x} 1$ & $\Delta \times 2$ & $\Delta \times 3$ & $u_{q \mid q e}$ \\
\hline 0.0730 & 0.0495 & 0.2120 & 0.3957 & 0.4250 & 0.3702 & 0.3888 & 0.0293 & -0.0255 & -0.0069 & 0.0394 \\
\hline 0.0767 & 0.0495 & 0.2127 & 0.4235 & 0.4529 & 0.3976 & 0.4162 & 0.0294 & -0.0259 & -0.0073 & 0.0399 \\
\hline 0.0808 & 0.0495 & 0.2119 & 0.4575 & 0.4871 & 0.4308 & 0.4495 & 0.0297 & -0.0266 & -0.0080 & 0.0406 \\
\hline 0.0778 & 0.0495 & 0.2116 & 0.4346 & 0.4642 & 0.4084 & 0.4270 & 0.0296 & -0.0262 & -0.0076 & 0.0403 \\
\hline 0.0876 & 0.0540 & 0.2153 & 0.4866 & 0.5158 & 0.4604 & 0.4783 & 0.0292 & -0.0262 & -0.0083 & 0.0401 \\
\hline 0.1067 & 0.0593 & 0.2127 & 0.6243 & 0.6541 & 0.5967 & 0.6135 & 0.0298 & -0.0276 & -0.0108 & 0.0421 \\
\hline 0.1101 & 0.0645 & 0.2120 & 0.6337 & 0.6635 & 0.6065 & 0.6227 & 0.0298 & -0.0272 & -0.0110 & 0.0418 \\
\hline 0.1153 & 0.0698 & 0.2142 & 0.6490 & 0.6784 & 0.6223 & 0.6379 & 0.0294 & -0.0267 & -0.0112 & 0.0412 \\
\hline 0.1311 & 0.0788 & 0.2138 & 0.7409 & 0.7703 & 0.7140 & 0.7281 & 0.0294 & -0.0269 & -0.0128 & 0.0418 \\
\hline 0.1341 & 0.0833 & 0.2127 & 0.7520 & 0.7815 & 0.7253 & 0.7390 & 0.0295 & -0.0267 & -0.0130 & 0.0419 \\
\hline 0.1341 & 0.0878 & 0.2134 & 0.7333 & 0.7625 & 0.7073 & 0.7206 & 0.0292 & -0.0260 & -0.0127 & 0.0411 \\
\hline 0.1453 & 0.0908 & 0.2127 & 0.8132 & 0.8427 & 0.7866 & 0.7991 & 0.0295 & -0.0267 & -0.0141 & 0.0422 \\
\hline 0.1521 & 0.0938 & 0.2131 & 0.8539 & 0.8834 & 0.8271 & 0.8391 & 0.0295 & -0.0268 & -0.0148 & 0.0425 \\
\hline 0.1528 & 0.0968 & 0.2142 & 0.8445 & 0.8737 & 0.8181 & 0.8300 & 0.0292 & -0.0264 & -0.0145 & 0.0420 \\
\hline 0.1558 & 0.0998 & 0.2142 & 0.8572 & 0.8864 & 0.8309 & 0.8424 & 0.0292 & -0.0263 & -0.0147 & 0.0419 \\
\hline 0.1577 & 0.1020 & 0.2134 & 0.8668 & 0.8961 & 0.8406 & 0.8518 & 0.0293 & -0.0262 & -0.0150 & 0.0421 \\
\hline 0.1663 & 0.1043 & 0.2164 & 0.9133 & 0.9422 & 0.8870 & 0.8977 & 0.0290 & -0.0262 & -0.0156 & 0.0420 \\
\hline 0.1731 & 0.1065 & 0.2116 & 0.9794 & 1.0091 & 0.9524 & 0.9624 & 0.0297 & -0.0270 & -0.0171 & 0.0436 \\
\hline 0.1686 & 0.1072 & 0.2108 & 0.9445 & 0.9742 & 0.9178 & 0.9280 & 0.0297 & -0.0268 & -0.0165 & 0.0432 \\
\hline 0.1712 & 0.1072 & 0.2131 & 0.9551 & 0.9845 & 0.9285 & 0.9386 & 0.0294 & -0.0266 & -0.0165 & 0.0430 \\
\hline 0.1701 & 0.1080 & 0.2123 & 0.9470 & 0.9764 & 0.9204 & 0.9305 & 0.0295 & -0.0266 & -0.0164 & 0.0430 \\
\hline 0.1678 & 0.1087 & 0.2119 & 0.9283 & 0.9577 & 0.9018 & 0.9121 & 0.0295 & -0.0264 & -0.0161 & 0.0427 \\
\hline 0.1716 & 0.1080 & 0.2131 & 0.9554 & 0.9847 & 0.9288 & 0.9388 & 0.0294 & -0.0266 & -0.0165 & 0.0430 \\
\hline 0.1682 & 0.1087 & 0.2127 & 0.9279 & 0.9573 & 0.9016 & 0.9118 & 0.0294 & -0.0264 & -0.0161 & 0.0426 \\
\hline 0.1701 & 0.1080 & 0.2149 & 0.9354 & 0.9645 & 0.9091 & 0.9193 & 0.0291 & -0.0263 & -0.0160 & 0.0424 \\
\hline 0.1727 & 0.1087 & 0.2161 & 0.9481 & 0.9771 & 0.9219 & 0.9319 & 0.0290 & -0.0262 & -0.0162 & 0.0423 \\
\hline 0.1675 & 0.1080 & 0.2123 & 0.9264 & 0.9558 & 0.8999 & 0.9103 & 0.0294 & -0.0264 & -0.0161 & 0.0427 \\
\hline 0.1701 & 0.1087 & 0.2134 & 0.9393 & 0.9686 & 0.9129 & 0.9231 & 0.0293 & -0.0264 & -0.0162 & 0.0426 \\
\hline 0.1738 & 0.1095 & 0.2146 & 0.9608 & 0.9900 & 0.9344 & 0.9443 & 0.0292 & -0.0264 & -0.0165 & 0.0427 \\
\hline 0.1742 & 0.1087 & 0.2138 & 0.9698 & 0.9991 & 0.9432 & 0.9531 & 0.0293 & -0.0266 & -0.0167 & 0.0430 \\
\hline 0.1701 & 0.1087 & 0.2127 & 0.9426 & 0.9720 & 0.9161 & 0.9263 & 0.0294 & -0.0265 & -0.0163 & 0.0428 \\
\hline
\end{tabular}




\begin{tabular}{|c|c|c|c|c|c|c|c|c|c|c|}
\hline 0.1674 & 0.1087 & 0.2157 & 0.9092 & 0.9382 & 0.8833 & 0.8937 & 0.0289 & -0.0259 & -0.0155 & 0.0419 \\
\hline 0.1701 & 0.1095 & 0.2146 & 0.9317 & 0.9608 & 0.9055 & 0.9157 & 0.0291 & -0.0262 & -0.0160 & 0.0423 \\
\hline 0.1716 & 0.1087 & 0.2145 & 0.9460 & 0.9752 & 0.9197 & 0.9298 & 0.0292 & -0.0263 & -0.0163 & 0.0425 \\
\hline 0.1723 & 0.1087 & 0.2130 & 0.9586 & 0.9880 & 0.9320 & 0.9420 & 0.0294 & -0.0266 & -0.0166 & 0.0430 \\
\hline 0.1738 & 0.1095 & 0.2172 & 0.9492 & 0.9781 & 0.9231 & 0.9331 & 0.0288 & -0.0261 & -0.0161 & 0.0421 \\
\hline 0.1701 & 0.1087 & 0.2149 & 0.9328 & 0.9619 & 0.9066 & 0.9168 & 0.0291 & -0.0262 & -0.0160 & 0.0423 \\
\hline 0.1637 & 0.1087 & 0.2123 & 0.8944 & 0.9237 & 0.8683 & 0.8789 & 0.0293 & -0.0261 & -0.0155 & 0.0422 \\
\hline 0.1708 & 0.1087 & 0.2111 & 0.9553 & 0.9849 & 0.9286 & 0.9386 & 0.0296 & -0.0267 & -0.0167 & 0.0432 \\
\hline 0.1727 & 0.1087 & 0.2119 & 0.9666 & 0.9962 & 0.9399 & 0.9498 & 0.0296 & -0.0267 & -0.0168 & 0.0433 \\
\hline 0.1671 & 0.1087 & 0.2134 & 0.9159 & 0.9452 & 0.8897 & 0.9001 & 0.0292 & -0.0262 & -0.0158 & 0.0423 \\
\hline 0.1671 & 0.1087 & 0.2142 & 0.9127 & 0.9418 & 0.8866 & 0.8970 & 0.0291 & -0.0261 & -0.0157 & 0.0422 \\
\hline 0.1674 & 0.1087 & 0.2119 & 0.9254 & 0.9548 & 0.8990 & 0.9093 & 0.0295 & -0.0264 & -0.0161 & 0.0427 \\
\hline 0.1704 & 0.1102 & 0.2123 & 0.9419 & 0.9713 & 0.9155 & 0.9255 & 0.0294 & -0.0264 & -0.0163 & 0.0428 \\
\hline 0.1693 & 0.1095 & 0.2134 & 0.9308 & 0.9601 & 0.9045 & 0.9147 & 0.0293 & -0.0263 & -0.0161 & 0.0425 \\
\hline 0.1727 & 0.1087 & 0.2123 & 0.9649 & 0.9944 & 0.9382 & 0.9482 & 0.0295 & -0.0267 & -0.0168 & 0.0432 \\
\hline 0.1671 & 0.1095 & 0.2123 & 0.9182 & 0.9475 & 0.8919 & 0.9022 & 0.0294 & -0.0263 & -0.0159 & 0.0425 \\
\hline 0.1712 & 0.1102 & 0.2126 & 0.9461 & 0.9755 & 0.9197 & 0.9297 & 0.0294 & -0.0264 & -0.0164 & 0.0428 \\
\hline 0.1745 & 0.1094 & 0.2149 & 0.9651 & 0.9942 & 0.9387 & 0.9485 & 0.0292 & -0.0264 & -0.0166 & 0.0427 \\
\hline 0.1685 & 0.1102 & 0.2141 & 0.9191 & 0.9483 & 0.8931 & 0.9033 & 0.0291 & -0.0261 & -0.0158 & 0.0422 \\
\hline 0.1674 & 0.1087 & 0.2126 & 0.9222 & 0.9515 & 0.8958 & 0.9062 & 0.0294 & -0.0263 & -0.0160 & 0.0425 \\
\hline 0.1693 & 0.1094 & 0.2122 & 0.9358 & 0.9652 & 0.9094 & 0.9196 & 0.0294 & -0.0264 & -0.0162 & 0.0428 \\
\hline 0.1715 & 0.1087 & 0.2122 & 0.9561 & 0.9856 & 0.9295 & 0.9395 & 0.0295 & -0.0266 & -0.0166 & 0.0431 \\
\hline
\end{tabular}

The calculated uncertainties for $\frac{q}{q_{R E F}}$ show that the overall uncertainty appears to be about \pm 0.04 with $\frac{q}{q_{R E F}}$ varying from 0.4 to nearly 1.0 . Once again, given the behavior of the uncertainty in the wind tunnel environment, the uncertainty of the measurements in a flight test environment can be expected to be better due flight dynamic pressures being three to five times that of the wind tunnel, thereby utilizing more of the pressure sensor range and decreasing relative uncertainty. The uncertainty obtained scales linearly with perturbed uncertainty values, the expected uncertainty for $\frac{q}{q_{R E F}}$ in flight tests would be decreased by a factor of three to five. An estimate of the uncertainty with respect to flight tests would decrease the uncertainty by a factor of about four to about 0.01 . Due to the fact that most boundary layer results are presented in terms of non-dimensional velocity, as opposed to non-dimensional dynamic pressure, the uncertainty results were converted to velocities by taking the square root. This 
yielded uncertainties of \pm 0.02 for $\frac{u}{u_{R E F}}$ in wind tunnel conditions, and \pm 0.01 for $\frac{u}{u_{R E F}}$ in flight test conditions. 